\title{
General structure of the neutral $\rho$ meson self-energy and its spectral properties in a hot and dense magnetized medium
}

\author{
Snigdha Ghosh,,$^{1,2, *}$ Arghya Mukherjee, ${ }^{2,4, \dagger}$ Pradip Roy, ${ }^{2,4, \$}$ and Sourav Sarkar ${ }^{3,4, \S}$ \\ ${ }^{1}$ Indian Institute of Technology Gandhinagar, Palaj, Gandhinagar 382355, Gujarat, India \\ ${ }^{2}$ Saha Institute of Nuclear Physics, 1/AF Bidhannagar, Kolkata 700064, India \\ ${ }^{3}$ Variable Energy Cyclotron Centre, 1/AF Bidhannagar, Kolkata 700064, India \\ ${ }^{4}$ Homi Bhabha National Institute, Training School Complex, Anushaktinagar, Mumbai 400085, India
}

(Received 9 January 2019; published 7 May 2019)

\begin{abstract}
The one-loop self-energy of the neutral $\rho$ meson is obtained for the effective $\rho \pi \pi$ and $\rho N N$ interaction at finite temperature and density in the presence of a constant background magnetic field of arbitrary strength. In our approach, the $e B$-dependent vacuum part of the self-energy is extracted by means of dimensional regularization where the ultraviolet divergences corresponding to the pure-vacuum self-energy manifest as the pole singularities of gamma as well as Hurwitz zeta functions. This improved regularization procedure consistently reproduces the expected results in the vanishing magnetic field limit and can be used quite generally in other self-energy calculations dealing with arbitrary magnetic field strength. In the presence of the external magnetic field, the general Lorentz structure for the in-medium vector boson self-energy is derived, which can also be implemented in the case of the gauge bosons such as photons and gluons. It has been shown that with vanishing perpendicular momentum of the external particle essentially two form factors are sufficient to describe the self-energy completely. Consequently, two distinct modes are observed in the study of the effective mass, dispersion relations and the spectral function of $\rho^{0}$ where one of the modes possesses twofold degeneracy. For large baryonic chemical potential, it is observed that the critical magnetic field required to block the $\rho^{0} \rightarrow \pi^{+} \pi^{-}$decay channel increases significantly with temperature. However, in the case of smaller values reaching down to vanishing chemical potential, the critical field follows the opposite trend.
\end{abstract}

DOI: 10.1103/PhysRevD.99.096004

\section{INTRODUCTION}

In noncentral heavy-ion collisions (HIC) at the LHC, the relative motion of the ions themselves can generate a strong decaying magnetic pulse of the order $e B \sim 15 m_{\pi}^{2}$ $\left(B \sim 5 \times 10^{15} \mathrm{~T}\right)$ [1]. While some of the studies support a rapid decrease in the magnitude [2,3], an adiabatic decay is expected [4-6] due to the high conductivity of the produced medium. In spite of the ambiguities, the intensity of the produced magnetic field being much larger than the typical QCD scale, the possibility of magnetic modifications of different properties of the produced extreme state of matter can not be refuted completely. In general, high

\footnotetext{
*snigdha.physics@gmail.com, snigdha.ghosh@saha.ac.in †arghya.mukherjee@saha.ac.in

pradipk.roy@saha.ac.in

ssourav@vecc.gov.in
}

Published by the American Physical Society under the terms of the Creative Commons Attribution 4.0 International license. Further distribution of this work must maintain attribution to the author(s) and the published article's title, journal citation, and DOI. Funded by SCOAP ${ }^{3}$. intensity magnetic fields can play a significant role in many astrophysical and cosmological phenomena [7-10]. Moreover, the magnetic influence on the properties of magnetars adds to the motivation of studying high density matter in the presence of extreme magnetic fields [11-18].

The study of $\rho$ meson properties like the effective mass and dispersion relations are important in the context of magnetic field induced vacuum superconductivity [19-26]. Using the Nambu-Jona-Lasinio model in the presence of magnetic background, Liu et al. have shown that the charged $\rho$ condensation in vacuum occurs at critical magnetic field $e B_{c} \sim 0.2 \mathrm{GeV}^{2}$ [25]. Generalization of the study to finite temperature and density shows that the condensation survives even in the presence of finite temperature and density [27]. At vanishing chemical potential, the corresponding critical magnetic field is observed to lie in the range $0.2-0.6 \mathrm{GeV}^{2}$ for temperatures in between 0.2 and $0.5 \mathrm{GeV}$. However, the neutral $\rho$ meson in vacuum, having no trivial Landau shifts in the energy eigenvalue, shows a slow decrease in the effective mass [28] in the weak magnetic field region. Thus, if neutral $\rho$ condensation is possible, extremely large magnetic field values will be required to observe the condensation. 
It should be mentioned here that it has been shown using the Nambu-Jona-Lasinio model that the effective mass of a $\rho^{0}$ meson in fact increases at higher values of magnetic fields, showing no possibility of condensation [25]. In this scenario, $\rho^{0} \rightarrow \pi^{+} \pi^{-}$decay may serve as an important probe to observe the influence of the magnetic field. As argued in Ref. [19], even if a pointlike $\rho^{0}$ meson is considered without any influence by magnetic field, there exists a critical value of the external magnetic field for which the $\rho^{0}$ to $\pi^{+} \pi^{-}$decay stops due to the trivial enhancement of the charged pion mass. Later, the magnetic modification arising from the loop corrections is taken into account at weak [28,29] as well as at strong field limits [30] at zero temperature. An immediate generalization of the previous works will be to incorporate the medium effects of the $\rho^{0}$ meson, which may reflect in the modification of the decay rate and the required critical magnetic field. It should be noted here that, apart from being important in the study of dense hadronic matter at extreme conditions usually expected to be present within compact stars, the incorporation of the medium effects is also essential for the proper estimation of pion production in noncentral heavy-ion collisions.

In this work, we focus on the temperature and density modifications of neutral $\rho$ meson properties in the presence of a static homogeneous magnetic background. The oneloop self-energy of a $\rho$ meson is calculated for the effective $\rho \pi \pi$ and $\rho N N$ interaction with magnetically modified pion and nucleon propagators corresponding to general field strength. After decomposing the self-energy in terms of the form factors, the decay width for the $\rho^{0} \rightarrow \pi^{+} \pi^{-}$channel is obtained. It should be mentioned here that the spectral properties of the $\rho$ meson in the presence of finite temperature and magnetic field were studied in our earlier work [31]. However, unlike the previous case, the dimensional regularization technique is used here to extract the ultraviolet divergence as pole singularities of gamma and Hurwitz zeta functions [32]. Also, instead of considering only the spin-averaged thermal self-energy contribution, the general Lorentz structure has been addressed in detail.

Apart from the technical differences, the density dependence arising from the charged nucleon loop serves as the most important extension of the previous study. Its importance can be understood as follows. It is well known that the general expression of decay width is related to the imaginary part of the self-energy. Now, as far as the $\rho^{0} \rightarrow$ $\pi^{+} \pi^{-}$decay is concerned, the invariant mass regime of interest does not allow the nucleon loop to directly contribute to the imaginary part as the unitary cut threshold of the $N N$ loop begins at a much higher value. However, it should be noted that in the rest frame of the decaying particle the decay width depends on its effective mass. The contribution from the nucleon loop incorporates significant modification in the effective mass of $\rho^{0}$, which in turn influences the decay. As we shall see, the critical field required to stabilize the neutral $\rho$ against the $\pi^{+} \pi^{-}$decay has a nontrivial dependence on the baryonic chemical potential.

The article is organized as follows. In Sec. II, the vacuum self-energy of $\rho$ is discussed, followed by evaluation of the in-medium $\rho$ self-energy at zero magnetic field in Sec. III. Next, in Sec. IV, the in-medium self-energy at nonzero external magnetic field is presented. Section V is devoted to the discussion of the general Lorentz structure of the inmedium self-energy function in the presence of a constant background magnetic field. After addressing the Lorentz structure of the interacting $\rho$ propagator in Sec. VI, the analytic structure of the self-energy is discussed in Sec. VII. Section VIII contains the numerical results. Finally, we summarize and conclude in Sec. IX. Some of the relevant calculational details are provided in the Appendix.

\section{II. $\rho^{0}$ SELF-ENERGY IN THE VACUUM}

The effective Lagrangian for $\rho \pi \pi$ and $\rho N N$ interaction is [33]

$$
\begin{aligned}
\mathcal{L}_{\text {int }}= & -g_{\rho \pi \pi} \partial_{\mu} \vec{\rho}_{\nu} \cdot\left(\partial^{\mu} \vec{\pi} \times \partial^{\nu} \vec{\pi}\right) \\
& -g_{\rho N N} \bar{\Psi}\left[\gamma^{\mu}-\frac{\kappa_{\rho}}{2 m_{N}} \sigma^{\mu \nu} \partial_{\nu}\right] \vec{\tau} \cdot \vec{\rho}_{\mu} \Psi,
\end{aligned}
$$

where $\Psi=\left[\begin{array}{l}p \\ n\end{array}\right]$ is the nucleon isospin doublet, $\sigma^{\mu \nu}=\frac{i}{2}\left[\gamma^{\mu}, \gamma^{\nu}\right]$, and the components of $\vec{\tau}$ correspond to the Pauli isospin matrices. It is understood that the derivative within the square bracket in the above equation acts only on the $\rho$ field. The value of the coupling constants are given by $g_{\rho \pi \pi}=20.72 \mathrm{GeV}^{-2}, g_{\rho N N}=3.25$, and $\kappa_{\rho}=6.1$ with $m_{N}=939 \mathrm{MeV}$ as the mass of the nucleons. The metric tensor in this work is taken as $g^{\mu \nu}=\operatorname{diag}(1,-1,-1,-1)$. Using Eq. (1), the one-loop vacuum self-energy of $\rho^{0}$ is obtained as

$$
\Pi_{\text {pure-vac }}^{\mu \nu}=\left(\Pi_{\pi}^{\mu \nu}\right)_{\text {pure-vac }}+\left(\Pi_{\mathrm{N}}^{\mu \nu}\right)_{\text {pure-vac }},
$$

where $\left(\Pi_{\pi}^{\mu \nu}\right)_{\text {pure-vac }}$ and $\left(\Pi_{\mathrm{N}}^{\mu \nu}\right)_{\text {pure-vac }}$ are respectively the contributions from the $\pi \pi$ loop and $N N$ loop, which are given by (applying Feynman rules to Fig. 1)

$$
\begin{aligned}
& \left(\Pi_{\pi}^{\mu \nu}\right)_{\text {pure-vac }}(q) \\
& =i \int \frac{d^{4} k}{(2 \pi)^{4}} \mathcal{N}_{\pi}^{\mu \nu}(q, k) \Delta_{F}\left(k, m_{\pi}\right) \Delta_{F}\left(p=q+k, m_{\pi}\right) \\
& \left(\Pi_{\mathrm{N}}^{\mu \nu}\right)_{\text {pure-vac }}(q) \\
& =-i \int \frac{d^{4} k}{(2 \pi)^{4}} \operatorname{Tr}\left[\Gamma^{\nu}(q) S_{\mathrm{p}}\left(p=q+k, m_{N}\right) \Gamma^{\mu}(-q) S_{\mathrm{p}}\left(k, m_{N}\right)\right. \\
& \left.\quad+\Gamma^{\nu}(q) S_{\mathrm{n}}\left(p=q+k, m_{N}\right) \Gamma^{\mu}(-q) S_{\mathrm{n}}\left(k, m_{N}\right)\right],
\end{aligned}
$$

where 


$$
\Delta_{F}\left(k, m_{\pi}\right)=\frac{-1}{k^{2}-m_{\pi}^{2}+i \epsilon}
$$

is the vacuum Feynman propagator for the charged pion. $S_{\mathrm{p}}$ and $S_{\mathrm{n}}$ are respectively the vacuum Feynman propagators for proton and neutron and are given by

$$
S_{\mathrm{p}}\left(k, m_{N}\right)=S_{\mathrm{n}}\left(k, m_{N}\right)=\left(\not k+m_{N}\right) \Delta_{F}\left(k, m_{N}\right) .
$$

The second rank tensor $\mathcal{N}_{\pi}^{\mu \nu}(q, k)$ and the vector $\Gamma^{\mu}(q)$ in Eqs. (3) and (4) contain the factors coming from the interaction vertices:

$$
\begin{aligned}
\mathcal{N}_{\pi}^{\mu \nu}(q, k)= & g_{\rho \pi \pi}^{2}\left[q^{4} k^{\mu} k^{\nu}+(q \cdot k)^{2} q^{\mu} q^{\nu}\right. \\
& \left.-q^{2}(q \cdot k)\left(q^{\mu} k^{\nu}+q^{\nu} k^{\mu}\right)\right] \\
\Gamma^{\mu}(q)= & g_{\rho N N}\left[\gamma^{\mu}-i \frac{\kappa_{\rho}}{2 m_{N}} \sigma^{\mu \nu} q_{\nu}\right] .
\end{aligned}
$$

The evaluations of the momentum integrals in Eqs. (3) and (4) are briefly sketched in Appendix B, and the final results can be read off from Eqs. (B10) and (B11),

$$
\begin{aligned}
\left(\Pi_{\pi}^{\mu \nu}\right)_{\text {pure-vac }}(q) & \\
= & \left(q^{2} g^{\mu \nu}-q^{\mu} q^{\nu}\right)\left(\frac{-g_{\rho \pi \pi}^{2} q^{2}}{32 \pi^{2}}\right) \\
& \times\left.\int_{0}^{1} d x \Delta_{\pi}\left[\frac{1}{\varepsilon}-\gamma_{\mathrm{E}}+1-\ln \left(\frac{\Delta_{\pi}}{4 \pi \Lambda_{\pi}}\right)\right]\right|_{\varepsilon \rightarrow 0} \\
\left(\Pi_{\mathrm{N}}^{\mu \nu}\right)_{\text {pure-vac }}(q) & \\
= & \left(q^{2} g^{\mu \nu}-q^{\mu} q^{\nu}\right)\left(\frac{g_{\rho N N}^{2}}{2 \pi^{2}}\right) \\
& \times \int_{0}^{1} d x\left[\left\{2 x(1-x)+\kappa_{\rho}+\frac{\kappa_{\rho}^{2}}{2}-\frac{\kappa_{\rho}^{2}}{4 m_{N}^{2}} \Delta_{\mathrm{N}}\right\}\right. \\
& \left.\times\left\{\frac{1}{\varepsilon}-\gamma_{\mathrm{E}}-\ln \left(\frac{\Delta_{\mathrm{N}}}{4 \pi \Lambda_{\mathrm{N}}}\right)\right\}-\frac{\kappa_{\rho}^{2}}{4 m_{N}^{2}} \Delta_{\mathrm{N}}\right]\left.\right|_{\varepsilon \rightarrow 0},
\end{aligned}
$$

where $\Delta_{\pi}$ and $\Delta_{\mathrm{N}}$ are defined in Eqs. (B6) and (B7). As can be seen from the above equations, the vacuum self-energy is divergent and scale dependent, which renormalizes the bare $\rho^{0}$ mass to its physical mass after adding proper vacuum counterterms in the Lagrangian. The particular Lorentz structure in the above equations renders the self-energy transverse to the $\rho^{0}$ momentum, i.e., $q_{\mu} \Pi_{\text {pure-vac }}^{\mu \nu}=0$.

\section{III. $\rho^{0}$ SELF-ENERGY IN THE MEDIUM}

In order to calculate the $\rho^{0}$ self-energy at finite temperature and density, we employ the real time formalism (RTF) of finite temperature field theory where all the two point correlation functions such as the propagator and the selfenergy become $2 \times 2$ matrices in the thermal space [34,35].
However, they can be put in a diagonal form where the diagonal elements can be obtained from any one component (say the 11-component) of the mentioned $2 \times 2$ matrix. The 11-components of real time thermal pion and nucleon propagators are

$$
D^{11}(k)=\Delta_{F}\left(k, m_{\pi}\right)+\eta(k \cdot u)\left[\Delta_{F}\left(k, m_{\pi}\right)-\Delta_{F}^{*}\left(k, m_{\pi}\right)\right]
$$

$$
\begin{aligned}
S_{\mathrm{p}, \mathrm{n}}^{11}(k)= & S_{\mathrm{p}, \mathrm{n}}\left(k, m_{N}\right) \\
& -\tilde{\eta}(k \cdot u)\left[S_{\mathrm{p}, \mathrm{n}}\left(k, m_{N}\right)-\gamma^{0} S_{\mathrm{p}, \mathrm{n}}^{\dagger}\left(k, m_{N}\right) \gamma^{0}\right],
\end{aligned}
$$

where $\quad \eta(x)=\Theta(x) f(x)+\Theta(-x) f(-x)$ and $\tilde{\eta}(x)=$ $\Theta(x) f^{+}(x)+\Theta(-x) f^{-}(-x)$ in which $f(x)$ and $f^{ \pm}(x)$ are respectively the Bose-Einstein and Fermi-Dirac distribution functions corresponding to pions and nucleons:

$f(x)=\left[e^{x / T}-1\right]^{-1}, \quad f^{ \pm}(x)=\left[e^{\left(x \mp \mu_{B}\right) / T}+1\right]^{-1}$.

Here, $\Theta(x)$ is the unit step function, and $u^{\mu}$ is the medium four-velocity; $T$ and $\mu_{B}$ are respectively the temperature and baryon chemical potential of the medium. In the local rest frame (LRF) of the medium, $u_{\mathrm{LRF}}^{\mu} \equiv(1, \overrightarrow{0})$.

For the evaluation of the 11-component of the thermal self-energy matrix, the vacuum pion and nucleon propagators in Eqs. (3) and (4) are replaced by the respective 11components of the thermal propagators given in Eqs. (11) and (12) as [35]

$$
\begin{aligned}
\left(\Pi_{\pi}^{\mu \nu}\right)_{11}(q)= & i \int \frac{d^{4} k}{(2 \pi)^{4}} \mathcal{N}_{\pi}^{\mu \nu}(q, k) D^{11}\left(k, m_{\pi}\right) \\
& \times D^{11}\left(p=q+k, m_{\pi}\right) \\
\left(\Pi_{\mathrm{N}}^{\mu \nu}\right)_{11}(q)= & -i \int \frac{d^{4} k}{(2 \pi)^{4}} \operatorname{Tr}\left[\Gamma^{\nu}(q) S_{\mathrm{p}}^{11}\left(k, m_{N}\right) \Gamma^{\mu}(-q)\right. \\
& \times S_{\mathrm{p}}^{11}\left(p=q+k, m_{N}\right) \\
& +\Gamma^{\nu}(q) S_{\mathrm{n}}^{11}\left(k, m_{N}\right) \Gamma^{\mu}(-q) \\
& \left.\times S_{\mathrm{n}}^{11}\left(p=q+k, m_{N}\right)\right] .
\end{aligned}
$$

The analytic thermal self-energy function of $\rho^{0}$ denoted by a bar $\operatorname{Re} \bar{\Pi}^{\mu \nu}\left(q^{0}, \vec{q}\right)=\operatorname{Re} \bar{\Pi}_{\pi}^{\mu \nu}\left(q^{0}, \vec{q}\right)+\operatorname{Re} \bar{\Pi}_{\mathrm{N}}^{\mu \nu}\left(q^{0}, \vec{q}\right)$ is related to the above quantities by the relations [35]

$$
\begin{aligned}
& \operatorname{Re} \bar{\Pi}_{\pi, N}^{\mu \nu}\left(q^{0}, \vec{q}\right)=\left(\operatorname{Re} \Pi_{\pi, N}^{\mu \nu}\right)_{11}\left(q^{0}, \vec{q}\right) \\
& \operatorname{Im} \bar{\Pi}_{\pi, N}^{\mu \nu}\left(q^{0}, \vec{q}\right)=\operatorname{sign}\left(q^{0}\right) \tanh \left(\frac{q^{0}}{2 T}\right)\left(\operatorname{Im} \Pi_{\pi, N}^{\mu \nu}\right)_{11}\left(q^{0}, \vec{q}\right)
\end{aligned}
$$

where $\operatorname{sign}(x)=\Theta(x)-\Theta(-x)$. Rewriting Eqs. (11) and (12) as 


$$
\begin{aligned}
& D^{11}(k)=\Delta_{F}\left(k, m_{\pi}\right)+2 \pi i \eta(k \cdot u) \delta\left(k^{2}-m_{\pi}^{2}\right) \\
& S_{\mathrm{p}, \mathrm{n}}^{11}(k)=\left(\not k+m_{N}\right)\left[\Delta_{F}\left(k, m_{N}\right)-2 \pi i \tilde{\eta}(k \cdot u) \delta\left(k^{2}-m_{N}^{2}\right)\right]
\end{aligned}
$$

and substituting into Eqs. (14) and (15) and performing the $d k^{0}$ integration (using the Dirac delta functions) followed by using Eqs. (16) and (17), we get the real parts as

$$
\begin{aligned}
\operatorname{Re} \bar{\Pi}_{\pi}^{\mu \nu}\left(q^{0}, \vec{q}\right) & =\operatorname{Re}\left(\Pi_{\pi}^{\mu \nu}\right)_{\text {pure-vac }}(q)+\int \frac{d^{3} k}{(2 \pi)^{3}} \mathcal{P}\left[\frac{f\left(\omega_{k}\right)}{2 \omega_{k}}\left\{\frac{\mathcal{N}_{\pi}^{\mu \nu}\left(k^{0}=-\omega_{k}\right)}{\left(q^{0}-\omega_{k}\right)^{2}-\left(\omega_{p}\right)^{2}}+\frac{\mathcal{N}_{\pi}^{\mu \nu}\left(k^{0}=\omega_{k}\right)}{\left(q^{0}+\omega_{k}\right)^{2}-\left(\omega_{p}\right)^{2}}\right\}\right. \\
& \left.+\frac{f\left(\omega_{p}\right)}{2 \omega_{p}}\left\{\frac{\mathcal{N}_{\pi}^{\mu \nu}\left(k^{0}=-q^{0}-\omega_{p}\right)}{\left(q^{0}+\omega_{p}\right)^{2}-\left(\omega_{k}\right)^{2}}+\frac{\mathcal{N}_{\pi}^{\mu \nu}\left(k^{0}=-q^{0}+\omega_{p}\right)}{\left(q^{0}-\omega_{p}\right)^{2}-\left(\omega_{k}\right)^{2}}\right\}\right] \\
\operatorname{Re} \bar{\Pi}_{\mathrm{N}}^{\mu \nu}\left(q^{0}, \vec{q}\right)= & \operatorname{Re}\left(\Pi_{\mathrm{N}}^{\mu \nu}\right)_{\text {pure-vac }}(q)-\int \frac{d^{3} k}{(2 \pi)^{3}} \mathcal{P}\left[\frac{1}{2 \Omega_{k}}\left\{\frac{f^{-}\left(\Omega_{k}\right) \mathcal{N}_{\mathrm{N}}^{\mu \nu}\left(k^{0}=-\Omega_{k}\right)}{\left(q^{0}-\Omega_{k}\right)^{2}-\left(\Omega_{p}\right)^{2}}+\frac{f^{+}\left(\Omega_{k}\right) \mathcal{N}_{\mathrm{N}}^{\mu \nu}\left(k^{0}=\Omega_{k}\right)}{\left(q^{0}+\Omega_{k}\right)^{2}-\left(\Omega_{p}\right)^{2}}\right\}\right. \\
+ & \left.\frac{1}{2 \Omega_{p}}\left\{\frac{f^{-}\left(\Omega_{p}\right) \mathcal{N}_{\mathrm{N}}^{\mu \nu}\left(k^{0}=-q^{0}-\Omega_{p}\right)}{\left(q^{0}+\Omega_{p}\right)^{2}-\left(\Omega_{k}\right)^{2}}+\frac{f^{+}\left(\Omega_{p}\right) \mathcal{N}_{\mathrm{N}}^{\mu \nu}\left(k^{0}=-q^{0}+\Omega_{p}\right)}{\left(q^{0}-\Omega_{p}\right)^{2}-\left(\Omega_{k}\right)^{2}}\right\}\right]
\end{aligned}
$$

and the imaginary parts as

$$
\begin{aligned}
\operatorname{Im} \bar{\Pi}_{\pi}^{\mu \nu}\left(q^{0}, \vec{q}\right)= & -\operatorname{sign}\left(q^{0}\right) \tanh \left(\frac{\beta q^{0}}{2}\right) \pi \int \frac{d^{3} k}{(2 \pi)^{3}} \frac{1}{4 \omega_{k} \omega_{p}}\left[\left\{1+f\left(\omega_{k}\right)+f\left(\omega_{p}\right)+2 f\left(\omega_{k}\right) f\left(\omega_{p}\right)\right\}\right. \\
& \left\{\mathcal{N}_{\pi}^{\mu \nu}\left(k^{0}=-\omega_{k}\right) \delta\left(q^{0}-\omega_{k}-\omega_{p}\right)+\mathcal{N}_{\pi}^{\mu \nu}\left(k^{0}=\omega_{k}\right) \delta\left(q^{0}+\omega_{k}+\omega_{p}\right)\right\}+\left\{f\left(\omega_{k}\right)+f\left(\omega_{p}\right)+2 f\left(\omega_{k}\right) f\left(\omega_{p}\right)\right\} \\
& \left.\left\{\mathcal{N}_{\pi}^{\mu \nu}\left(k^{0}=-\omega_{k}\right) \delta\left(q^{0}-\omega_{k}+\omega_{p}\right)+\mathcal{N}_{\pi}^{\mu \nu}\left(k^{0}=\omega_{k}\right) \delta\left(q^{0}+\omega_{k}-\omega_{p}\right)\right\}\right]
\end{aligned}
$$

$$
\begin{aligned}
\operatorname{Im} \bar{\Pi}_{\mathrm{N}}^{\mu \nu}\left(q^{0}, \vec{q}\right)= & -\operatorname{sign}\left(q^{0}\right) \tanh \left(\frac{\beta q^{0}}{2}\right) \pi \int \frac{d^{3} k}{(2 \pi)^{3}} \frac{1}{4 \Omega_{k} \Omega_{p}} \\
& \times\left[\left\{1-f^{-}\left(\Omega_{k}\right)-f^{+}\left(\Omega_{p}\right)+2 f^{-}\left(\Omega_{k}\right) f^{+}\left(\Omega_{p}\right)\right\} \mathcal{N}_{\mathbf{N}}^{\mu \nu}\left(k^{0}=-\Omega_{k}\right) \delta\left(q^{0}-\Omega_{k}-\Omega_{p}\right)\right. \\
& +\left\{1-f^{+}\left(\Omega_{k}\right)-f^{-}\left(\Omega_{p}\right)+2 f^{+}\left(\Omega_{k}\right) f^{-}\left(\Omega_{p}\right)\right\} \mathcal{N}_{\mathrm{N}}^{\mu \nu}\left(k^{0}=\Omega_{k}\right) \delta\left(q^{0}+\Omega_{k}+\Omega_{p}\right) \\
& +\left\{-f^{-}\left(\Omega_{k}\right)-f^{-}\left(\Omega_{p}\right)+2 f^{-}\left(\Omega_{k}\right) f^{-}\left(\Omega_{p}\right)\right\} \mathcal{N}_{\mathbf{N}}^{\mu \nu}\left(k^{0}=-\omega_{k}\right) \delta\left(q^{0}-\Omega_{k}+\Omega_{p}\right) \\
& \left.+\left\{-f^{+}\left(\Omega_{k}\right)-f^{+}\left(\Omega_{p}\right)+2 f^{+}\left(\Omega_{k}\right) f^{+}\left(\Omega_{p}\right)\right\} \mathcal{N}_{\mathbf{N}}^{\mu \nu}\left(k^{0}=\Omega_{k}\right) \delta\left(q^{0}+\Omega_{k}-\Omega_{p}\right)\right],
\end{aligned}
$$

where $\mathcal{P}$ denotes the Cauchy principal value integration, $\omega_{k}=\sqrt{m_{\pi}^{2}+\vec{k}^{2}}, \Omega_{k}=\sqrt{m_{N}^{2}+\vec{k}^{2}}$, and $\mathcal{N}_{\mathrm{N}}(q, k)$ is defined in Eq. (B3).

\section{IV. $\rho^{0}$ SELF-ENERGY IN THE MAGNETIZED MEDIUM}

In the presence of the external magnetic field $\vec{B}=B \hat{z}$, the propagations of the charged pion and proton are modified. One of the possible ways to incorporate the effect of external magnetic field is the Schwinger proper time formalism in which the 11-components of charged pion and proton propagators respectively become [36,37]

$$
D_{B}^{11}(k)=\Delta_{B}\left(k, m_{\pi}\right)+\eta(k \cdot u)\left[\Delta_{B}\left(k, m_{\pi}\right)-\Delta_{B}^{*}\left(k, m_{\pi}\right)\right]
$$

and

$$
S_{B}^{11}(k)=S_{B}\left(k, m_{N}\right)-\tilde{\eta}(k \cdot u)\left[S_{B}\left(k, m_{N}\right)-\gamma^{0} S_{B}^{\dagger}\left(k, m_{N}\right) \gamma^{0}\right],
$$

where $\Delta_{B}\left(k, m_{\pi}\right)$ and $S_{B}\left(k, m_{N}\right)$ denote the momentum space vacuum (zero temperature) Schwinger proper time propagators for the charged pion and proton respectively [36]:

$$
\begin{aligned}
\Delta_{B}(k)= & i \int_{0}^{\infty} d s \exp \left[i s\left\{k_{\|}^{2}+\frac{\tan (e B s)}{e B s} k_{\perp}^{2}-m_{N}^{2}\right\}\right] \\
S_{B}(k)= & i \int_{0}^{\infty} d s \exp \left[i s\left\{k_{\|}^{2}+\frac{\tan (e B s)}{e B s} k_{\perp}^{2}-m_{N}^{2}\right\}\right] \\
& \times\left[\left(\not k_{\|}+m_{N}\right)\left\{1-\gamma^{1} \gamma^{2} \tan (e B s)\right\}+\not k_{\perp} \sec ^{2}(e B s)\right] .
\end{aligned}
$$


In the above equations, $e=|e|$ is the charge of the proton; the four-vector $k$ is decomposed into $k=\left(k_{\|}+k_{\perp}\right)$ where $k_{\|}^{\mu}=g_{\|}^{\mu \nu} k_{\nu}$ and $k_{\perp}^{\mu}=g_{\perp}^{\mu \nu} k_{\nu}$, corresponding to the decomposition of the metric tensor $g^{\mu \nu}=\left(g_{\|}^{\mu \nu}+g_{\perp}^{\mu \nu}\right)$ with $g_{\|}^{\mu \nu}=$ $\operatorname{diag}(1,0,0,-1)$ and $g_{\perp}^{\mu \nu}=\operatorname{diag}(0,-1,-1,0)$. The above decomposition can be done in a Lorentz covariant way by introducing another four-vector,

$$
b^{\mu}=\frac{1}{B} G^{\mu \nu} u_{\nu},
$$

where $G^{\mu \nu}=\frac{1}{2} \epsilon^{\mu \nu \alpha \beta} F_{\alpha \beta}$ is the dual of the electromagnetic field tensor $F^{\mu \nu}$. In the local rest frame of the medium, $b_{\mathrm{LRF}}^{\mu} \equiv(0,0,0,1)$, which is the direction of the external magnetic field. Using $b^{\mu}$, we can write

$$
g_{\|}^{\mu \nu}=\left(u^{\mu} u^{\nu}-b^{\mu} b^{\nu}\right) \quad \text { and } \quad g_{\perp}^{\mu \nu}=\left(g^{\mu \nu}-u^{\mu} u^{\nu}+b^{\mu} b^{\nu}\right) \text {. }
$$

It is important to note that the coordinate space Schwinger propagator contains a gauge-dependent translationally noninvariant phase factor. However, for the oneloop graphs containing equally charged particles in the loop, the phase factor gets canceled, and the momentum space propagator is sufficient for the calculation of the selfenergy. The proper time integral in Eqs. (26) and (27) can be performed in order to express the propagators as a sum over discrete Landau levels as

$$
\begin{gathered}
\Delta_{B}(k)=-\sum_{l=0}^{\infty} \frac{2(-1)^{l} e^{-\alpha_{k}} L_{l}\left(2 \alpha_{k}\right)}{k_{\|}^{2}-m_{\pi}^{2}-(2 l+1) e B+i \epsilon} \\
S_{B}(k)=-\sum_{l=0}^{\infty}\left[\frac{(-1)^{l} e^{-\alpha_{k}} \mathcal{D}_{l}(k)}{k_{\|}^{2}-m_{N}^{2}-2 l e B+i \epsilon}\right],
\end{gathered}
$$

where

$$
\begin{aligned}
\mathcal{D}_{l}(k)= & \left(\not \|_{\|}+m_{N}\right)\left[\left(1+i \gamma^{1} \gamma^{2}\right) L_{l}\left(2 \alpha_{k}\right)\right. \\
& \left.-\left(1-i \gamma^{1} \gamma^{2}\right) L_{l-1}\left(2 \alpha_{k}\right)\right]-4 \not k_{\perp} L_{l-1}^{1}\left(2 \alpha_{k}\right)
\end{aligned}
$$

with $\alpha_{k}=-k_{\perp}^{2} / e B$. Here, $L_{l}^{a}(z)$ denotes the generalized Laguerre polynomial with $L_{-1}^{a}(z)=0$ and $L_{l}(z)=L_{l}^{0}(z)$. We now rewrite Eqs. (24) and (25) using Eqs. (30) and (31) as

$$
\begin{aligned}
D_{B}^{11}(k)= & \sum_{l=0}^{\infty} 2(-1)^{l} e^{-\alpha_{k}} L_{l}\left(2 \alpha_{k}\right) \\
& \times\left[\frac{-1}{k_{\|}^{2}-m_{l}^{2}+i \epsilon}+2 \pi i \eta(k \cdot u) \delta\left(k_{\|}^{2}-m_{l}^{2}\right)\right] \\
S_{B}^{11}(k)= & \sum_{l=0}^{\infty}(-1)^{l} e^{-\alpha_{k}} \mathcal{D}_{l}(k) \\
& \times\left[\frac{-1}{k_{\|}^{2}-M_{l}^{2}+i \epsilon}-2 \pi i \tilde{\eta}(k \cdot u) \delta\left(k_{\|}^{2}-M_{l}^{2}\right)\right],
\end{aligned}
$$

where we have defined the Landau level-dependent "dimensionally reduced effective masses" (as a consequence of dimensional reduction) of the pion and proton as

$m_{l}=\sqrt{m_{\pi}^{2}+(2 l+1) e B}$ and $M_{l}=\sqrt{m_{N}^{2}+2 l e B}$.

We now replace the 11-component of the charged pion and proton propagators in Eqs. (14) and (15) as $D^{11} \rightarrow D_{B}^{11}, S_{\mathrm{p}}^{11} \rightarrow S_{B}^{11}$, i.e., by the respective magnetized ones given in Eqs. (33) and (34), and then perform the $d k^{0}$ integrations (using the Dirac delta functions). Following Eqs. (16) and (17), we get the thermal self-energy functions under external magnetic field which we will denote by a double bar to distinguish them from the thermal self-energy functions in the absence of magnetic field. Their explicit expressions are given by

$$
\begin{aligned}
\operatorname{Re} \overline{\bar{\Pi}}_{\pi}^{\mu \nu}\left(q^{0}, \vec{q}\right) & =\sum_{l=0}^{\infty} \sum_{n=0}^{\infty} \int \frac{d^{3} k}{(2 \pi)^{3}} \mathcal{P}\left[\frac{f\left(\omega_{k}^{l}\right)}{2 \omega_{k}^{l}}\left\{\frac{\mathcal{N}_{\pi, n l}^{\mu \nu}\left(k^{0}=-\omega_{k}^{l}\right)}{\left(q^{0}-\omega_{k}^{l}\right)^{2}-\left(\omega_{p}^{n}\right)^{2}}+\frac{\mathcal{N}_{\pi, n l}^{\mu \nu}\left(k^{0}=\omega_{k}^{l}\right)}{\left(q^{0}+\omega_{k}^{l}\right)^{2}-\left(\omega_{p}^{n}\right)^{2}}\right\}\right. \\
& \left.+\frac{f\left(\omega_{p}^{n}\right)}{2 \omega_{p}^{n}}\left\{\frac{\mathcal{N}_{\pi, n l}^{\mu \nu}\left(k^{0}=-q^{0}-\omega_{p}^{n}\right)}{\left(q^{0}+\omega_{p}^{n}\right)^{2}-\left(\omega_{k}^{l}\right)^{2}}+\frac{\mathcal{N}_{\pi, n l}^{\mu \nu}\left(k^{0}=-q^{0}+\omega_{p}^{n}\right)}{\left(q^{0}-\omega_{p}^{n}\right)^{2}-\left(\omega_{k}^{l}\right)^{2}}\right\}\right]+\operatorname{Re}\left(\Pi_{\pi}^{\mu \nu}\right)_{\mathrm{vac}}(q, e B) \\
\operatorname{Re} \overline{\bar{\Pi}}_{\mathrm{N}}^{\mu \nu}\left(q^{0}, \vec{q}\right) & =\frac{1}{2} \operatorname{Re} \bar{\Pi}_{\mathrm{N}}^{\mu \nu}\left(q^{0}, \vec{q}\right)-\sum_{l=0}^{\infty} \sum_{n=0}^{\infty} \int \frac{d^{3} k}{(2 \pi)^{3}} \mathcal{P}\left[\frac{1}{2 \Omega_{k}^{l}}\left\{\frac{f^{-}\left(\Omega_{k}^{l}\right) \mathcal{N}_{\mathrm{p}, n l}^{\mu \nu}\left(k^{0}=-\Omega_{k}^{l}\right)}{\left(q^{0}-\Omega_{k}^{l}\right)^{2}-\left(\Omega_{p}^{n}\right)^{2}}+\frac{f^{+}\left(\Omega_{k}^{l}\right) \mathcal{N}_{\mathrm{p}, n l}^{\mu \nu}\left(k^{0}=\Omega_{k}^{l}\right)}{\left(q^{0}+\Omega_{k}^{l}\right)^{2}-\left(\Omega_{p}^{n}\right)^{2}}\right\}\right. \\
& \left.+\frac{1}{2 \Omega_{p}^{n}}\left\{\frac{f^{-}\left(\Omega_{p}^{n}\right) \mathcal{N}_{\mathrm{p}, n l}^{\mu \nu}\left(k^{0}=-q^{0}-\Omega_{p}^{n}\right)}{\left(q^{0}+\Omega_{p}^{n}\right)^{2}-\left(\Omega_{k}^{l}\right)^{2}}+\frac{f^{+}\left(\Omega_{p}^{n}\right) \mathcal{N}_{\mathrm{p}, n l}^{\mu \nu}\left(k^{0}=-q^{0}+\Omega_{p}^{n}\right)}{\left(q^{0}-\Omega_{p}^{n}\right)^{2}-\left(\Omega_{k}^{l}\right)^{2}}\right\}\right]+\operatorname{Re}\left(\Pi_{\mathrm{p}}^{\mu \nu}\right)_{\mathrm{vac}}(q, e B)
\end{aligned}
$$




$$
\begin{aligned}
\operatorname{Im} \overline{\bar{\Pi}}_{\pi}^{\mu \nu}\left(q^{0}, \vec{q}\right)= & -\operatorname{sign}\left(q^{0}\right) \tanh \left(\frac{\beta q^{0}}{2}\right) \pi \sum_{l=0}^{\infty} \sum_{n=0}^{\infty} \int \frac{d^{3} k}{(2 \pi)^{3}} \frac{1}{4 \omega_{k}^{l} \omega_{p}^{n}}\left[\left\{1+f\left(\omega_{k}^{l}\right)+f\left(\omega_{p}^{n}\right)+2 f\left(\omega_{k}^{l}\right) f\left(\omega_{p}^{n}\right)\right\}\right. \\
& \left\{\mathcal{N}_{\pi, n l}^{\mu \nu}\left(k^{0}=-\omega_{k}^{l}\right) \delta\left(q^{0}-\omega_{k}^{l}-\omega_{p}^{n}\right)+\mathcal{N}_{\pi, n l}^{\mu \nu}\left(k^{0}=\omega_{k}^{l}\right) \delta\left(q^{0}+\omega_{k}^{l}+\omega_{p}^{n}\right)\right\}+\left\{f\left(\omega_{k}^{l}\right)+f\left(\omega_{p}^{n}\right)+2 f\left(\omega_{k}^{l}\right) f\left(\omega_{p}^{n}\right)\right\} \\
& \left.\left\{\mathcal{N}_{\pi, n l}^{\mu \nu}\left(k^{0}=-\omega_{k}^{l}\right) \delta\left(q^{0}-\omega_{k}^{l}+\omega_{p}^{n}\right)+\mathcal{N}_{\pi, n l}^{\mu \nu}\left(k^{0}=\omega_{k}^{l}\right) \delta\left(q^{0}+\omega_{k}^{l}-\omega_{p}^{n}\right)\right\}\right]
\end{aligned}
$$

$$
\begin{aligned}
\operatorname{Im} \overline{\bar{\Pi}}_{\mathrm{N}}^{\mu \nu}\left(q^{0}, \vec{q}\right)= & \frac{1}{2} \operatorname{Im} \bar{\Pi}_{\mathrm{N}}^{\mu \nu}\left(q^{0}, \vec{q}\right)-\operatorname{sign}\left(q^{0}\right) \tanh \left(\frac{\beta q^{0}}{2}\right) \pi \sum_{l=0}^{\infty} \sum_{n=0}^{\infty} \int \frac{d^{3} k}{(2 \pi)^{3}} \frac{1}{4 \Omega_{k}^{l} \Omega_{p}^{n}} \\
& \times\left[\left\{1-f^{-}\left(\Omega_{k}^{l}\right)-f^{+}\left(\Omega_{p}^{n}\right)+2 f^{-}\left(\Omega_{k}^{l}\right) f^{+}\left(\Omega_{p}^{n}\right)\right\} \mathcal{N}_{\mathrm{p}, n l}^{\mu \nu}\left(k^{0}=-\Omega_{k}^{l}\right) \delta\left(q^{0}-\Omega_{k}^{l}-\Omega_{p}^{n}\right)\right. \\
& +\left\{1-f^{+}\left(\Omega_{k}^{l}\right)-f^{-}\left(\Omega_{p}^{n}\right)+2 f^{+}\left(\Omega_{k}^{l}\right) f^{-}\left(\Omega_{p}^{n}\right)\right\} \mathcal{N}_{\mathrm{p}, n l}^{\mu \nu}\left(k^{0}=\Omega_{k}^{l}\right) \delta\left(q^{0}+\Omega_{k}^{l}+\Omega_{p}^{n}\right) \\
& +\left\{-f^{-}\left(\Omega_{k}^{l}\right)-f^{-}\left(\Omega_{p}^{n}\right)+2 f^{-}\left(\Omega_{k}^{l}\right) f^{-}\left(\Omega_{p}^{n}\right)\right\} \mathcal{N}_{\mathrm{p}, n l}^{\mu \nu}\left(k^{0}=-\omega_{k}^{l}\right) \delta\left(q^{0}-\Omega_{k}^{l}+\Omega_{p}^{n}\right) \\
& \left.+\left\{-f^{+}\left(\Omega_{k}^{l}\right)-f^{+}\left(\Omega_{p}^{n}\right)+2 f^{+}\left(\Omega_{k}^{l}\right) f^{+}\left(\Omega_{p}^{n}\right)\right\} \mathcal{N}_{\mathrm{p}, n l}^{\mu \nu}\left(k^{0}=\Omega_{k}^{l}\right) \delta\left(q^{0}+\Omega_{k}^{l}-\Omega_{p}^{n}\right)\right],
\end{aligned}
$$

where

$$
\mathcal{N}_{\pi, n l}^{\mu \nu}(q, k)=4(-1)^{n+l} e^{-\alpha_{k}-\alpha_{p}} L_{l}\left(2 \alpha_{k}\right) L_{n}\left(2 \alpha_{p}\right) \mathcal{N}_{\pi}^{\mu \nu}(q, k)
$$

$$
\begin{aligned}
& \mathcal{N}_{\mathrm{p}, n l}^{\mu \nu}(q, k)=-g_{\rho N N}^{2}(-1)^{n+l} e^{-\alpha_{k}-\alpha_{p}} \\
& \times \operatorname{Tr}\left[\Gamma^{\nu}(q) \mathcal{D}_{n}(q+k) \Gamma^{\mu}(-q) \mathcal{D}_{l}(k)\right] \\
& \omega_{k}^{l}= \sqrt{k_{z}^{2}+m_{l}^{2}}=\sqrt{k_{z}^{2}+m_{\pi}^{2}+(2 l+1) e B} \\
& \Omega_{k}^{l}=\sqrt{k_{z}^{2}+M_{l}^{2}}=\sqrt{k_{z}^{2}+m_{N}^{2}+2 l e B} .
\end{aligned}
$$

The first terms on the rhs of Eqs. (37) and (39) are the contributions from the neutron-neutron loop which are not affected by the external magnetic field. The last terms on the rhs of Eqs. (36) and (37) are the contributions from $\pi \pi$ and proton-proton loop which depend on the external magnetic field but are independent of temperature. Their explicit forms are given by

$$
\begin{aligned}
& \operatorname{Re}\left(\Pi_{\pi}^{\mu \nu}\right)_{\mathrm{vac}}(q, e B) \\
& \quad=\operatorname{Re} \sum_{l=0}^{\infty} \sum_{n=0}^{\infty} i \int \frac{d^{4} k}{(2 \pi)^{4}} \mathcal{N}_{\pi, n l}^{\mu \nu} \Delta_{F}\left(k_{\|}, m_{l}\right) \Delta_{F}\left(q_{\|}+k_{\|}, m_{n}\right) \\
& \operatorname{Re}\left(\Pi_{\mathrm{p}}^{\mu \nu}\right)_{\mathrm{vac}}(q, e B) \\
& \quad=\operatorname{Re} \sum_{l=0}^{\infty} \sum_{n=0}^{\infty} i \int \frac{d^{4} k}{(2 \pi)^{4}} \mathcal{N}_{\mathrm{p}, n l}^{\mu \nu} \Delta_{F}\left(k_{\|}, M_{l}\right) \Delta_{F}\left(q_{\|}+k_{\|}, M_{n}\right)
\end{aligned}
$$

It is important to note that the above quantities respectively contain the divergent pure-vacuum contributions $\left(\Pi_{\pi}^{\mu \nu}\right)_{\text {pure-vac }}(q)$ and $\frac{1}{2}\left(\Pi_{\mathrm{N}}^{\mu \nu}\right)_{\text {pure-vac }}(q)$ in a nontrivial way (as the above equations seem to appear nonperturbative in $e B)$. In contrast, for the case of weak magnetic field expansion of the Schwinger propagator, the pure-vacuum contribution to the self-energy trivially decouples from the magnetic field-dependent terms. Since we are working with the full propagator including all the Landau levels, we have to properly regularize the above expressions in order to extract the pure-vacuum contributions from these quantities. We use dimensional regularization in which the ultraviolet divergence appears as the pole of Gamma and Hurwitz zeta function, the details of which are provided in the Appendixes $C$ and D. Here, we take the transverse momentum of $\rho^{0}$ to be zero, i.e., $q_{\perp}=0$, which makes substantial simplifications of the analytic calculations. The final result can be read off from Eqs. (C8) and (D8) as

$$
\left(\Pi_{\pi}^{\mu \nu}\right)_{\mathrm{vac}}\left(q_{\|}, e B\right)=\left(\Pi_{\pi}^{\mu \nu}\right)_{\text {pure-vac }}\left(q_{\|}\right)+\left(\Pi_{\pi}^{\mu \nu}\right)_{e B-\mathrm{vac}}\left(q_{\|}, e B\right)
$$

$$
\left(\Pi_{\mathrm{p}}^{\mu \nu}\right)_{\mathrm{vac}}\left(q_{\|}, e B\right)=\frac{1}{2}\left(\Pi_{\mathrm{N}}^{\mu \nu}\right)_{\text {pure-vac }}\left(q_{\|}\right)+\left(\Pi_{\mathrm{p}}^{\mu \nu}\right)_{e B \text {-vac }}\left(q_{\|}, e B\right)
$$

where the scale-dependent divergent pure-vacuum parts are completely decoupled as the first term on the rhs of the above equation; the scale-independent and finite "eB-dependent vacuum contributions" to the real part of the self-energy functions are 


$$
\begin{aligned}
\left(\Pi_{\pi}^{\mu \nu}\right)_{e B-\mathrm{vac}}\left(q_{\|}, e B\right)= & \frac{-g_{\rho \pi \pi}^{2} q_{\|}^{2}}{32 \pi^{2}} \int_{0}^{1} d x\left[\left\{\ln \left(\frac{\Delta_{\pi}\left(q_{\perp}=0\right)}{2 e B}\right)-1\right\} \Delta_{\pi}\left(q_{\perp}=0\right)\left(q_{\|}^{2} g^{\mu \nu}-q_{\|}^{\mu} q_{\|}^{\nu}\right)\right. \\
& -\left(q_{\|}^{2} g_{\|}^{\mu \nu}-q_{\|}^{\mu} q_{\|}^{\nu}\right) 2 e B\left\{\ln \Gamma\left(z_{\pi}+\frac{1}{2}\right)-\ln \sqrt{2 \pi}\right\} \\
& \left.+q_{\|}^{2} g_{\perp}^{\mu \nu}\left\{\Delta_{\pi}\left(q_{\perp}=0\right)+\frac{e B}{2}-\frac{1}{2} \Delta_{\pi}\left(q_{\perp}=0\right)\left\{\psi\left(z_{\pi}+\frac{1}{2}\right)+\psi\left(z_{\pi}+x+\frac{1}{2}\right)\right\}\right\}\right] \\
\left(\Pi_{\mathrm{p}}^{\mu \nu}\right)_{e B-\mathrm{vac}}\left(q_{\|}, e B\right)= & \frac{g_{\rho N N}^{2}}{4 \pi^{2}} \int_{0}^{1} d x\left[\ln \left(\frac{\Delta_{N}\left(q_{\perp}=0\right)}{2 e B}\right)\left\{2 x(1-x)+\kappa_{\rho}+\frac{\kappa_{\rho}^{2}}{2}-\frac{\kappa_{\rho}^{2}}{4 m_{N}^{2}} \Delta_{N}\left(q_{\perp}=0\right)\right\}\left(q_{\|}^{2} g^{\mu \nu}-q_{\|}^{\mu} q_{\|}^{\nu}\right)\right. \\
& -2 x(1-x)\left(\psi\left(z_{N}\right)+\frac{1}{2 z_{N}}\right)\left(q_{\|}^{2} g_{\|}^{\mu \nu}-q_{\|}^{\mu} q_{\|}^{\nu}\right)+2 e B g_{\perp}^{\mu \nu}\left\{\left(z_{N}-\frac{m_{N}^{2}}{e B}\right) \psi\left(z_{N}+x\right)+z_{N}\right. \\
& +\ln \Gamma(z+x)-\ln \sqrt{2 \pi}\}-\kappa_{\rho}\left\{\left(q_{\|}^{2} g_{\|}^{\mu \nu}-q_{\|}^{\mu} q_{\|}^{\nu}\right)\left(\psi\left(z_{N}\right)+\frac{1}{2 z_{N}}\right)+q_{\|}^{2} g_{\perp}^{\mu \nu} \psi(z+x)\right\} \\
& +\frac{\kappa_{\rho}^{2}}{4 m_{N}^{2}} 2 e B\left[\left(q_{\|}^{2} g_{\|}^{\mu \nu}-q_{\|}^{\mu} q_{\|}^{\nu}\right)\left\{-\frac{m_{N}^{2}}{e B}\left(\psi\left(z_{N}\right)+\frac{1}{2 z_{N}}\right)+\frac{1}{2} \ln \left(z_{N}\right)+\ln \Gamma\left(z_{N}\right)-\ln \sqrt{2 \pi}\right\}\right. \\
& \left.-q_{\|}^{2} g_{\perp}^{\mu \nu}\left\{\left(\frac{m_{N}^{2}}{e B}-z_{N}\right) \psi\left(z_{N}+x\right)+\Delta_{N}\left(q_{\perp}=0\right)\right\}+\frac{\kappa_{\rho}^{2}}{4 m_{N}^{2}}\left(q_{\|}^{2} g^{\mu \nu}-q_{\|}^{\mu} q_{\|}^{\nu}\right) \Delta_{N}\left(q_{\perp}=0\right)\right] .
\end{aligned}
$$

Equations (46) and (47) imply that the vacuum counterterms are sufficient to renormalize the theory and thus the external magnetic field does not create additional divergences. For $q_{\perp}=0$, the $d^{2} k_{\perp}$ integrals in Eqs. (36)-(39) can be analytically performed (see Appendix E), and we finally get

$$
\begin{aligned}
\operatorname{Re} \overline{\bar{\Pi}}_{\pi}^{\mu \nu}\left(q^{0}, q_{z}\right) & =\operatorname{Re}\left(\Pi_{\pi}^{\mu \nu}\right)_{\text {pure-vac }}\left(q_{\|}\right)+\sum_{n=0}^{\infty} \sum_{l=(n-1)}^{(n+1)} \int_{-\infty}^{\infty} \frac{d k_{z}}{2 \pi} \mathcal{P}\left[\frac{f\left(\omega_{k}^{l}\right)}{2 \omega_{k}^{l}}\left\{\frac{\tilde{\mathcal{N}}_{\pi, n l}^{\mu \nu}\left(k^{0}=-\omega_{k}^{l}\right)}{\left(q^{0}-\omega_{k}^{l}\right)^{2}-\left(\omega_{p}^{n}\right)^{2}}+\frac{\tilde{\mathcal{N}}_{\pi, n l}^{\mu \nu}\left(k^{0}=\omega_{k}^{l}\right)}{\left(q^{0}+\omega_{k}^{l}\right)^{2}-\left(\omega_{p}^{n}\right)^{2}}\right\}\right. \\
& \left.+\frac{f\left(\omega_{p}^{n}\right)}{2 \omega_{p}^{n}}\left\{\frac{\tilde{\mathcal{N}}_{\pi, n l}^{\mu \nu}\left(k^{0}=-q^{0}-\omega_{p}^{n}\right)}{\left(q^{0}+\omega_{p}^{n}\right)^{2}-\left(\omega_{k}^{l}\right)^{2}}+\frac{\tilde{\mathcal{N}}_{\pi, n l}^{\mu \nu}\left(k^{0}=-q^{0}+\omega_{p}^{n}\right)}{\left(q^{0}-\omega_{p}^{n}\right)^{2}-\left(\omega_{k}^{l}\right)^{2}}\right\}\right]+\operatorname{Re}\left(\Pi_{\pi}^{\mu \nu}\right)_{e B-\operatorname{vac}}\left(q_{\|}, e B\right)
\end{aligned}
$$

$$
\begin{aligned}
\operatorname{Re} \bar{\Pi}_{\mathrm{N}}^{\mu \nu}\left(q^{0}, q_{z}\right)= & \operatorname{Re} \bar{\Pi}_{\mathrm{N}}^{\mu \nu}\left(q^{0}, q_{z}\right)-\sum_{n=0}^{\infty} \sum_{l=(n-1)}^{(n+1)} \int_{-\infty}^{\infty} \frac{d k_{z}}{2 \pi} \mathcal{P}\left[\frac{1}{2 \Omega_{k}^{l}}\left\{\frac{f^{-}\left(\Omega_{k}^{l}\right) \tilde{\mathcal{N}}_{\mathrm{p}, n l}^{\mu \nu}\left(k^{0}=-\Omega_{k}^{l}\right)}{\left(q^{0}-\Omega_{k}^{l}\right)^{2}-\left(\Omega_{p}^{n}\right)^{2}}+\frac{f^{+}\left(\Omega_{k}^{l}\right) \tilde{\mathcal{N}}_{\mathrm{p}, n l}^{\mu \nu}\left(k^{0}=\Omega_{k}^{l}\right)}{\left(q^{0}+\Omega_{k}^{l}\right)^{2}-\left(\Omega_{p}^{n}\right)^{2}}\right\}\right. \\
& +\frac{1}{2 \Omega_{p}^{n}}\left\{\frac{f^{-}\left(\Omega_{p}^{n}\right) \tilde{\mathcal{N}}_{\mathrm{p}, n l}^{\mu \nu}\left(k^{0}=-q^{0}-\Omega_{p}^{n}\right)}{\left(q^{0}+\Omega_{p}^{n}\right)^{2}-\left(\Omega_{k}^{l}\right)^{2}}+\frac{f^{+}\left(\Omega_{p}^{n}\right) \tilde{\mathcal{N}}_{\mathrm{p}, n l}^{\mu \nu}\left(k^{0}=-q^{0}+\Omega_{p}^{n}\right)}{\left(q^{0}-\Omega_{p}^{n}\right)^{2}-\left(\Omega_{k}^{l}\right)^{2}}\right\}+\operatorname{Re}\left(\Pi_{\mathrm{p}}^{\mu \nu}\right)_{e B-\mathrm{vac}}\left(q_{\|}, e B\right)
\end{aligned}
$$

$\operatorname{Im} \overline{\bar{\Pi}}_{\pi}^{\mu \nu}\left(q^{0}, q_{z}\right)=-\operatorname{sign}\left(q^{0}\right) \tanh \left(\frac{\beta q^{0}}{2}\right) \pi \sum_{n=0}^{\infty} \sum_{l=(n-1)}^{(n+1)} \int_{-\infty}^{\infty} \frac{d k_{z}}{2 \pi} \frac{1}{4 \omega_{k}^{l} \omega_{p}^{n}}\left[\left\{1+f\left(\omega_{k}^{l}\right)+f\left(\omega_{p}^{n}\right)+2 f\left(\omega_{k}^{l}\right) f\left(\omega_{p}^{n}\right)\right\}\right.$

$$
\begin{aligned}
& \left\{\tilde{\mathcal{N}}_{\pi, n l}^{\mu \nu}\left(k^{0}=-\omega_{k}^{l}\right) \delta\left(q^{0}-\omega_{k}^{l}-\omega_{p}^{n}\right)+\tilde{\mathcal{N}}_{\pi, n l}^{\mu \nu}\left(k^{0}=\omega_{k}^{l}\right) \delta\left(q^{0}+\omega_{k}^{l}+\omega_{p}^{n}\right)\right\}+\left\{f\left(\omega_{k}^{l}\right)+f\left(\omega_{p}^{n}\right)+2 f\left(\omega_{k}^{l}\right) f\left(\omega_{p}^{n}\right)\right\} \\
& \left.\left\{\tilde{\mathcal{N}}_{\pi, n l}^{\mu \nu}\left(k^{0}=-\omega_{k}^{l}\right) \delta\left(q^{0}-\omega_{k}^{l}+\omega_{p}^{n}\right)+\tilde{\mathcal{N}}_{\pi, n l}^{\mu \nu}\left(k^{0}=\omega_{k}^{l}\right) \delta\left(q^{0}+\omega_{k}^{l}-\omega_{p}^{n}\right)\right\}\right]
\end{aligned}
$$

$\operatorname{Im} \overline{\bar{\Pi}}_{\mathrm{N}}^{\mu \nu}\left(q^{0}, q_{z}\right)=\frac{1}{2} \operatorname{Im} \bar{\Pi}_{\mathrm{N}}^{\mu \nu}\left(q^{0}, q_{z}\right)-\operatorname{sign}\left(q^{0}\right) \tanh \left(\frac{\beta q^{0}}{2}\right) \pi \sum_{n=0}^{\infty} \sum_{l=(n-1)}^{(n+1)} \int_{-\infty}^{\infty} \frac{d k_{z}}{2 \pi} \frac{1}{4 \Omega_{k}^{l} \Omega_{p}^{n}}$

$$
\begin{aligned}
& \times\left[\left\{1-f^{-}\left(\Omega_{k}^{l}\right)-f^{+}\left(\Omega_{p}^{n}\right)+2 f^{-}\left(\Omega_{k}^{l}\right) f^{+}\left(\Omega_{p}^{n}\right)\right\} \tilde{\mathcal{N}}_{\mathrm{p}, n l}^{\mu \nu}\left(k^{0}=-\Omega_{k}^{l}\right) \delta\left(q^{0}-\Omega_{k}^{l}-\Omega_{p}^{n}\right)\right. \\
& +\left\{1-f^{+}\left(\Omega_{k}^{l}\right)-f^{-}\left(\Omega_{p}^{n}\right)+2 f^{+}\left(\Omega_{k}^{l}\right) f^{-}\left(\Omega_{p}^{n}\right)\right\} \tilde{\mathcal{N}}_{\mathrm{p}, n l}^{\mu \nu}\left(k^{0}=\Omega_{k}^{l}\right) \delta\left(q^{0}+\Omega_{k}^{l}+\Omega_{p}^{n}\right) \\
& +\left\{-f^{-}\left(\Omega_{k}^{l}\right)-f^{-}\left(\Omega_{p}^{n}\right)+2 f^{-}\left(\Omega_{k}^{l}\right) f^{-}\left(\Omega_{p}^{n}\right)\right\} \tilde{\mathcal{N}}_{\mathrm{p}, n l}^{\mu \nu}\left(k^{0}=-\omega_{k}^{l}\right) \delta\left(q^{0}-\Omega_{k}^{l}+\Omega_{p}^{n}\right) \\
& \left.+\left\{-f^{+}\left(\Omega_{k}^{l}\right)-f^{+}\left(\Omega_{p}^{n}\right)+2 f^{+}\left(\Omega_{k}^{l}\right) f^{+}\left(\Omega_{p}^{n}\right)\right\} \tilde{\mathcal{N}}_{\mathrm{p}, n l}^{\mu \nu}\left(k^{0}=\Omega_{k}^{l}\right) \delta\left(q^{0}+\Omega_{k}^{l}-\Omega_{p}^{n}\right)\right]
\end{aligned}
$$


where $\tilde{\mathcal{N}}_{\pi, n l}^{\mu \nu}\left(q_{\|}, k_{\|}\right)$and $\tilde{\mathcal{N}}_{\mathrm{p}, n l}^{\mu \nu}\left(q_{\|}, k_{\|}\right)$can be read off from Eqs. (E5) and (E8). The presence of Kronecker delta functions in the expressions of $\tilde{\mathcal{N}}_{\pi, n l}^{\mu \nu}\left(q_{\|}, k_{\|}\right)$and $\tilde{\mathcal{N}}_{\mathrm{p}, n l}^{\mu \nu}\left(q_{\|}, k_{\|}\right)$has eliminated one of the double sums, or in other words, the sum over index $l$ now runs from $(n-1)$ to $(n+1)$.

\section{LORENTZ STRUCTURE OF THE VECTOR BOSON SELF-ENERGY IN MAGNETIZED MEDIUM}

In this section, we will derive the tensorial decomposition of the massive vector boson self-energy. We note that the self-energy $\Pi^{\mu \nu}(q)$, being a second rank tensor, has 16 components which will mix among themselves with the change of frame. It is useful to use linearly independent basis tensors (constructed with the available vectors and tensors) to express $\Pi^{\mu \nu}(q)$ so that the form factors (corresponding to each basis) remain Lorentz invariant. This will also enable one to solve the Dyson-Schwinger equation in order to obtain the complete interacting vector boson propagator. In order to proceed, we first note that the vector boson self-energy satisfies the following constraint:

$$
\Pi^{\mu \nu}(q)=\Pi^{\nu \mu}(q) \quad \text { and } \quad q_{\mu} \Pi^{\mu \nu}(q)=0 .
$$

Let us first consider the pure-vacuum case, i.e., for zero temperature and zero external magnetic field. In this case, the only available vector is the momentum $q^{\mu}$ along with the metric tensor $g^{\mu \nu}$ so that $\Pi^{\mu \nu}(q)$ is a linear combination of $q^{\mu} q^{\nu}$ and $g^{\mu \nu}$, i.e., $\Pi^{\mu \nu}(q)=\left(\alpha_{1} g^{\mu \nu}+\alpha_{2} q^{\mu} q^{\nu}\right)$. Imposing the constrains of Eq. (54), we get $\alpha_{1}+\alpha_{2} q^{2}=0$, which makes the only possible Lorentz structure of the self-energy as

$$
\Pi^{\mu \nu}=\alpha_{1}\left(g^{\mu \nu}-\frac{q^{\mu} q^{\nu}}{q^{2}}\right)
$$

where the Lorentz invariant form factor $\alpha_{1}=\alpha_{1}\left(q^{2}\right)=\frac{1}{3} \Pi^{\mu}{ }_{\mu}$. Note that, with $q^{\mu}$ and $g^{\mu \nu}$, the only possible Lorentz scalar that can be formed by contracting with $\Pi^{\mu \nu}(q)$ is the quantity $g_{\mu \nu} \Pi^{\mu \nu}=\Pi_{\mu}^{\mu}$, implying the existence of only one form factor.

We now consider the case with finite temperature but zero magnetic field. In this case, we have an additional four-vector $u^{\mu}$ (medium four-velocity) along with $q^{\mu}$ and $g^{\mu \nu}$. This makes $\Pi^{\mu \nu}$ be a linear combination of $g^{\mu \nu}, q^{\mu} q^{\nu}$, $u^{\mu} u^{\nu}, q^{\mu} u^{\nu}$, and $q^{\nu} u^{\mu}$, i.e.,

$\Pi^{\mu \nu}(q)=\left(\alpha_{1} g^{\mu \nu}+\alpha_{2} q^{\mu} q^{\nu}+\alpha_{3} u^{\mu} u^{\nu}+\alpha_{4} q^{\mu} u^{\nu}+\alpha_{5} q^{\nu} q^{\mu}\right)$.

However, imposing the constrains in Eq. (54), we find the relationship among the coefficients

$$
\begin{gathered}
\alpha_{5}=\alpha_{4} \\
\alpha_{1}+\alpha_{2} q^{2}+\alpha_{4}(q \cdot u)=0 \\
\alpha_{3}(q \cdot u)+\alpha_{4} q^{2}=0,
\end{gathered}
$$

which makes only two of the coefficients independent. Choosing $\alpha_{1}$ and $\alpha_{2}$ as independent, we get

$$
\begin{aligned}
\Pi^{\mu \nu}(q)= & \alpha_{1}\left[g^{\mu \nu}+\frac{q^{2}}{(q \cdot u)} u^{\mu} u^{\nu}-\frac{1}{(q \cdot u)}\left(q^{\mu} u^{\nu}+q^{\nu} u^{\mu}\right)\right] \\
& +\alpha_{2}\left[q^{\mu} q^{\nu}+\frac{q^{4}}{(q \cdot u)^{2}} u^{\mu} u^{\nu}-\frac{q^{2}}{(q \cdot u)}\left(q^{\mu} u^{\nu}+q^{\nu} u^{\mu}\right)\right],
\end{aligned}
$$

where the Lorentz invariant form factors $\alpha_{1}=\alpha_{1}\left(q^{2}, q \cdot u\right)$ and $\alpha_{2}=\alpha_{2}\left(q^{2}, q \cdot u\right)$ can be obtained by contracting both sides of the above equations with $g_{\mu \nu}$ and $u_{\mu} u_{\nu}$ so that the form factors will become functions of the Lorentz scalars $g_{\mu \nu} \Pi^{\mu \nu}=\Pi^{\mu}{ }_{\mu}$ and $u_{\mu} u_{\nu} \Pi^{\mu \nu}$. Note that, with $q^{\mu}, u^{\mu}$, and $g^{\mu \nu}$, only two possible Lorentz scalars that can be formed by contracting with $\Pi^{\mu \nu}(q)$ are the quantities $\Pi^{\mu}{ }_{\mu}$ and $u_{\mu} u_{\nu} \Pi^{\mu \nu}$, implying the existence of only two form factors. Unlike the pure-vacuum case given in Eq. (55), here, the decomposition of $\Pi^{\mu \nu}$ in Eq. (60) is not unique. As already mentioned, it is useful to construct linearly independent (and mutually orthogonal) basis tensors [note that the basis tensors within square brackets in Eq. (60) are not mutually orthogonal]. One such choice of orthogonal tensor basis could be

$P_{1}^{\mu \nu}=\left(g^{\mu \nu}-\frac{q^{\mu} q^{\nu}}{q^{2}}-\frac{\tilde{u}^{\mu} \tilde{u}^{\nu}}{\tilde{u}^{2}}\right)$ and $P_{2}^{\mu \nu}=\left(\frac{\tilde{u}^{\mu} \tilde{u}^{\nu}}{\tilde{u}^{2}}\right)$

where

$$
\tilde{u}^{\mu}=u^{\mu}-\frac{(q \cdot u)}{q^{2}} q^{\mu}
$$

which is constructed from $u^{\mu}$ by subtracting out its projection along $q^{\mu}$. It is easy to check that $P_{1}^{\mu \nu}$ and $P_{2}^{\mu \nu}$ satisfy all the properties of projection tensors, i.e.,

$$
g_{\alpha \beta} P_{i}^{\mu \alpha} P_{j}^{\beta \nu}=\delta_{i j} P_{i}^{\mu \nu} \quad \text { and } \quad g_{\alpha \beta} g_{\mu \nu} P_{i}^{\mu \alpha} P_{j}^{\beta \nu}=\delta_{i j} .
$$

Therefore, $\Pi^{\mu \nu}$ can be written as

$$
\Pi^{\mu \nu}(q)=\Pi_{1}\left(q^{2}, q \cdot u\right) P_{1}^{\mu \nu}+\Pi_{2}\left(q^{2}, q \cdot u\right) P_{2}^{\mu \nu},
$$

where the form factors are 


$$
\begin{aligned}
& \Pi_{1}\left(q^{2}, q \cdot u\right)=\frac{1}{2}\left(\Pi_{\mu}^{\mu}-\frac{1}{\tilde{u}^{2}} u_{\mu} u_{\nu} \Pi^{\mu \nu}\right) \quad \text { and } \\
& \Pi_{2}\left(q^{2}, q \cdot u\right)=\left(\frac{1}{\tilde{u}^{2}} u_{\mu} u_{\nu} \Pi^{\mu \nu}\right) .
\end{aligned}
$$

Care should be taken when considering the special case like $\vec{q}=\overrightarrow{0}[35]$. To see this, let us consider $q^{i}=|\vec{q}| n^{i}$ so that the spatial components of the projectors at $\vec{q}=\overrightarrow{0}$ become (in the LRF)

$$
P_{1}^{i j}=g^{i j}+n^{i} n^{j} \quad \text { and } \quad P_{2}^{i j}=-n^{i} n^{j} .
$$

This implies that the spatial components of self-energy at vanishing three-momentum

$$
\Pi^{i j}\left(q^{0}, \vec{q}=\overrightarrow{0}\right)=\Pi_{1} g^{i j}+n^{i} n^{j}\left(\Pi_{1}-\Pi_{2}\right)
$$

depend on the direction of $\vec{q}$ even at $|\vec{q}|=0$. This ambiguity is eliminated by setting an additional constraint on the form factors as $\Pi_{1}\left(q^{0}, \vec{q}=\overrightarrow{0}\right)=\Pi_{2}\left(q^{0}, \vec{q}=\overrightarrow{0}\right)$.

Following the same strategy, we now construct a suitable orthogonal tensor basis for the vector boson self-energy at finite temperature under external magnetic field. In this case, we have an additional four-vector $b^{\mu}$ (corresponding to the magnetic field direction) along with $q^{\mu}, u^{\mu}$, and $g^{\mu \nu}$. This makes the symmetric $\Pi^{\mu \nu}$ be a linear combination of seven tensors as

$$
\begin{aligned}
\Pi^{\mu \nu}(q)= & \alpha_{1} g^{\mu \nu}+\alpha_{2} q^{\mu} q^{\nu}+\alpha_{3} u^{\mu} u^{\nu}+\alpha_{4} b^{\mu} b^{\nu} \\
& +\alpha_{5}\left(q^{\mu} u^{\nu}+q^{\nu} u^{\mu}\right)+\alpha_{6}\left(q^{\mu} b^{\nu}+q^{\nu} b^{\mu}\right) \\
& +\alpha_{7}\left(u^{\mu} b^{\nu}+u^{\nu} b^{\mu}\right) .
\end{aligned}
$$

However, imposing the constrains in Eq. (54), we find the following relationship among the coefficients,

$$
\begin{array}{r}
\alpha_{1}+\alpha_{2} q^{2}+\alpha_{5}(q \cdot u)+\alpha_{6}(q \cdot b)=0 \\
\alpha_{3}+\alpha_{5} q^{2}+\alpha_{7}(q \cdot b)=0 \\
\alpha_{4}(q \cdot b)+\alpha_{6} q^{2}+\alpha_{7}(q \cdot u)=0,
\end{array}
$$

which makes only $(7-3=4)$ four of the coefficients independent. The Lorentz invariant form factors $\alpha_{i}=$ $\alpha_{i}\left(q^{2}, q \cdot u, q \cdot b\right)$ with $i=1,2, \ldots, 7$ can be obtained by contracting both sides of the above equations separately with $g_{\mu \nu}, u_{\mu} u_{\nu}, b_{\mu} b_{\nu}$, and $u_{\mu} b_{\nu}$ so that the form factors will become functions of the Lorentz scalars $\Pi_{\mu}^{\mu}, u_{\mu} u_{\nu} \Pi^{\mu \nu}$, $b_{\mu} b_{\nu} \Pi^{\mu \nu}$, and $u_{\mu} b_{\nu} \Pi^{\mu \nu}$. Note that, with $q^{\mu}, u^{\mu}, b^{\mu}$, and $g^{\mu \nu}$, only four possible Lorentz scalars that can be formed by contracting with $\Pi^{\mu \nu}(q)$ are the quantities $\Pi^{\mu}{ }_{\mu}, u_{\mu} u_{\nu} \Pi^{\mu \nu}$, $b_{\mu} b_{\nu} \Pi^{\mu \nu}$, and $u_{\mu} b_{\nu} \Pi^{\mu \nu}$, implying the existence of only four form factors. Like the finite temperature case, here, the decomposition of $\Pi^{\mu \nu}$ is also not unique. One convenient choice of tensor basis could be

$$
\begin{aligned}
& P_{1}^{\mu \nu}=\left(g^{\mu \nu}-\frac{q^{\mu} q^{\nu}}{q^{2}}-\frac{\tilde{u}^{\mu} \tilde{u}^{\nu}}{\tilde{u}^{2}}-\frac{\tilde{b}^{\mu} \tilde{b}^{\nu}}{\tilde{b}^{2}}\right) \\
& P_{2}^{\mu \nu}=\left(\frac{\tilde{u}^{\mu} \tilde{u}^{\nu}}{\tilde{u}^{2}}\right) \\
& P_{3}^{\mu \nu}=\left(\frac{\tilde{b}^{\mu} \tilde{b}^{\nu}}{\tilde{b}^{2}}\right) \\
& Q^{\mu \nu}=\frac{1}{\sqrt{\tilde{u}^{2} \tilde{b}^{2}}}\left(\tilde{u}^{\mu} \tilde{b}^{\nu}+\tilde{u}^{\nu} \tilde{b}^{\mu}\right),
\end{aligned}
$$

where $\tilde{u}^{\mu}$ is defined in Eq. (62) and $\tilde{b}^{\mu}$ is defined as

$$
\tilde{b}^{\mu}=b^{\mu}-\frac{(q \cdot b)}{q^{2}} q^{\mu}-\frac{b \cdot \tilde{u}}{\tilde{u}^{2}} \tilde{u}^{\mu}
$$

The basis tensors in Eqs. (72)-(75) satisfy the following relations:

$$
\begin{aligned}
g_{\alpha \beta} g_{\mu \nu} P_{i}^{\mu \alpha} P_{j}^{\beta \nu} & =\delta_{i j} \\
g_{\alpha \beta} g_{\mu \nu} P_{i}^{\mu \alpha} Q^{\beta \nu} & =0 \\
g_{\alpha \beta} g_{\mu \nu} Q^{\mu \alpha} Q^{\beta \nu} & =2 \\
g_{\alpha \beta} P_{i}^{\mu \alpha} P_{j}^{\beta \nu} & =\delta_{i j} P_{i}^{\mu \nu} \\
g_{\alpha \beta} Q^{\mu \alpha} Q^{\beta \nu} & =P_{2}^{\mu \nu}+P_{3}^{\mu \nu} \\
g_{\alpha \beta} P_{1}^{\mu \alpha} Q^{\beta \nu} & =g_{\alpha \beta} Q^{\mu \alpha} P_{1}^{\beta \nu}=0 \\
g_{\alpha \beta} P_{2}^{\mu \alpha} Q^{\beta \nu} & =g_{\alpha \beta} Q^{\mu \alpha} P_{3}^{\beta \nu}=\frac{\tilde{u}^{\mu} \tilde{b}^{\nu}}{\sqrt{\tilde{u}^{2} \tilde{b}^{2}}} \\
g_{\alpha \beta} P_{3}^{\mu \alpha} Q^{\beta \nu} & =g_{\alpha \beta} Q^{\mu \alpha} P_{2}^{\beta \nu}=\frac{\tilde{u}^{\nu} \tilde{b}^{\mu}}{\sqrt{\tilde{u}^{2} \tilde{b}^{2}}} .
\end{aligned}
$$

Using the basis given in Eqs. (72)-(75), the self-energy at finite temperature under external magnetic field can be written as

$$
\Pi^{\mu \nu}(q)=\Pi_{\alpha} P_{1}^{\mu \nu}+\Pi_{\beta} P_{2}^{\mu \nu}+\Pi_{\gamma} P_{3}^{\mu \nu}+\Pi_{\delta} Q^{\mu \nu},
$$

where the form factors are obtained as

$$
\begin{aligned}
& \Pi_{\beta}=\frac{1}{\tilde{u}^{2}} u_{\mu} u_{\nu} \Pi^{\mu \nu} \\
& \Pi_{\gamma}=\frac{1}{\tilde{b}^{2}}\left[b_{\mu} b_{\nu} \Pi^{\mu \nu}+\frac{(b \cdot \tilde{u})^{2}}{\tilde{u}^{4}} u_{\mu} u_{\nu} \Pi^{\mu \nu}-2 \frac{(b \cdot \tilde{u})}{\tilde{u}^{2}} u_{\mu} b_{\nu} \Pi^{\mu \nu}\right]
\end{aligned}
$$




$$
\begin{aligned}
& \Pi_{\delta}=\frac{1}{\sqrt{\tilde{u}^{2} \tilde{b}^{2}}}\left[u_{\mu} b_{\nu} \Pi^{\mu \nu}-\frac{(b \cdot \tilde{u})}{\tilde{u}^{2}} u_{\mu} u_{\nu} \Pi^{\mu \nu}\right] \\
& \Pi_{\alpha}=\left(\Pi^{\mu}{ }_{\mu}-\Pi_{\beta}-\Pi_{\gamma}\right) .
\end{aligned}
$$

Analogous to the case of only finite temperature, care should be taken while considering the special case $q_{\perp}=0$. To see this, let us consider $q_{\perp}^{i}=\left|\vec{q}_{\perp}\right| n^{i}$ with $i=1,2$ so that the following components of self-energy at vanishing $q_{\perp}$ become (in the LRF)

$$
\begin{aligned}
& \Pi_{i j}\left(q^{0}, q_{\perp}=0, q_{z}\right)=\Pi_{\alpha} g_{i j}+n_{i} n_{j}\left(\Pi_{\alpha}-\Pi_{\gamma}\right) \\
& \Pi_{i 3}\left(q^{0}, q_{\perp}=0, q_{z}\right)=\frac{q^{0}}{\sqrt{q_{\|}^{2}}} n_{i} \Pi_{\delta},
\end{aligned}
$$

which depend on the direction of $\vec{q}_{\perp}$ even at $q_{\perp}=0$. This ambiguity is eliminated by setting additional constraints on the form factors as

$\Pi_{\alpha}\left(q^{0}, q_{\perp}=0, q_{z}\right)=\Pi_{\gamma}\left(q^{0}, q_{\perp}=0, q_{z}\right) \quad$ and

$\Pi_{\delta}\left(q^{0}, q_{\perp}=0, q_{z}\right)=0$.

\section{INTERACTING $\rho$ MESON PROPAGATOR AND ITS LORENTZ STRUCTURE}

Let us first consider the zero temperature and zero magnetic field case for which the complete interacting $\rho$ propagator $D^{\mu \nu}$ is obtained by solving the Dyson-Schwinger equation

$$
D^{\mu \nu}=\Delta^{\mu \nu}-\Delta^{\mu \alpha} \Pi_{\alpha \beta} D^{\beta \nu}
$$

where

$$
\Delta^{\mu \nu}=\left(-g^{\mu \nu}+\frac{q^{\mu} q^{\nu}}{m_{\rho}^{2}}\right) \Delta_{F}\left(q, m_{\rho}\right)
$$

is the free vacuum Feynman propagator and $\Pi^{\mu \nu}$ is the oneloop self-energy of the $\rho$ meson which has the Lorentz structure given in Eq. (55) as

$$
\Pi^{\mu \nu}=\left(g^{\mu \nu}-\frac{q^{\mu} q^{\nu}}{q^{2}}\right) \Pi
$$

with the form factor $\Pi=\frac{1}{3} \Pi^{\mu}{ }_{\mu}$. In order to solve Eq. (93), we rewrite it as

$$
\left(D^{\mu \nu}\right)^{-1}=\left(\Delta^{\mu \nu}\right)^{-1}+\Pi^{\mu \nu},
$$

where $\left(\Delta^{\mu \nu}\right)^{-1}=\left(q^{2}-m_{\rho}^{2}\right) g^{\mu \nu}-q^{\mu} q^{\nu}$, which satisfies $\Delta^{\mu \alpha}\left(\Delta_{\alpha \nu}\right)^{-1}=g^{\mu}{ }_{\nu}$. Substituting $\Pi^{\mu \nu}$ from Eq. (95) in the above equation, we get the inverse of the complete propagator, which can be inverted using the relation $D^{\mu \alpha}\left(D_{\alpha \nu}\right)^{-1}=g^{\mu}{ }_{\nu}$ to obtain the complete propagator as

$D^{\mu \nu}(q)=\left(-g^{\mu \nu}+\frac{q^{\mu} q^{\nu}}{q^{2}}\right)\left(\frac{-1}{q^{2}-m_{\rho}^{2}+\Pi}\right)-\frac{q^{\mu} q^{\nu}}{q^{2} m_{\rho}^{2}}$.

We now consider the case of finite temperature and zero magnetic field. As already mentioned in Sec. III, in RTF of finite temperature field theory, all the two point correlation functions become $2 \times 2$ matrices in thermal space. In this case, the Dyson-Schwinger equation also becomes a matrix equation [35],

$$
\mathbf{D}^{\mu \nu}=\boldsymbol{\Delta}^{\mu \nu}-\boldsymbol{\Delta}^{\mu \alpha} \Pi_{\alpha \beta} \mathbf{D}^{\beta \nu}
$$

Each term of the above equation can be diagonalized in terms of the respective analytic functions (denoted by a bar) so that the above equation becomes an algebraic one,

$$
\bar{D}^{\mu \nu}=\bar{\Delta}^{\mu \nu}-\bar{\Delta}^{\mu \alpha} \bar{\Pi}_{\alpha \beta} \bar{D}^{\beta \nu}
$$

where $\bar{\Delta}^{\mu \nu}=\Delta^{\mu \nu}$. The above equation can be rewritten as

$$
\left(\bar{D}^{\mu \nu}\right)^{-1}=\left(\bar{\Delta}^{\mu \nu}\right)^{-1}+\bar{\Pi}^{\mu \nu} .
$$

In this case, the Lorentz structure of the thermal self-energy function is given in Eq. (64) as

$$
\bar{\Pi}^{\mu \nu}(q)=\Pi_{1}\left(q^{2}, q \cdot u\right) P_{1}^{\mu \nu}+\Pi_{2}\left(q^{2}, q \cdot u\right) P_{2}^{\mu \nu}
$$

where the projection tensors and form factors are respectively defined in Eqs. (61) and (65). Substituting the above equation in Eq. (100), we get the inverse of the complete propagator. In order to obtain the complete propagator, we write

$$
\bar{D}^{\mu \nu}=A_{1} P_{1}^{\mu \nu}+A_{2} P_{2}^{\mu \nu}+\xi q^{\mu} q^{\nu}
$$

and use the relation $\bar{D}^{\mu \alpha}\left(\bar{D}_{\alpha \nu}\right)^{-1}=g^{\mu}{ }_{\nu}$ to extract $A_{1}, A_{2}$, and $\xi$. The final form of the complete interacting thermal propagator is obtained as

$$
\bar{D}^{\mu \nu}=\frac{P_{1}^{\mu \nu}}{q^{2}-m_{\rho}^{2}+\Pi_{1}}+\frac{P_{2}^{\mu \nu}}{q^{2}-m_{\rho}^{2}+\Pi_{2}}-\frac{q^{\mu} q^{\nu}}{q^{2} m_{\rho}^{2}} .
$$

Finally, we consider the case with both finite temperature and external magnetic field. In this case, we need to solve the Dyson-Schwinger equation

$$
\left(\overline{\bar{D}}^{\mu \nu}\right)^{-1}=\left(\bar{\Delta}^{\mu \nu}\right)^{-1}+\overline{\bar{\Pi}}^{\mu \nu},
$$

where a double bar is used to denote the thermal self-energy function and complete propagator under external magnetic field as discussed in Sec. IV. In this case, the Lorentz 
structure of the thermal self-energy function is given in Eq. (85) as

$$
\overline{\bar{\Pi}}^{\mu \nu}(q)=\Pi_{\alpha} P_{1}^{\mu \nu}+\Pi_{\beta} P_{2}^{\mu \nu}+\Pi_{\gamma} P_{3}^{\mu \nu}+\Pi_{\delta} Q^{\mu \nu},
$$

where the basis tensors and form factors are given in Eqs. (72)-(75) and (86)-(89). Substituting the above equation in Eq. (104), we get the inverse of the complete propagator. In order to obtain the complete propagator, we write

$$
\overline{\bar{D}}^{\mu \nu}=A_{\alpha} P_{1}^{\mu \nu}+A_{\beta} P_{2}^{\mu \nu}+A_{\gamma} P_{3}^{\mu \nu}+A_{\delta} Q^{\mu \nu}+\xi q^{\mu} q^{\nu}
$$

and use the relation $\overline{\bar{D}}^{\mu \alpha}\left(\overline{\bar{D}}_{\alpha \nu}\right)^{-1}=g^{\mu}{ }_{\nu}$ to extract the coefficients as

$$
\begin{aligned}
A_{\alpha} & =\frac{1}{q^{2}-m_{\rho}^{2}+\Pi_{\alpha}} \\
A_{\beta} & =\frac{q^{2}-m_{\rho}^{2}+\Pi_{\gamma}}{\left(q^{2}-m_{\rho}^{2}+\Pi_{\gamma}\right)\left(q^{2}-m_{\rho}^{2}+\Pi_{\beta}\right)-\Pi_{\delta}^{2}} \\
A_{\gamma} & =\frac{q^{2}-m_{\rho}^{2}+\Pi_{\beta}}{\left(q^{2}-m_{\rho}^{2}+\Pi_{\beta}\right)\left(q^{2}-m_{\rho}^{2}+\Pi_{\gamma}\right)-\Pi_{\delta}^{2}} \\
A_{\delta} & =\frac{-\Pi_{\delta}}{\left(q^{2}-m_{\rho}^{2}+\Pi_{\beta}\right)\left(q^{2}-m_{\rho}^{2}+\Pi_{\gamma}\right)-\Pi_{\delta}^{2}} \\
\xi & =\frac{-1}{q^{2} m_{\rho}^{2}} .
\end{aligned}
$$

\section{ANALYTIC STRUCTURE OF THE SELF ENERGY}

In this work, we have considered the transverse momentum of the $\rho$ meson to be zero, i.e., $q_{\perp}=0$. As shown in Eq. (92), for the special case $q_{\perp}=0$, the additional constraints to be imposed on the form factors are

$$
\begin{aligned}
& \Pi_{\alpha}\left(q^{0}, q_{\perp}=0, q_{z}\right)=\Pi_{\gamma}\left(q^{0}, q_{\perp}=0, q_{z}\right) \quad \text { and } \\
& \Pi_{\delta}\left(q^{0}, q_{\perp}=0, q_{z}\right)=0 .
\end{aligned}
$$

Using the above constraints, we get from Eqs. (86)-(89)

$$
\begin{aligned}
\Pi_{\alpha} & =\Pi_{\gamma}=\frac{1}{2}\left(\overline{\bar{\Pi}}^{\mu}{ }_{\mu}-\frac{1}{\tilde{u}^{2}} u_{\mu} u_{\nu} \overline{\bar{\Pi}}^{\mu \nu}\right) \\
\Pi_{\beta} & =\frac{1}{\tilde{u}^{2}} u_{\mu} u_{\nu} \overline{\bar{\Pi}}^{\mu \nu} \\
\Pi_{\delta} & =0
\end{aligned}
$$

which imply that we need to calculate only the two quantities $\overline{\bar{\Pi}}^{\mu}{ }_{\mu}$ and $u_{\mu} u_{\nu} \overline{\bar{\Pi}}^{\mu \nu}=\overline{\bar{\Pi}}^{00}$. These are obtained from
Eqs. (50)-(53) by contracting them with $g_{\mu \nu}$ and $u_{\mu} u_{\nu}$. This essentially means replacing $\mathcal{N}^{\mu \nu}$ for all the loops with $\mathcal{N}^{\mu}{ }_{\mu}$ or $\mathcal{N}^{00}$, an explicit list for which has been provided in Appendix F.

Let us now discuss the analytic structure of the selfenergy functions. We first consider the zero magnetic field case. Each imaginary part of the self-energy function for $\pi \pi$ and NN loops as given in Eqs. (22) and (23) contains four Dirac delta functions. These delta functions represent energy-momentum conservation, and they are nonvanishing in a certain kinematic domain. They are termed the unitary-I, unitary-II, Landau-II, and Landau-I cuts as they appear in those equations. The kinematic regions for the unitary-I and unitary-II cuts are given by [35] $\sqrt{\vec{q}^{2}+4 m_{L}^{2}}<q^{0}<\infty$ and $-\infty<q^{0}<-\sqrt{\vec{q}^{2}+4 m_{L}^{2}}$, whereas the same for the two Landau cuts are $\left|q^{0}\right|<|\vec{q}|$, where $m_{L}$ is the mass of the loop particle, i.e., $m_{L}=m_{\pi}$ or $m_{N}$. These cuts correspond to different physical processes such as decay or scattering. For example, unitary cuts correspond to the decay of $\rho^{0}$ into a $\pi^{+} \pi^{-}$or $N \bar{N}$ pair, and the Landau cuts correspond to the scattering of a $\rho^{0}$ with a pion or nucleon producing the same in the final state along with their time reversed processes. If we restrict ourselves to the physical timelike kinematic regions defined in terms of $q^{0}>0$ and $q^{2}>0$, then only the unitary-I cut contributes. It is important to note that a nontrivial Landau cut appears in the physical timelike region only if the loop particles have different masses and lie in the kinematic domain $|\vec{q}|<q^{0}<\sqrt{\vec{q}^{2}+\Delta m^{2}}$ where $\Delta m$ is the mass difference of the loop particles.

Let us now consider the case of both finite temperature and nonzero external magnetic field. In this case, the imaginary parts of the self-energy as given in Eqs. (52) and (53) also contain four Dirac delta functions corresponding to the unitary and Landau cuts. It is important to note that the arguments of the delta functions contain only the longitudinal dynamics (because of dimensional reduction), which implies that the analytic structure of the selfenergy functions will only depend on the longitudinal momentum of $\rho$. On the other hand, the transverse dynamics has appeared as Landau level-dependent dimensionally reduced effective mass to the loop particles as given in Eq. (35). Therefore, even if the loop particles have the same masses, a nontrivial Landau cut may appear in the physical timelike kinematic domain if the two loop particles reside in different Landau levels. Physically, this means that $\rho^{0}$ can get absorbed in a scattering with a pion or a proton in a lower Landau level, producing another pion or proton in a higher Landau level as the final state. Detailed discussions on the analytic structure in the presence of external magnetic field can be found in Refs. [31,38]. The unitary-I and unitary-II terms for the $\pi \pi$ loop are nonvanishing in the kinematic domains $\sqrt{q_{z}^{2}+4\left(m_{\pi}^{2}+e B\right)}<$ $q^{0}<\infty$ and $-\infty<q^{0}<-\sqrt{q_{z}^{2}+4\left(m_{\pi}^{2}+e B\right)}$, whereas the kinematic domain for both the Landau cuts is 


$$
\left|q^{0}\right|<\sqrt{q_{z}^{2}+\left(\sqrt{m_{\pi}^{2}+e B}-\sqrt{m_{\pi}^{2}+3 e B}\right)^{2}} .
$$

The corresponding kinematic domains for the NN loop are $\sqrt{q_{z}^{2}+4 m_{N}^{2}}<q^{0}<\infty$ and $-\infty<q^{0}<-\sqrt{q_{z}^{2}+4 m_{N}^{2}}$ for the unitary-I and unitary-II cuts respectively and

$$
\left|q^{0}\right|<\sqrt{q_{z}^{2}+\left(m_{N}-\sqrt{m_{N}^{2}+2 e B}\right)^{2}}
$$

for the Landau cuts. Note that the threshold of the Landau cuts appears when the dimensionally reduced effective mass difference between the loop particles is the maximum. As can be seen from Eqs. (52) and (53), for a particular value of the index $n$, the sum over the index $l$ runs only for three values $(n-1), n$, and $(n+1)$, which implies that the Landau level difference between the loop particles can be at most 1 . Thus, the maximum difference in their dimensionally reduced effective mass appears when one of them is at the lowest Landau level and the other one is at the first Landau level, which in turn defines the Landau cut threshold in Eqs. (116) and (117).

We now simplify the expressions of the imaginary parts given in Eqs. (22), (23), (52), and (53) by evaluating one of the integrals using the Dirac delta functions. For the imaginary parts at zero magnetic field, we evaluate the $d(\cos \theta)$ integrals and get (after imposing the kinematic restrictions discussed above)

$$
\begin{aligned}
\operatorname{Im} \bar{\Pi}_{\pi, \mathrm{N}}^{\mu \nu}\left(q^{0}, \vec{q}\right)= & -\operatorname{sign}\left(q^{0}\right) \tanh \left(\frac{q^{0}}{2 T}\right) \frac{1}{16 \pi|\vec{q}|}\left[\int_{\omega_{-}}^{\omega_{+}} d\left(\omega_{k}, \Omega_{k}\right)\left(U_{1}^{\pi, \mathrm{N}}\right)^{\mu \nu}\left(\cos \theta=\cos \theta_{0}^{\pi, \mathrm{N}}\right) \Theta\left(q^{0}-\sqrt{\vec{q}^{2}+4 m_{\pi, \mathrm{N}}^{2}}\right)\right. \\
& +\int_{-\omega_{+}}^{-\omega_{-}} d \omega_{k}\left(U_{2}^{\pi, \mathrm{N}}\right)^{\mu \nu}\left(\cos \theta=\cos \theta_{0}^{\prime \pi, \mathrm{N}}\right) \Theta\left(-q^{0}-\sqrt{\vec{q}^{2}+4 m_{\pi, \mathrm{N}}^{2}}\right) \\
& +\int_{-\omega_{+}}^{\infty} d \omega_{k}\left(L_{1}^{\pi, \mathrm{N}}\right)^{\mu \nu}\left(\cos \theta=\cos \theta_{0}^{\prime \pi, \mathrm{N}}\right) \Theta\left(-\left|q^{0}\right|+|\vec{q}|\right) \\
& \left.+\int_{\omega_{-}}^{\infty} d \omega_{k}\left(L_{2}^{\pi, \mathrm{N}}\right)^{\mu \nu}\left(\cos \theta=\cos \theta_{0}^{\pi, \mathrm{N}}\right) \Theta\left(-\left|q^{0}\right|+|\vec{q}|\right)\right],
\end{aligned}
$$

where

$$
\omega_{ \pm}= \begin{cases}\frac{1}{2 q^{2}}\left[q^{0} q^{2} \pm|\vec{q}| \lambda^{1 / 2}\left(q^{2}, m_{\pi}^{2}, m_{\pi}^{2}\right)\right] & \text { for } \pi \pi \text { loop } \\ \frac{1}{2 q^{2}}\left[q^{0} q^{2} \pm|\vec{q}| \lambda^{1 / 2}\left(q^{2}, m_{N}^{2}, m_{N}^{2}\right)\right] & \text { for NN loop }\end{cases}
$$

$$
\begin{aligned}
\left(U_{1}^{\pi}\right)^{\mu \nu}= & \left\{1+f\left(\omega_{k}\right)+f\left(\omega_{p}\right)+2 f\left(\omega_{k}\right) f\left(\omega_{p}\right)\right\} \\
& \times N_{\pi}^{\mu \nu}\left(k^{0}=-\omega_{k}\right),
\end{aligned}
$$

$$
\begin{aligned}
\left(U_{2}^{\pi}\right)^{\mu \nu}= & \left\{1+f\left(\omega_{k}\right)+f\left(\omega_{p}\right)+2 f\left(\omega_{k}\right) f\left(\omega_{p}\right)\right\} \\
& \times N_{\pi}^{\mu \nu}\left(k^{0}=\omega_{k}\right),
\end{aligned}
$$

$\left(L_{1}^{\pi}\right)^{\mu \nu}=\left\{f\left(\omega_{k}\right)+f\left(\omega_{p}\right)+2 f\left(\omega_{k}\right) f\left(\omega_{p}\right)\right\} N_{\pi}^{\mu \nu}\left(k^{0}=\omega_{k}\right)$,

$\left(L_{2}^{\pi}\right)^{\mu \nu}=\left\{f\left(\omega_{k}\right)+f\left(\omega_{p}\right)+2 f\left(\omega_{k}\right) f\left(\omega_{p}\right)\right\} N_{\pi}^{\mu \nu}\left(k^{0}=-\omega_{k}\right)$,

$$
\begin{aligned}
\left(U_{1}^{\mathrm{N}}\right)^{\mu \nu}= & \left\{1-f^{-}\left(\Omega_{k}\right)-f^{+}\left(\Omega_{p}\right)+2 f^{-}\left(\Omega_{k}\right) f^{+}\left(\Omega_{p}\right)\right\} \\
& \times N_{\mathrm{N}}^{\mu \nu}\left(k^{0}=-\Omega_{k}\right),
\end{aligned}
$$

$$
\begin{aligned}
\left(U_{2}^{\mathrm{N}}\right)^{\mu \nu}= & \left\{1-f^{+}\left(\Omega_{k}\right)-f^{-}\left(\Omega_{p}\right)+2 f^{+}\left(\Omega_{k}\right) f^{-}\left(\Omega_{p}\right)\right\} \\
& \times N_{\mathrm{N}}^{\mu \nu}\left(k^{0}=\Omega_{k}\right) \\
\left(L_{1}^{\mathrm{N}}\right)^{\mu \nu}= & \left\{-f^{+}\left(\Omega_{k}\right)-f^{+}\left(\Omega_{p}\right)+2 f^{+}\left(\Omega_{k}\right) f^{+}\left(\Omega_{p}\right)\right\} \\
& \times N_{\mathrm{N}}^{\mu \nu}\left(k^{0}=\Omega_{k}\right), \\
\left(L_{2}^{\mathrm{N}}\right)^{\mu \nu}= & \left\{-f^{-}\left(\Omega_{k}\right)-f^{-}\left(\Omega_{p}\right)+2 f^{-}\left(\Omega_{k}\right) f^{-}\left(\Omega_{p}\right)\right\} \\
& \times N_{\mathrm{N}}^{\mu \nu}\left(k^{0}=-\Omega_{k}\right) \\
\cos \theta_{0}^{\pi}= & \left(\frac{-2 q^{0} \omega_{k}+q^{2}}{2|\vec{q}||\vec{k}|}\right) \\
\cos \theta_{0}^{\prime \pi}= & \left(\frac{2 q^{0} \omega_{k}+q^{2}}{2|\vec{q}||\vec{k}|}\right), \\
\cos \theta_{0}^{\mathrm{N}}= & \left(\frac{-2 q^{0} \Omega_{k}+q^{2}}{2|\vec{q}||\vec{k}|}\right)
\end{aligned}
$$

and

$\cos \theta_{0}^{\prime N}=\left(\frac{2 q^{0} \Omega_{k}+q^{2}}{2|\vec{q}||\vec{k}|}\right)$ 
with $\lambda(x, y, z)=x^{2}+y^{2}+z^{2}-2 x y-2 y z-2 z x$ being the Källén function.

For the imaginary parts at finite magnetic field, we evaluate the $d k_{z}$ integrals in Eqs. (52) and (53) using the Dirac delta functions. The imaginary part due to the $\pi \pi$ loop simplifies to

$$
\begin{aligned}
\operatorname{Im} \overline{\bar{\Pi}}_{\pi}^{\mu \nu}\left(q^{0}, q_{z}\right)= & -\operatorname{sign}\left(q^{0}\right) \tanh \left(\frac{q^{0}}{2 T}\right) \sum_{n=0}^{\infty} \sum_{l=(n-1)}^{(n+1)} \frac{1}{4 \lambda^{1 / 2}\left(q_{\|}^{2}, m_{l}^{2}, m_{n}^{2}\right)} \sum_{\tilde{k}_{z} \in \tilde{k}_{z}^{ \pm}}\left[\left(\tilde{U}_{1, n l}^{\pi}\right)^{\mu \nu}\left(k_{z}=\tilde{k}_{z}\right) \Theta\left(q^{0}-\sqrt{q_{z}^{2}+\left(m_{l}+m_{n}\right)^{2}}\right)\right. \\
& +\left(\tilde{U}_{2, n l}^{\pi}\right)^{\mu \nu}\left(k_{z}=\tilde{k}_{z}\right) \Theta\left(-q^{0}-\sqrt{q_{z}^{2}+\left(m_{l}+m_{n}\right)^{2}}\right) \\
& +\left(\tilde{L}_{1, n l}^{\pi}\right)^{\mu \nu}\left(k_{z}=\tilde{k}_{z}\right) \Theta\left(q^{0}-\min \left(q_{z}, E_{ \pm}\right)\right) \Theta\left(-q^{0}+\max \left(q_{z}, E_{ \pm}\right)\right) \\
& \left.+\left(\tilde{L}_{2, n l}^{\pi}\right)^{\mu \nu}\left(k_{z}=\tilde{k}_{z}\right) \Theta\left(-q^{0}-\min \left(q_{z}, E_{ \pm}\right)\right) \Theta\left(q^{0}+\max \left(q_{z}, E_{ \pm}\right)\right)\right],
\end{aligned}
$$

where

$$
\begin{aligned}
\left(\tilde{U}_{1, n l}^{\pi}\right)^{\mu \nu}= & \left\{1+f\left(\tilde{\omega}_{k}^{l}\right)+f\left(\tilde{\omega}_{p}^{n}\right)+2 f\left(\tilde{\omega}_{k}^{l}\right) f\left(\tilde{\omega}_{p}^{n}\right)\right\} \\
& \times \tilde{N}_{\pi, n l}^{\mu \nu}\left(k^{0}=-\tilde{\omega}_{k}^{l}\right), \\
\left(\tilde{U}_{1, n l}^{\pi}\right)^{\mu \nu}= & \left\{1+f\left(\tilde{\omega}_{k}^{l}\right)+f\left(\tilde{\omega}_{p}^{n}\right)+2 f\left(\tilde{\omega}_{k}^{l}\right) f\left(\tilde{\omega}_{p}^{n}\right)\right\} \\
& \times \tilde{N}_{\pi, n l}^{\mu \nu}\left(k^{0}=\tilde{\omega}_{k}^{l}\right), \\
\left(\tilde{U}_{1, n l}^{\pi}\right)^{\mu \nu}= & \left\{f\left(\tilde{\omega}_{k}^{l}\right)+f\left(\tilde{\omega}_{p}^{n}\right)+2 f\left(\tilde{\omega}_{k}^{l}\right) f\left(\tilde{\omega}_{p}^{n}\right)\right\} \\
& \times \tilde{N}_{\pi, n l}^{\mu \nu}\left(k^{0}=\tilde{\omega}_{k}^{l}\right),
\end{aligned}
$$

$$
\begin{aligned}
\left(\tilde{U}_{1, n l}^{\pi}\right)^{\mu \nu}= & \left\{f\left(\tilde{\omega}_{k}^{l}\right)+f\left(\tilde{\omega}_{p}^{n}\right)+2 f\left(\tilde{\omega}_{k}^{l}\right) f\left(\tilde{\omega}_{p}^{n}\right)\right\} \\
& \times \tilde{N}_{\pi, n l}^{\mu \nu}\left(k^{0}=-\tilde{\omega}_{k}^{l}\right),
\end{aligned}
$$

with $\quad \tilde{k}_{z}^{ \pm}=\frac{1}{2 q_{\|}^{2}}\left[-y q_{z} \pm\left|q^{0}\right| \lambda^{1 / 2}\left(q_{\|}^{2}, m_{l}^{2}, m_{n}^{2}\right)\right], \quad y=$ $\left(q_{\|}^{2}+m_{l}^{2}-m_{n}^{2}\right), \quad \tilde{\omega}_{k}^{l}=\sqrt{\tilde{k}_{z}^{2}+m_{l}^{2}}, \quad$ and $\quad E_{ \pm}=$ $\frac{m_{l}-m_{n}}{\left|m_{l} \pm m_{n}\right|} \sqrt{q_{z}^{2}+\left(m_{l} \pm m_{n}\right)^{2}}$.

The corresponding expression of the imaginary part due to the NN loop reads

$$
\begin{aligned}
\operatorname{Im} \overline{\bar{\Pi}}_{\mathrm{N}}^{\mu \nu}\left(q^{0}, q_{z}\right)= & \frac{1}{2} \operatorname{Im} \bar{\Pi}_{\mathrm{N}}^{\mu \nu}\left(q^{0}, q_{z}\right)-\operatorname{sign}\left(q^{0}\right) \tanh \left(\frac{q^{0}}{2 T}\right) \sum_{n=0}^{\infty} \sum_{l=(n-1)}^{(n+1)} \frac{1}{4 \lambda^{1 / 2}\left(q_{\|}^{2}, M_{l}^{2}, M_{n}^{2}\right)} \\
& \times \sum_{\tilde{k}_{z} \in \tilde{K}_{z}^{ \pm}}\left[\left(\tilde{U}_{1, n l}^{\mathrm{p}}\right)^{\mu \nu}\left(k_{z}=\tilde{k}_{z}\right) \Theta\left(q^{0}-\sqrt{q_{z}^{2}+\left(M_{l}+M_{n}\right)^{2}}\right)\right. \\
& +\left(\tilde{U}_{2, n l}^{\mathrm{p}}\right)^{\mu \nu}\left(k_{z}=\tilde{k}_{z}\right) \Theta\left(-q^{0}-\sqrt{q_{z}^{2}+\left(M_{l}+M_{n}\right)^{2}}\right) \\
& +\left(\tilde{L}_{1, n l}^{\mathrm{p}}\right)^{\mu \nu}\left(k_{z}=\tilde{k}_{z}\right) \Theta\left(q^{0}-\min \left(q_{z}, E_{ \pm}^{\prime}\right)\right) \Theta\left(-q^{0}+\max \left(q_{z}, E_{ \pm}^{\prime}\right)\right) \\
& \left.+\left(\tilde{L}_{2, n l}^{\mathrm{p}}\right)^{\mu \nu}\left(k_{z}=\tilde{k}_{z}\right) \Theta\left(-q^{0}-\min \left(q_{z}, E_{ \pm}^{\prime}\right)\right) \Theta\left(q^{0}+\max \left(q_{z}, E_{ \pm}^{\prime}\right)\right)\right]
\end{aligned}
$$

where

$$
\begin{aligned}
\left(\tilde{U}_{1, n l}^{\mathrm{p}}\right)^{\mu \nu}= & \left\{1-f^{-}\left(\tilde{\Omega}_{k}^{l}\right)-f^{+}\left(\tilde{\Omega}_{p}^{n}\right)+2 f^{-}\left(\tilde{\Omega}_{k}^{l}\right) f^{+}\left(\tilde{\Omega}_{p}^{n}\right)\right\} \\
& \times \tilde{N}_{\mathrm{p}, n l}^{\mu \nu}\left(k^{0}=-\tilde{\Omega}_{k}^{l}\right), \\
\left(\tilde{U}_{1, n l}^{\mathrm{p}}\right)^{\mu \nu}= & \left\{1-f^{+}\left(\tilde{\Omega}_{k}^{l}\right)-f^{-}\left(\tilde{\Omega}_{p}^{n}\right)+2 f^{+}\left(\tilde{\Omega}_{k}^{l}\right) f^{-}\left(\tilde{\Omega}_{p}^{n}\right)\right\} \\
& \times \tilde{N}_{\mathrm{p}, n l}^{\mu \nu}\left(k^{0}=\tilde{\Omega}_{k}^{l}\right), \\
\left(\tilde{U}_{1, n l}^{\mathrm{p}}\right)^{\mu \nu}= & \left\{-f^{+}\left(\tilde{\Omega}_{k}^{l}\right)-f^{+}\left(\tilde{\Omega}_{p}^{n}\right)+2 f\left(\tilde{\Omega}_{k}^{l}\right) f\left(\tilde{\Omega}_{p}^{n}\right)\right\} \\
& \times \tilde{N}_{\mathrm{p}, n l}^{\mu \nu}\left(k^{0}=\tilde{\Omega}_{k}^{l}\right),
\end{aligned}
$$

$$
\begin{aligned}
\left(\tilde{U}_{1, n l}^{\mathrm{p}}\right)^{\mu \nu}= & \left\{-f^{-}\left(\tilde{\Omega}_{k}^{l}\right)-f^{-}\left(\tilde{\Omega}_{p}^{n}\right)+2 f\left(\tilde{\Omega}_{k}^{l}\right) f\left(\tilde{\Omega}_{p}^{n}\right)\right\} \\
& \times \tilde{N}_{\mathrm{p}, n l}^{\mu \nu}\left(k^{0}=-\tilde{\Omega}_{k}^{l}\right)
\end{aligned}
$$

with $\quad \tilde{K}_{z}^{ \pm}=\frac{1}{2 q_{\|}^{2}}\left[-Y q_{z} \pm\left|q^{0}\right| \lambda^{1 / 2}\left(q_{\|}^{2}, M_{l}^{2}, M_{n}^{2}\right)\right], \quad Y=$ $\left(q_{\|}^{2}+M_{l}^{2}-M_{n}^{2}\right), \quad \tilde{\Omega}_{k}^{l}=\sqrt{\tilde{K}_{z}^{2}+M_{l}^{2}}, \quad$ and $\quad E_{ \pm}^{\prime}=$ $\frac{M_{l}-M_{n}}{\left|M_{l} \pm M_{n}\right|} \sqrt{q_{z}^{2}+\left(M_{l} \pm M_{n}\right)^{2}}$. The first term on the rhs of Eq. (137) is the contribution from the neutron-neutron loop (which is not affected by the external magnetic field) of which the simplified form is given in Eq. (118). 


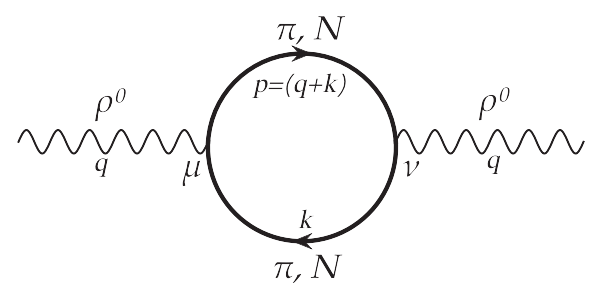

FIG. 1. Feynman diagram for the one-loop self-energy of the neutral $\rho$ meson.

\section{NUMERICAL RESULTS}

We begin this section by presenting the real and imaginary parts of the in-medium self-energy functions of $\rho^{0}$. As can be seen from Eqs. (89)-(115), we have only two nonzero form factors for the self-energy, which are $\Pi_{\alpha}$ and $\Pi_{\beta}$ for $q_{\perp}=0$. Let us first consider the zero magnetic field case for which the imaginary and real parts of $\Pi_{\alpha}$ and $\Pi_{\beta}$ are depicted in Figs. 2 and 3 respectively. In Fig. 2(a), $\operatorname{Im} \Pi_{\alpha}$ and $\operatorname{Im} \Pi_{\beta}$ due to the $\pi \pi$ loop are plotted as a function of invariant mass $\left(\sqrt{q^{2}}\right)$ of $\rho^{0}$ for vacuum as well as for medium $\left(T=160 \mathrm{MeV}\right.$ and $\left.\mu_{B}=400 \mathrm{MeV}\right)$ with $q_{z}=250 \mathrm{MeV}$. It is to be understood that in the case of vacuum the two form factors are equal. In this case, the only contribution comes from the unitary-I cut, which starts at $2 m_{\pi}$ in the invariant mass axis. With the increase in temperature, the degeneracy between the form factor gets lifted as well as their magnitudes are enhanced with respect to the vacuum. This is due to the enhancement of the thermal factor in Eq. (120), which increases the available phase space with the increase in temperature. The corresponding results for the NN loop is shown in Fig. 2(b) for which the threshold of the unitary-I cut is $2 m_{N}$. In this case, with the increase in temperature and density, the imaginary part decreases slightly with respect to the vacuum, which

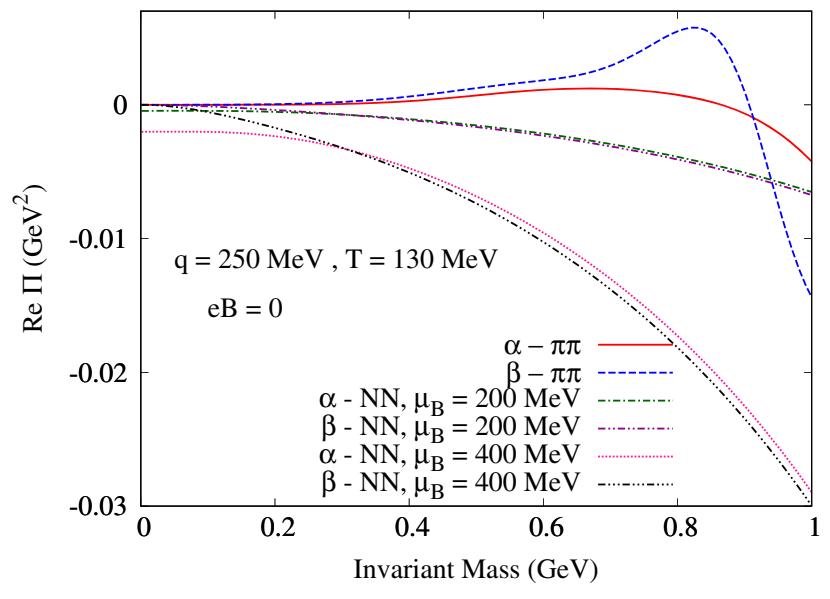

FIG. 3. Real part of the self-energy of $\rho^{0}$ as a function of invariant mass at zero magnetic field and at temperature $T=$ $130 \mathrm{MeV}$ with $\rho^{0}$ three-momentum $|\vec{q}|=250 \mathrm{MeV}$. The contributions from the NN (Nucleon-Nucleon) loop are shown for two different values of baryon chemical potential $\left(\mu_{B}=200\right.$ and $400 \mathrm{MeV})$.

can be understood from Eq. (124), where, because of the negative signs in front of the thermal distribution functions of the nucleons, the thermal factor reduces with the increase in temperature, thus showing opposite behavior as compared to the $\pi \pi$ loop.

In Fig. 3, $\operatorname{Re} \Pi_{\alpha}$ and $\operatorname{Re} \Pi_{\beta}$ are shown as a function of $\rho^{0}$ invariant mass at zero external magnetic field with $\rho^{0}$ longitudinal momentum $q_{z}=250 \mathrm{MeV}$ at temperature $T=130 \mathrm{MeV}$. For the $\pi \pi$ loop, the real part is positive at low invariant mass and becomes negative in the high invariant mass region in contrast to the NN loop for which the contribution to the real part is always negative. The real part due to the NN loop is shown for two different values of baryon chemical potential, $\mu_{B}=200$ and $400 \mathrm{MeV}$.

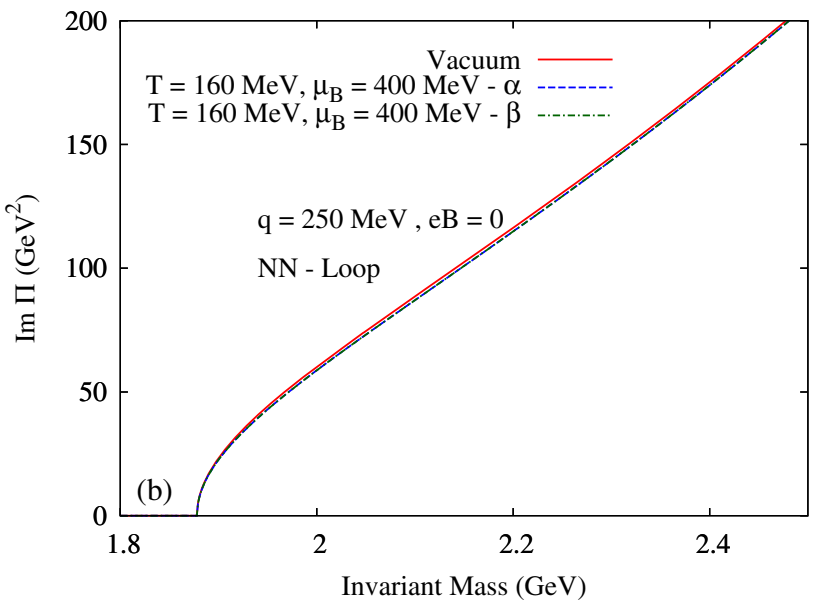

FIG. 2. Imaginary part of the self-energy of $\rho^{0}$ as a function of invariant mass at zero magnetic field and at $\rho^{0}$ three-momentum $|\vec{q}|=250 \mathrm{MeV}$. The vacuum self-energy for $T=\mu_{B}=0$ is compared with the in-medium one obtained at temperature $T=160 \mathrm{MeV}$ and baryon chemical potential $\mu_{B}=400 \mathrm{MeV}$ for the (a) $\pi \pi$ loop and (b) NN loop. 
For low values of $\mu_{B}$, the contribution of the NN loop is almost of the same order as the $\pi \pi$ loop; however, at high $\mu_{B}$, the contribution from the NN loop dominates over the $\pi \pi$ loop.

We now turn on the external magnetic field. For the check of consistency of the calculation at nonzero magnetic field, it is essential that $e B \rightarrow 0$ limit of nonzero magnetic field results reproduces the $e B=0$ one. In order to take the $e B \rightarrow 0$ limit numerically, we have considered up to 500 Landau levels for a convergent result. We have shown the imaginary part of the self-energy as a function of invariant mass of $\rho^{0}$ with longitudinal momentum $q_{z}=250 \mathrm{MeV}$ at temperature $T=130 \mathrm{MeV}$ and at baryon chemical potential $\mu_{B}=300 \mathrm{MeV}$ for the two cases: $e B=0$ and $e B \rightarrow 0$ in Fig. 4 separately for the $\pi \pi$ and NN loops. Figure 4(a) shows $\operatorname{Im} \Pi_{\alpha}$ for the $\pi \pi$ loop in which the $e B \rightarrow 0$ graph has a series of spikes infinitesimally separated from each other all over the whole invariant mass region, whereas the $e B=$ 0 graph is finite and well behaved. Interestingly, the $e B \rightarrow$ 0 graph does not miss the $e B=0$ curve, which implies that when average is done the $e B=0$ line will be exactly reproduced. The appearance of these spikes is due to the "threshold singularities" [31,38,39] at each Landau level, as can be understood from Eq. (132), where the Källén function goes to zero at each threshold of the unitary and Landau cuts defined in terms of the unit step functions therein, which is a consequence of the dimensional reduction. In order to extract physical and finite results out of these spikes, we have used Ehrenfest's coarse-graining (CG) $[38,40,41]$. In this method, the whole invariant mass region has been discretized in small bins followed by bin averages. In other words, the self-energy at a given $\sqrt{q_{\|}^{2}}$ is approximated by its average over the neighborhood around that point. This in turn smears out the spikelike structures. As can be seen in the figure, after $\mathrm{CG}, \operatorname{Im} \Pi_{\alpha}$ exactly matches with the analytic $e B=0$ graph. The corresponding comparison of the $e B \rightarrow 0$ and $e B=0$ result for $\operatorname{Im} \Pi_{\beta}$ due to the $\pi \pi$ loop is shown in Fig. 4(b). In this case, the
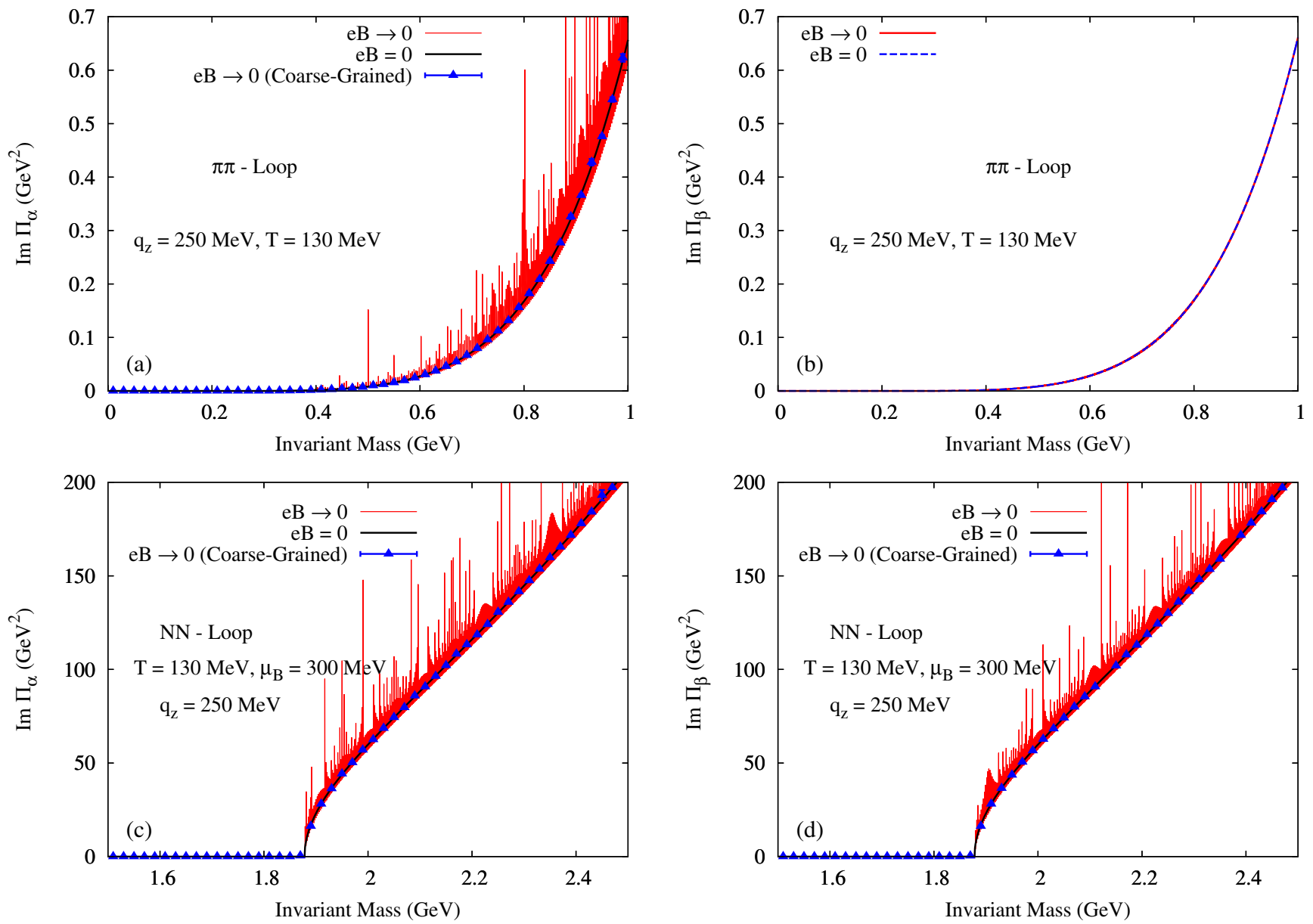

FIG. 4. The imaginary parts of the form factors as a function of the invariant mass at $e B=0$ have been compared with the imaginary parts at nonzero magnetic field in the numerical limit $e B \rightarrow 0$ at temperature $T=130 \mathrm{MeV}$ and at baryon chemical potential $\mu_{B}=$ $300 \mathrm{MeV}$ with $\rho^{0}$ longitudinal momentum $q_{z}=250 \mathrm{MeV}$. The contribution due the $\pi \pi$ loop from the form factors $\Pi_{\alpha}$ and $\Pi_{\beta}$ are shown in panels (a) and (b) respectively. The corresponding contributions due the NN loop are shown in panels (c) and (d). The respective CG quantities from the $e B \rightarrow 0$ results are also shown in (a), (c), and (d). 
$e B \rightarrow 0$ graph is finite and free from the threshold singularities, and it exactly matches the $e B=0$ graph. The absence of the threshold singularities in this case is due to an overall factor of Källén functions coming from $\tilde{\mathcal{N}}_{\pi, n l}^{00}$ in Eq. (133), which cancels the Källén functions in the denominator of Eq. (132). Thus, the $\operatorname{Im} \Pi_{\beta}$ due to the $\pi \pi$ loop does not require being coarse grained.

The corresponding results for the NN loop is depicted in Figs. 4(c) and 4(d). In this case, both the $\operatorname{Im}_{\alpha}$ and $\operatorname{Im} \Pi_{\beta}$ suffer threshold singularities, as there is no overall Källén functions coming from $\tilde{\mathcal{N}}_{\mathrm{p}, n l}^{\mu \nu}$. So, both the form factors have to be coarse grained, after which they exactly reproduce the $e B=0$ graphs.

We now turn our attention to the real part of the selfenergy at nonzero magnetic field and show how a numerical limit of $e B \rightarrow 0$ agrees with the $e B=0$ results. This has been shown in Fig. 5, where the real part of the form factors is shown as a function of $\rho^{0}$ invariant mass with longitudinal momentum $q_{z}=250 \mathrm{MeV}$ at temperature $T=$ $130 \mathrm{MeV}$ and at baryon chemical potential $\mu_{B}=$ $300 \mathrm{MeV}$ for the two cases $e B \rightarrow 0$ and $e B=0$. The contributions from the $\pi \pi$ and NN loops are shown separately. Figure 5(a) depicts $\operatorname{Re} \Pi_{\alpha}$, whereas Fig. 5(b) shows $\operatorname{Re} \Pi_{\beta}$. As can be seen from the figure, the $e B \rightarrow 0$ graphs exactly reproduce the $e B=0$ for the case of the NN loop, whereas, for the $\pi \pi$ loop, $e B \rightarrow 0$ is slightly deviated from the $e B=0$ graph but with an excellent qualitative agreement in their behavior with respect to the variation of the invariant mass of $\rho^{0}$. This small disagreement between the $e B \rightarrow 0$ and $e B=0$ graph is due to the inaccuracy in the numerical principal value integration of Eqs. (20) and (50) for which the two-particle bound state threshold $\sqrt{q_{\|}^{2}}>2 m_{\pi}=280 \mathrm{MeV}$ is less than the $\rho^{0}$ mass pole $m_{\rho}=0.770$ (in contrast, for the NN loop, the two-particle

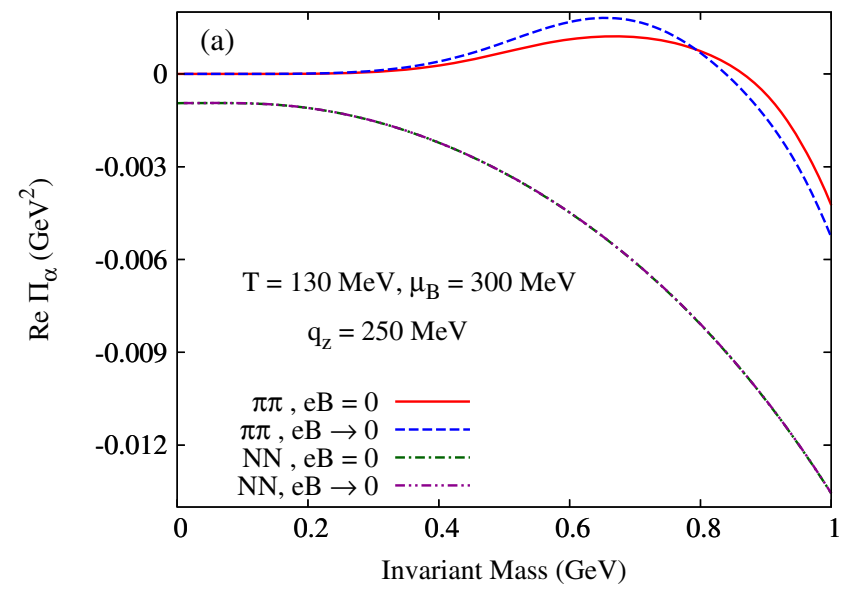

bound state threshold is at $\sqrt{q_{\|}^{2}}>2 m_{N}=1.878 \mathrm{GeV}$, much higher than the range of the plot).

Having checked the consistency of the nonzero magnetic field calculations, we now proceed to present the imaginary part of the self-energy for nonzero values of the magnetic field. In Fig. 6, the variation of $\operatorname{Im} \Pi_{\alpha}$ is shown as a function of $\rho^{0}$ invariant mass with longitudinal momentum $q_{z}=$ $250 \mathrm{MeV}$ at temperature $T=130 \mathrm{MeV}$ and at baryon chemical potential $\mu_{B}=300 \mathrm{MeV}$. We have plotted the self-energy up to $\sqrt{q_{\|}^{2}}=1.5 \mathrm{GeV}$ for which the unitary cut of the NN loop does not contribute. Figure 6(a) depicts $\operatorname{Im} \Pi_{\alpha}$ at magnetic field $e B=0.05 \mathrm{GeV}^{2}$, in which the spikes get separated from each other by a finite value and the form factor oscillates about the $e B=0$ graph. This is more clearly visible in the CG points, which are used to obtain a coarse-grained interpolated (CGI) graph. Figure 6 (b) shows the CGI imaginary parts at two different values of the magnetic field $\left(e B=0.05\right.$ and $\left.0.10 \mathrm{GeV}^{2}\right)$; both of them are found to oscillate about the $e B=0$ graph. Moreover, with the increase in magnetic field, the oscillation frequency decreases with an increase in the oscillation amplitude. This behavior of the imaginary part with increasing magnetic field is consistent with Fig. 4, where for the $e B \rightarrow 0$ case the oscillation frequency becomes infinite and the amplitude becomes zero, thus reproducing the $e B=0$ graph. Also, with the increase in magnetic field, the threshold of the unitary cut moves toward the higher invariant mass value, as discussed in Sec. VII. This has been shown clearly in the inset plot.

The corresponding results for the $\operatorname{Im} \Pi_{\beta}$ due to the $\pi \pi$ loop as a function of $\rho^{0}$ invariant mass with longitudinal momentum $q_{z}=250 \mathrm{MeV}$ at temperature $T=130 \mathrm{MeV}$ and at baryon chemical potential $\mu_{B}=300 \mathrm{MeV}$ are shown in Fig. 7 for the two different values of the magnetic field

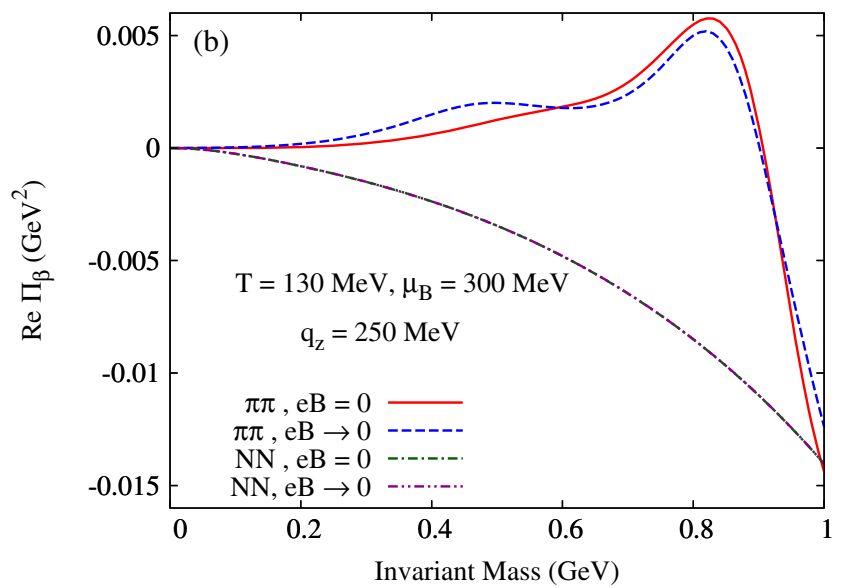

FIG. 5. The real part of the form factors as a function of the invariant mass at $e B=0$ have been compared with the real part at nonzero magnetic field in the numerical limit $e B \rightarrow 0$ at temperature $T=130 \mathrm{MeV}$ and at baryon chemical potential $\mu_{B}=300$ with $\rho^{0}$ longitudinal momentum $q_{z}=250 \mathrm{MeV}$. The contributions from the form factors (a) $\Pi_{\alpha}$ and (b) $\Pi_{\beta}$ are shown separately due to the $\pi \pi$ and NN loops. 

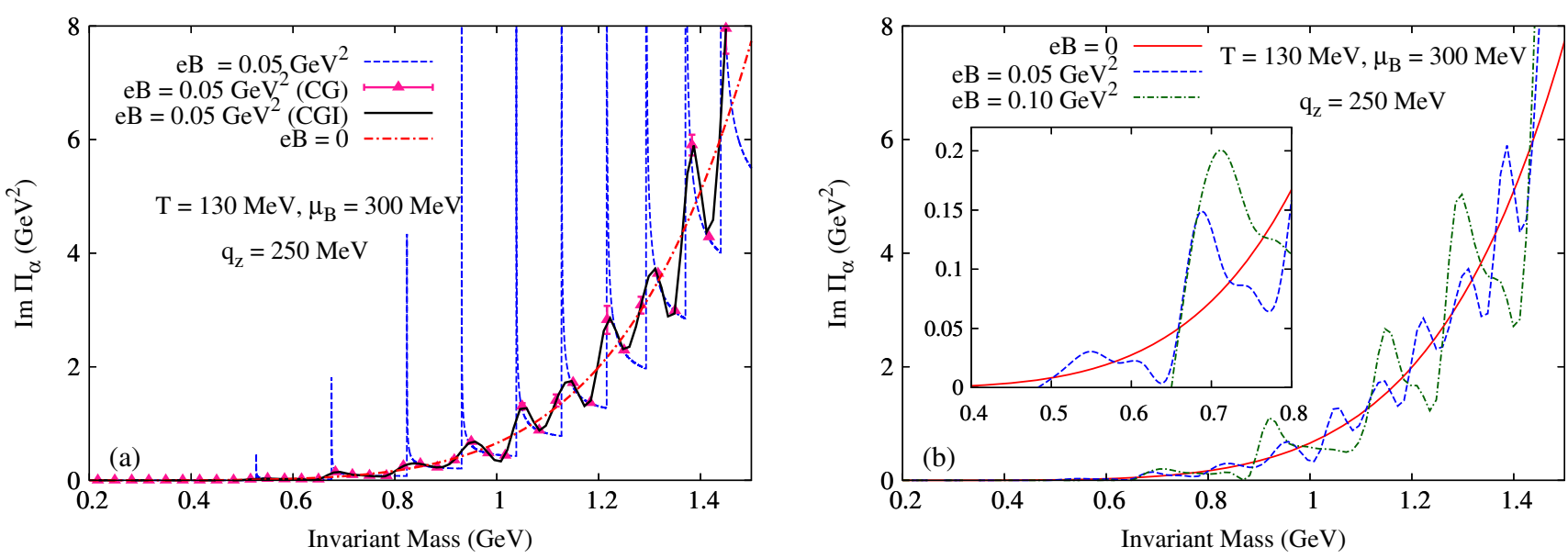

FIG. 6. The contribution from the form factor $\operatorname{Im} \Pi_{\alpha}$ to the imaginary part of the $\rho^{0}$ self-energy is shown as a function of invariant mass at temperature $T=130 \mathrm{MeV}$ and at baryon chemical potential $\mu_{B}=300$ with $\rho^{0}$ longitudinal momentum $q_{z}=250 \mathrm{MeV}$ for (a) two different values of magnetic field $\left(e B=0\right.$ and $\left.0.05 \mathrm{GeV}^{2}\right)$ and (b) three different values of magnetic field $(e B=0,0.05$, and $0.10 \mathrm{GeV}^{2}$ ). The $\mathrm{CG}$ as well as CGI results are shown in (a), whereas (b) shows only the CGI results. The inset plot in (b) shows the movement of the unitary cut threshold by focusing on a smaller range of invariant mass.

$e B=0.10$ and $0.20 \mathrm{GeV}^{2}$. Analogous to $\operatorname{Im} \Pi_{\alpha}, \operatorname{Im}_{\beta}$ also oscillates about the $e B=0$ curve, but in this case, the oscillation frequency is much smaller as compared to $\operatorname{Im} \Pi_{\alpha}$. The threshold of the unitary cut moves toward higher invariant mass with the increase in magnetic field as clearly depicted in the inset plot.

As discussed in Sec. VII, a nontrivial Landau cut contribution in the presence of external magnetic field may appear even if the loop particles have the same mass. In this case, we have observed Landau cut contribution only

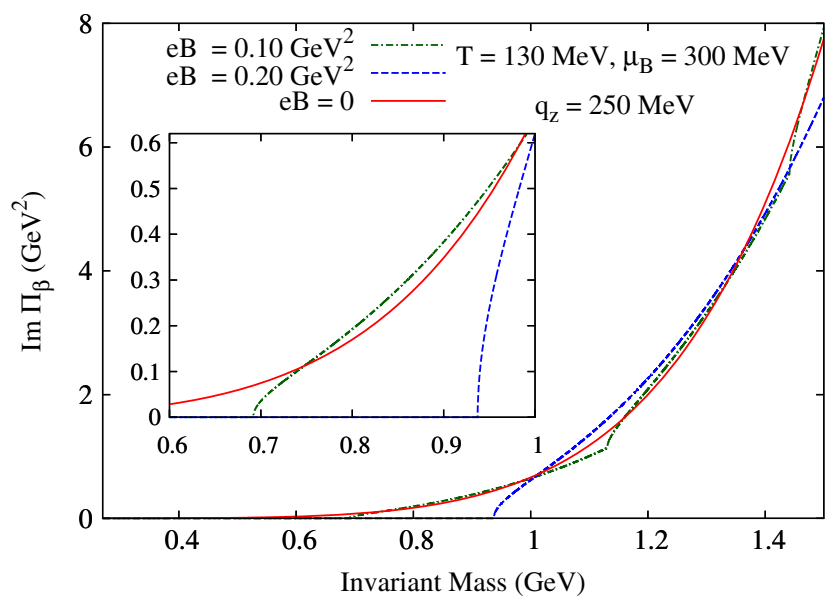

FIG. 7. The contribution from the form factor $\operatorname{Im}_{\beta}$ to the imaginary part of the $\rho^{0}$ self-energy is shown as a function of invariant mass at temperature $T=130 \mathrm{MeV}$ and at baryon chemical potential $\mu_{B}=300$ with $\rho^{0}$ longitudinal momentum $q_{z}=250 \mathrm{MeV}$ for three different values of magnetic field $\left(e B=0,0.05\right.$, and $\left.0.10 \mathrm{GeV}^{2}\right)$. The inset plot shows the movement of the unitary cut threshold by focusing on a smaller range of invariant mass. in $\operatorname{Im} \Pi_{\alpha}$, whereas the Landau cut does not appear in $\operatorname{Im} \Pi_{\beta}$. This can be understood from the expressions of the trace and 00 component of $\tilde{\mathcal{N}}_{\pi, n l}^{\mu \nu}$ and $\tilde{\mathcal{N}}_{\mathrm{p}, n l}^{\mu \nu}$ as given in Appendix F. It can be noticed that, for both the $\pi \pi$ and proton-proton loops, the expression for the trace (i.e., $\tilde{\mathcal{N}}_{\mu}^{\mu}$ ) contains two additional Kronecker delta functions $\delta_{l}^{n \pm 1}$ along with $\delta_{l}^{n}$, which is absent in the expressions for the 00 component (i.e., $\tilde{\mathcal{N}}^{00}$ ) [see Eqs. (F6)-(F9)]. This implies that, for $\operatorname{Im} \Pi_{\alpha}$, the loop particles can be in different Landau levels, whereas for $\operatorname{Im}_{\beta}$, the loop particles will always stay in the same Landau levels. Thus, as discussed in Sec. VII, the nontrivial Landau cuts will appear only in $\operatorname{Im}_{\alpha}$ and not in $\operatorname{Im}_{\beta}$. The contribution of the CGI Landau cuts to $\operatorname{Im} \Pi_{\alpha}$ as a function of $\rho^{0}$ invariant mass with longitudinal momentum $q_{z}=250 \mathrm{MeV}$ is shown in Fig. 8. It is to be noted that the Landau cuts also contain the threshold singularities and thus have to be coarse grained. Figure 8(a) shows the variation of $\operatorname{Im}_{\alpha}$ at temperature $T=130 \mathrm{MeV}$ and at baryon chemical potential $\mu_{B}=300 \mathrm{MeV}$ for three different values of the magnetic field $(e B=0.05,0.07$, and $0.10 \mathrm{GeV}^{2}$ ), whereas Fig. 8(b) shows the corresponding variation at magnetic field $\left(e B=0.10 \mathrm{GeV}^{2}\right)$ for two different values of temperature $(T=100$ and $130 \mathrm{MeV})$. The contributions due to the $\pi \pi$ loop and proton-proton loops are shown separately and in Fig. 8(b); the contribution due to the proton-proton loop is shown for two different values of baryon chemical potential $\left(\mu_{B}=200\right.$ and $300 \mathrm{MeV}$ ). As can be seen from the figures, the threshold of the Landau cuts due to the $\pi \pi$ loop is different (greater) than that of the proton-proton loop, which can be understood from the discussions of Sec. VII. The threshold for the $\pi \pi$ loop is $\sqrt{q_{\|}^{2}}<\left(\sqrt{m_{\pi}^{2}+e B}-\sqrt{m_{\pi}^{2}+3 e B}\right)$, 

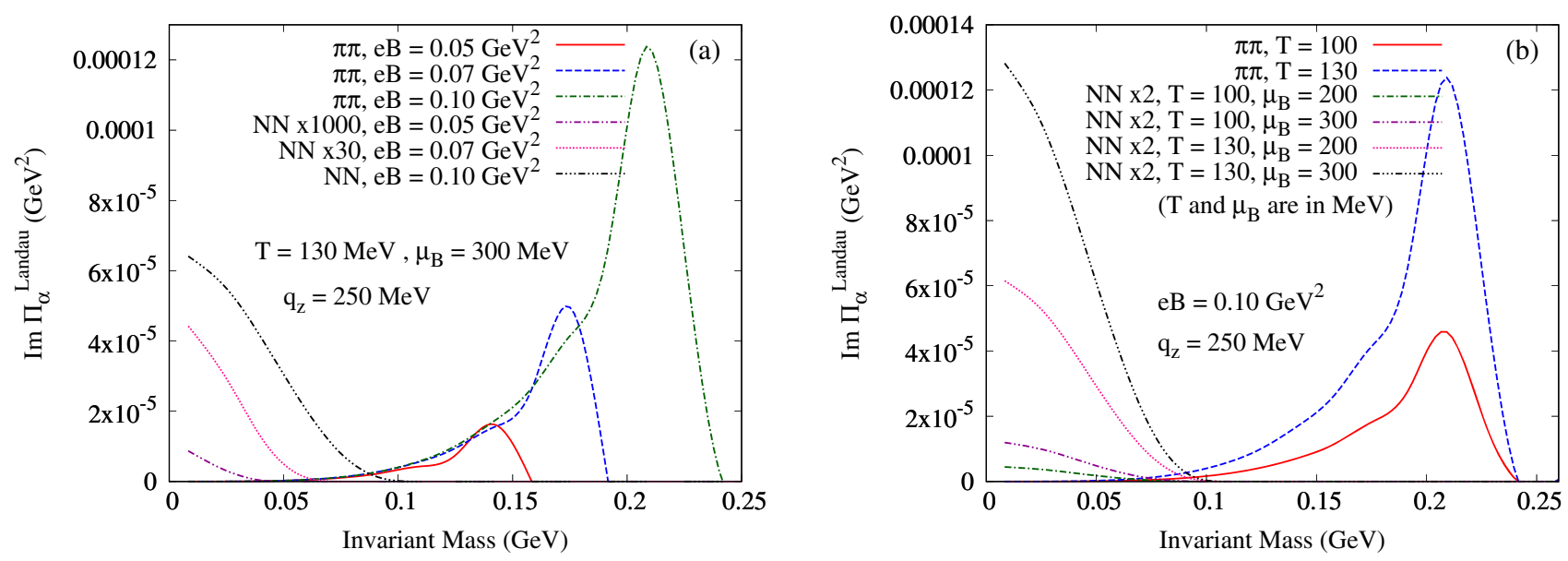

FIG. 8. The contribution from the form factor $\Pi_{\alpha}$ to the Landau cut of the CG imaginary part of the $\rho^{0}$ self-energy is shown as a function of invariant mass with $\rho^{0}$ longitudinal momentum $q_{z}=250 \mathrm{MeV}$ (a) at temperature $T=130 \mathrm{MeV}$ and at baryon chemical potential $\mu_{B}=300$ for three different values of magnetic field $\left(e B=0.05,0.07\right.$ and $\left.0.10 \mathrm{GeV}^{2}\right)$ and (b) at magnetic field $e B=$ $0.10 \mathrm{GeV}^{2}$ for two different values of temperature $(T=100$ and $130 \mathrm{MeV})$ and at baryon chemical potential $\left(\mu_{B}=200\right.$ and $\left.300 \mathrm{MeV}\right)$. The contributions from the $\pi \pi$ and NN loops are shown separately, and the latter is scaled with different factors for the sake of presentation.

whereas the same for the proton-proton loop is $\sqrt{q_{\|}^{2}}<$ $\left(m_{N}-\sqrt{m_{N}^{2}+2 e B}\right)$. The shift of the Landau cut threshold toward the higher invariant mass values with the increase in magnetic field can be clearly seen in Fig. 8(a). It is observed that the magnitude of the Landau cut contribution due to the proton-proton loop is much less than that of the $\pi \pi$ loop at lower values of the magnetic field, and they become comparable to each other only at $e B \gtrsim 0.10 \mathrm{GeV}^{2}$. In Fig. 8(a), we observe that with the increase in temperature and density the Landau cut contribution increases without changing its threshold in the invariant mass axis.

We now turn our attention to the real part of the selfenergy at finite temperature under external magnetic field. In Fig. 9, we show the thermal contribution to the real part of the self-energy as a function of invariant mass with $\rho^{0}$ longitudinal momentum $q_{z}=250 \mathrm{MeV}$ at temperature $T=130 \mathrm{MeV}$ and at baryon chemical potential $\mu_{B}=$ $300 \mathrm{MeV}$ for two different values of the magnetic field $\left(e B=0.05\right.$ and $\left.0.10 \mathrm{GeV}^{2}\right)$. The contributions from the $\pi \pi$ and NN loops are summed up in this figure. We notice that, with the increase in magnetic field, the thermal contribution to the real part of the self-energy oscillates about the $e B=$ 0 curve. The oscillation frequency decreases and the oscillation amplitude increases with the increase in magnetic field.

Next, in Fig. 10, the eB-dependent vacuum contribution to the real part of the self-energy is shown as a function of $\rho^{0}$ invariant mass with longitudinal momentum $q_{z}=$ $250 \mathrm{MeV}$ for two different values of magnetic field $\left(e B=0.10\right.$ and $\left.0.20 \mathrm{GeV}^{2}\right)$. Figures 10 (a) and 10(b) show the contributions from $\Pi_{\alpha}$ and $\Pi_{\beta}$ respectively. The contributions due to the $\pi \pi$ and proton-proton loops are shown separately. First of all, we note that at $e B=0$ these term will vanish. With the increase of the magnetic field, the eBdependent vacuum term also increases, and the contribution of $\Pi_{\beta}$ is more than $\Pi_{\alpha}$.

Having obtained the real and imaginary parts of the selfenergy, we now proceed to evaluate the in-medium spectral functions of $\rho^{0}$ under external magnetic field. We have from Eq. (106) the complete $\rho^{0}$ propagator as

$\overline{\bar{D}}^{\mu \nu}=A_{\alpha} P_{1}^{\mu \nu}+A_{\beta} P_{2}^{\mu \nu}+A_{\gamma} P_{3}^{\mu \nu}+A_{\delta} Q^{\mu \nu}+\xi q^{\mu} q^{\nu}$

where the coefficients are given in Eqs. (107)-(111) and the basis tensors are provided in Eqs. (72)-(75). Since we will

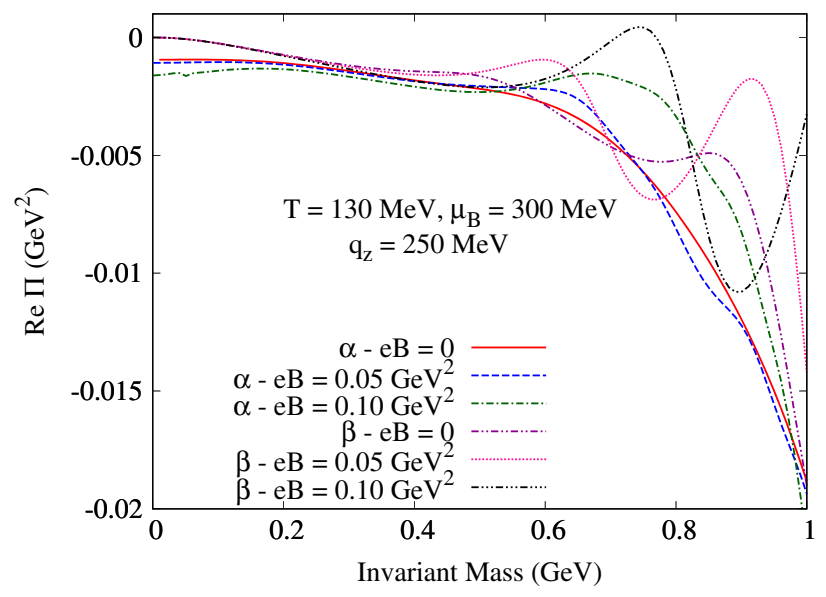

FIG. 9. The real part of the thermal self-energy of $\rho^{0}$ as a function of invariant mass at temperature $T=130 \mathrm{MeV}$ and at baryon chemical potential $\mu_{B}=300 \mathrm{MeV}$ with $\rho^{0}$ longitudinal momentum $q_{z}=250 \mathrm{MeV}$ is shown for three different values of magnetic field $\left(0,0.05\right.$, and $\left.0.10 \mathrm{GeV}^{2}\right)$. 

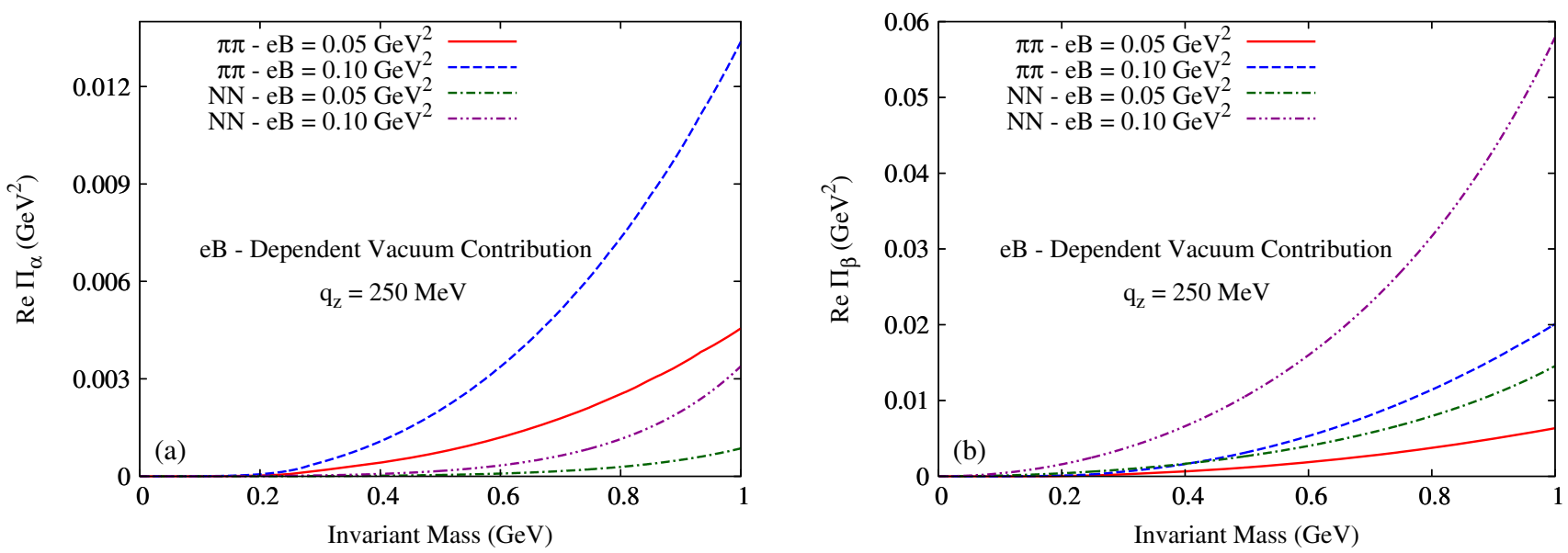

FIG. 10. The eB-dependent vacuum contribution to the real part of the self-energy of $\rho^{0}$ as a function of invariant mass with $\rho^{0}$ longitudinal momentum $q_{z}=250 \mathrm{MeV}$ is shown at two different values of magnetic field $\left(e B=0.05\right.$ and $\left.0.10 \mathrm{Gev}^{2}\right)$ for the form factors (a) $\Pi_{\alpha}$ and (b) $\Pi_{\beta}$. The contributions due to the $\pi \pi$ and proton-proton loops are shown separately.

be considering the special case $q_{\perp}=0$ for which $\Pi_{\alpha}=\Pi_{\gamma}$ and $\Pi_{\delta}=0$ as given in Eq. (112), the coefficients in the above equation become

$$
\begin{aligned}
A_{\alpha} & =\left(\frac{1}{q_{\|}^{2}-m_{\rho}^{2}+\Pi_{\alpha}}\right) \\
A_{\beta} & =\left(\frac{1}{q_{\|}^{2}-m_{\rho}^{2}+\Pi_{\beta}}\right) \\
A_{\gamma} & =\left(\frac{1}{q_{\|}^{2}-m_{\rho}^{2}+\Pi_{\gamma}}\right) \\
A_{\delta} & =0 \\
\xi & =\frac{-1}{q_{\|}^{2} m_{\rho}^{2}}
\end{aligned}
$$

so that the complete in-medium interacting propagator is given by

$$
\begin{aligned}
\overline{\bar{D}}^{\mu \nu}\left(q^{0}, q_{z}\right)= & \frac{P_{1}^{\mu \nu}}{\left(q_{\|}^{2}-m_{\rho}^{2}+\Pi_{\alpha}\right)}+\frac{P_{2}^{\mu \nu}}{\left(q_{\|}^{2}-m_{\rho}^{2}+\Pi_{\beta}\right)} \\
& +\frac{P_{3}^{\mu \nu}}{\left(q_{\|}^{2}-m_{\rho}^{2}+\Pi_{\alpha}\right)}-\frac{q_{\|}^{\mu} q_{\|}^{\nu}}{q_{\|}^{2} m_{\rho}^{2}} .
\end{aligned}
$$

It is clear from the above equation that there will be three modes for the propagation of the $\rho^{0}$ meson in the magnetized medium for the vanishing transverse momentum of $\rho^{0}$. Of the three modes, two are found to be degenerate (the first and third terms on the rhs of the above equation), leaving two distinct modes for the propagation of $\rho^{0}$, which we denote as mode-A and mode-B.
We now define the spectral function $S_{\rho}$ of $\rho^{0}$ for the two distinct modes as the imaginary part of the complete propagator, which is obtained from Eq. (148) as

$$
\begin{aligned}
S_{\rho}^{(A)} & =\operatorname{Im}\left[\frac{-1}{q_{\|}^{2}-m_{\rho}^{2}+\Pi_{\alpha}}\right] \\
& =\frac{\operatorname{Im} \Pi_{\alpha}}{\left(q_{\|}^{2}-m_{\rho}^{2}+\operatorname{Re} \Pi_{\alpha}\right)^{2}+\left(\operatorname{Im} \Pi_{\alpha}\right)^{2}}
\end{aligned}
$$

and

$$
\begin{aligned}
S_{\rho}^{(B)} & =\operatorname{Im}\left[\frac{-1}{q_{\|}^{2}-m_{\rho}^{2}+\Pi_{\beta}}\right] \\
& =\frac{\operatorname{Im} \Pi_{\beta}}{\left(q_{\|}^{2}-m_{\rho}^{2}+\operatorname{Re} \Pi_{\beta}\right)^{2}+\left(\operatorname{Im} \Pi_{\beta}\right)^{2}}
\end{aligned}
$$

In Fig. 11, the spectral function for the two modes at zero magnetic field is shown as a function of $\rho^{0}$ invariant mass with $\rho^{0}$ longitudinal momentum $q_{z}=250 \mathrm{MeV}$ at baryon chemical potential $\mu_{B}=300 \mathrm{MeV}$ for three different values of temperature $(T=100,130$, and $160 \mathrm{MeV})$. The vacuum spectral function (which is the same for the two modes) is also shown for comparison. We find that the spectral functions have a nice Breit-Wigner shape around the $\rho^{0}$ mass pole with a width $\mathcal{O}(150 \mathrm{MeV})$ corresponding to the decay of $\rho^{0} \rightarrow \pi^{+} \pi^{-}$. With the increase in temperature, the width of the spectral function increases, and the peak decreases. Physically, it corresponds to the enhancement of the decay process in the medium, implying that the $\rho^{0}$ become more unstable at a high temperature. It is important to note that, for the invariant mass region shown in the plot, the imaginary part of the self-energy that enters in the calculation of the spectral function is completely due to the unitary-I cut of the $\pi \pi$ loop. On the other hand, the 


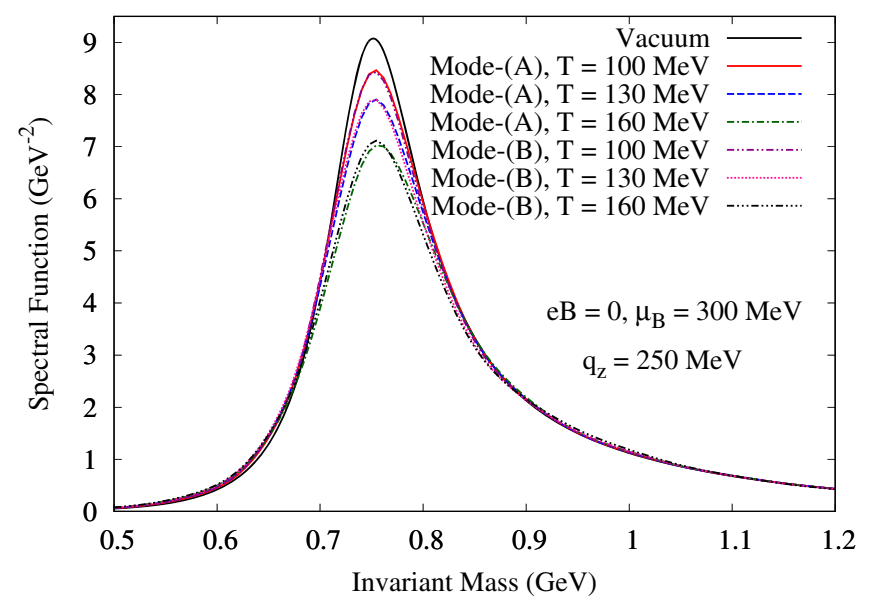

FIG. 11. The in-medium spectral function of $\rho^{0}$ as a function of invariant mass at zero magnetic field and at baryon chemical potential $\mu_{B}=300 \mathrm{MeV}$ with $\rho^{0}$ longitudinal momentum $q_{z}=$ $250 \mathrm{MeV}$ is shown for three different values of temperature $(T=100,130$, and $160 \mathrm{MeV})$ and for different modes. The vacuum spectral function is also shown for comparison.

real part of the self-energy that enters in the spectral function calculation has contributions from both the $\pi \pi$ and $\mathrm{NN}$ loops.

It can be noticed that, even at a higher temperature $(T \sim 160 \mathrm{MeV})$, the peak of the spectral functions have marginal shifts over the invariant mass axis which correspond to a negligible mass shift of the $\rho$ meson with respect to its vacuum mass. This is in agreement with the fact that, based on consideration of chiral symmetry alone, the mass of the $\rho$ meson does not change to $\mathcal{O}\left(T^{2}\right)$ [42]. At and above the critical temperature, chiral symmetry requires that the vector and axial-vector spectral function are identical [43] and is demonstrated in Ref. [44] using the sum rule approach. However, scenarios of the $\rho$ mass shift proposed by Brown and Rho [45] are also not ruled out, and the behavior of the $\rho$ meson mass can only indirectly be related to the chiral symmetry restoration. Though a significant shift of the $\rho$ mass has also been reported in Ref [46] using the Walecka model, the underlying phenomena behind this effect cannot be related to the partial restoration of chiral symmetry of QCD. Moreover, a majority of experiments does not find evidence for the mass shift of the $\rho$ meson in the medium, but rather a broadening of the spectral function is reported [47].

We now turn on the external magnetic field and show the spectral function of $\rho^{0}$ as a function of its invariant mass for the two modes in Fig. 12. The range of the invariant mass axis is taken as $0.5-1.2 \mathrm{GeV}$, which is dominated by the unitary cut contributions from the $\pi \pi$ loop. In Fig. 12(a), the spectral function with $\rho^{0}$ longitudinal momentum $q_{z}=$ $250 \mathrm{MeV}$ at temperature $T=130 \mathrm{MeV}$ and at baryon chemical potential $\mu_{B}=300 \mathrm{MeV}$ is shown for three different values of the magnetic field $(e B=0.10,0.15$, and $0.20 \mathrm{GeV}^{2}$ ). It is observed that, with the increase in the magnetic field, the two modes get well separated from each other and the threshold of the spectral function moves toward higher values of the invariant mass, corresponding to the magnetic field-dependent unitary cut threshold of the imaginary part of the self-energy. At sufficiently high values of the magnetic field, the spectral function misses the $\rho^{0}$ mass pole $(770 \mathrm{MeV})$ so that it loses its Breit-Wigner shape, which may be termed as $\rho^{0}$ "melting" in the presence of magnetic field. The critical value of the magnetic field for a given temperature and baryon chemical potential for which the $\rho^{0}$ will melt is discussed later.

In Fig. 12(b), the spectral function with $\rho^{0}$ longitudinal momentum $q_{z}=250 \mathrm{MeV}$ at magnetic field $e B=$ $0.10 \mathrm{GeV}^{2}$ and at a baryon chemical potential $\mu_{B}=$ $300 \mathrm{MeV}$ is shown for three different values of temperature $(T=100,130$, and $160 \mathrm{MeV})$. In this case, the threshold of the spectral function remains fixed, and for both the modes, the spectral function becomes shorter and wider with the increase in temperature with a marginal shift of its peak. The shift of the peak is due to the modification in the real part of the self-energy with the change in temperature.

Figure 12(c) depicts the spectral function with $\rho^{0}$ longitudinal momentum $q_{z}=250 \mathrm{MeV}$ at magnetic field $e B=0.10 \mathrm{GeV}^{2}$ and at temperature $T=160 \mathrm{MeV}$ for three different values of the baryon chemical potential $\left(\mu_{B}=200,300\right.$, and $\left.400 \mathrm{MeV}\right)$. Analogous to the previous case, the threshold of the spectral function remains fixed for both the modes. Since the baryon chemical potential only affects the real part of the self-energy in the given kinematic region, the peak of the spectral function changes its position (keeping the width almost the same) with the change in baryon chemical potential. It can be noticed that, in contrast to Fig. 12(b), the peak position of the spectral function is more sensitive to $\mu_{B}$ as compared to the temperature, which is due to the dominant contribution coming from the NN loop.

In Fig. 12(d), the spectral function at magnetic field $e B=0.10 \mathrm{GeV}^{2}$ and at temperature $T=130 \mathrm{MeV}$ with baryon chemical potential $\mu_{B}=300 \mathrm{MeV}$ is shown for two different values of $\rho^{0}$ longitudinal momentum $\left(q_{z}=0\right.$ and $500 \mathrm{MeV}$ ). In this case, the threshold of the spectral function remains the same, and the height of the spectral function increases with the increase of the longitudinal momentum.

We have already mentioned that a nontrivial Landau cut in the physical kinematic region would appear in the presence of the external magnetic field. In our case, the nonzero contribution to the Landau cut comes only from the form factor $\operatorname{Im} \Pi_{\alpha}$, which is reflected in the spectral function of mode-A. In Fig. 13, the spectral function as a function of $\rho^{0}$ invariant mass with $\rho^{0}$ longitudinal momentum $q_{z}=250 \mathrm{MeV}$ is shown in the low invariant mass region, which is dominated by the Landau cut contribution. It can be observed that the magnitude of the spectral 

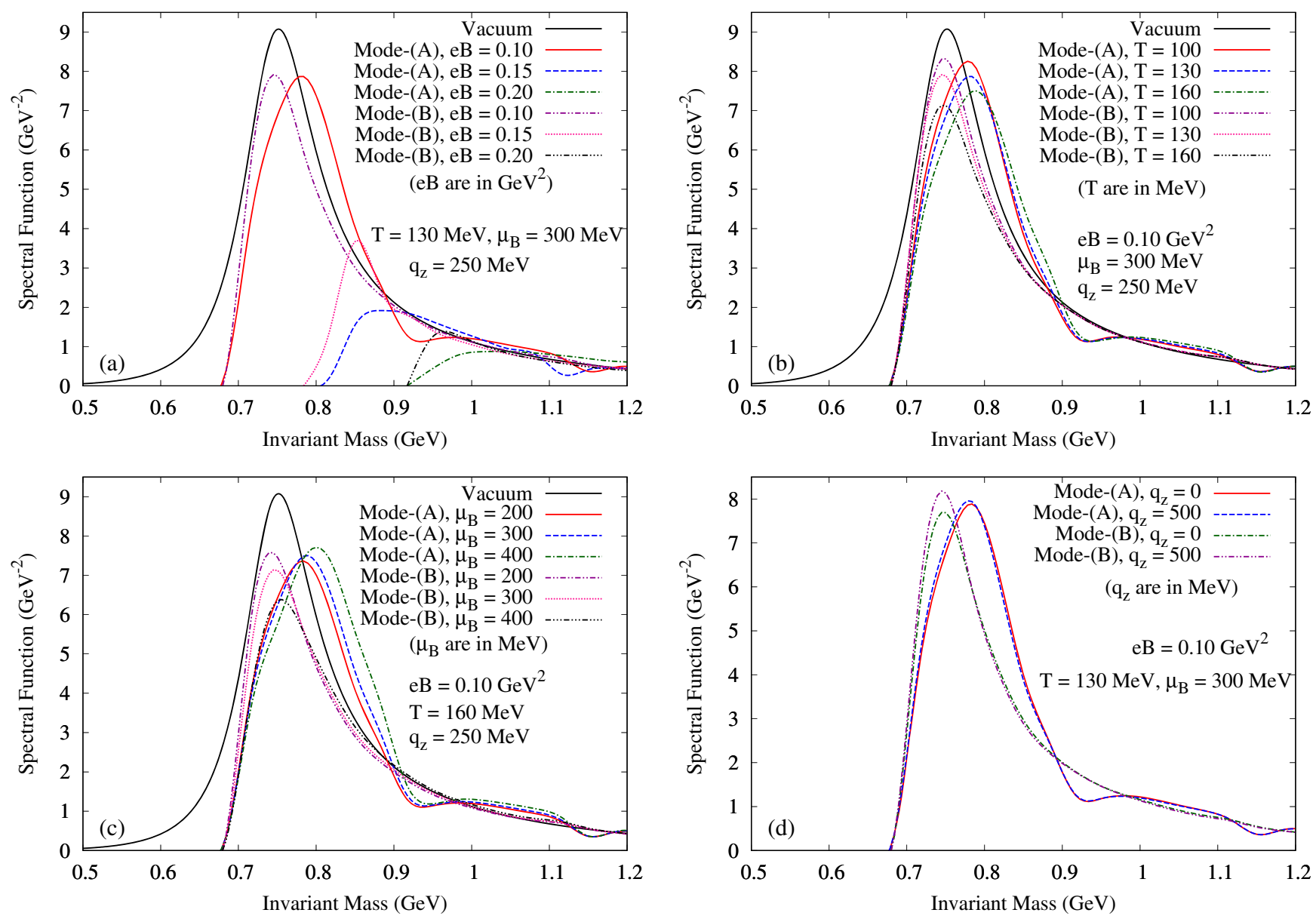

FIG. 12. The in-medium spectral functions of $\rho^{0}$ as a function of invariant mass are shown for different modes (a) at temperature $T=130 \mathrm{MeV}$ and at baryon chemical potential $\mu_{B}=300 \mathrm{MeV}$ with $\rho^{0}$ longitudinal momentum $q_{z}=250 \mathrm{MeV}$ for three different values of magnetic field $\left(e B=0.10,0.15\right.$ and $\left.0.20 \mathrm{GeV}^{2}\right)$, (b) at magnetic field $e B=0.10 \mathrm{GeV}^{2}$ and at baryon chemical potential $\mu_{B}=300 \mathrm{MeV}$ with $\rho^{0}$ longitudinal momentum $q_{z}=250 \mathrm{MeV}$ for three different values of temperature $(T=100,130$, and $160 \mathrm{MeV})$, (c) at magnetic field $e B=0.10 \mathrm{GeV}^{2}$ and at temperature $T=160 \mathrm{MeV}$ with $\rho^{0}$ longitudinal momentum $q_{z}=250 \mathrm{MeV}$ for three different values of baryon chemical potential $\left(\mu_{B}=200,300\right.$, and $\left.400 \mathrm{MeV}\right)$, and (d) at magnetic field $e B=0.10 \mathrm{GeV}^{2}$ and at temperature $T=130 \mathrm{MeV}$ with baryon chemical potential $\mu_{B}=300 \mathrm{MeV}$ for two different values of $\rho^{0}$ longitudinal momentum $\left(q_{z}=0\right.$ and $\left.500 \mathrm{MeV}\right)$. The vacuum spectral function is also shown for comparison.

function in this region is much lower as compared to the unitary cut regions. Figure 13(a) shows the spectral function at temperature $T=130 \mathrm{MeV}$ and at baryon chemical potential $\mu_{B}=300 \mathrm{MeV}$ for three different values of magnetic field ( $e B=0.10,0.15$, and $0.20 \mathrm{GeV}^{2}$ ). As can be seen in the graph, the threshold of the Landau cut moves toward the higher values of invariant mass with the increase in magnetic field as a consequence of similar behavior of the Landau cut, as shown in Fig. 8. Also, the height of the spectral function is enhanced with the increase in eB. Figure 13(b) shows the corresponding plots of the spectral function at magnetic field $e B=0.10 \mathrm{GeV}^{2}$ for four different combinations of temperature and baryon chemical potential $\left[\left(T=100 \mathrm{MeV}, \mu_{B}=300 \mathrm{MeV}\right),(T=\right.$ $\left.130 \mathrm{MeV}, \quad \mu_{B}=300 \mathrm{MeV}\right), \quad\left(T=160 \mathrm{MeV}, \quad \mu_{B}=\right.$ $300 \mathrm{MeV})$, and $\left.\left(T=160 \mathrm{MeV}, \mu_{B}=400 \mathrm{MeV}\right)\right]$. As can be seen in the graph, the height of the spectral function increases with the increase in temperature and density, owing to an enhancement of the corresponding scattering processes in the presence of external magnetic field.

We now proceed to obtain the effective mass and dispersion relation of the $\rho^{0}$ in a magnetized medium. They follow from the pole of the complete $\rho^{0}$ propagator given in Eq. (148), which are obtained by solving the following transcendental equations,

$$
\begin{aligned}
& \omega^{2}-q_{z}^{2}-m_{\rho}^{2}+\operatorname{Re}_{\alpha}\left(q^{0}=\omega, q_{z}, e B, T, \mu_{B}\right)=0 \\
& \omega^{2}-q_{z}^{2}-m_{\rho}^{2}+\operatorname{Re}_{\beta}\left(q^{0}=\omega, q_{z}, e B, T, \mu_{B}\right)=0,
\end{aligned}
$$

the numerical solutions $\omega=\omega\left(q_{z}, e B, T, \mu_{B}\right)$ of which represent the dispersion relations for the mode- $A$ and mode-B corresponding to $\rho^{0}$ propagation in the magnetized medium. The effective mass $m_{\rho}^{*}$ of $\rho^{0}$ is obtained from the 

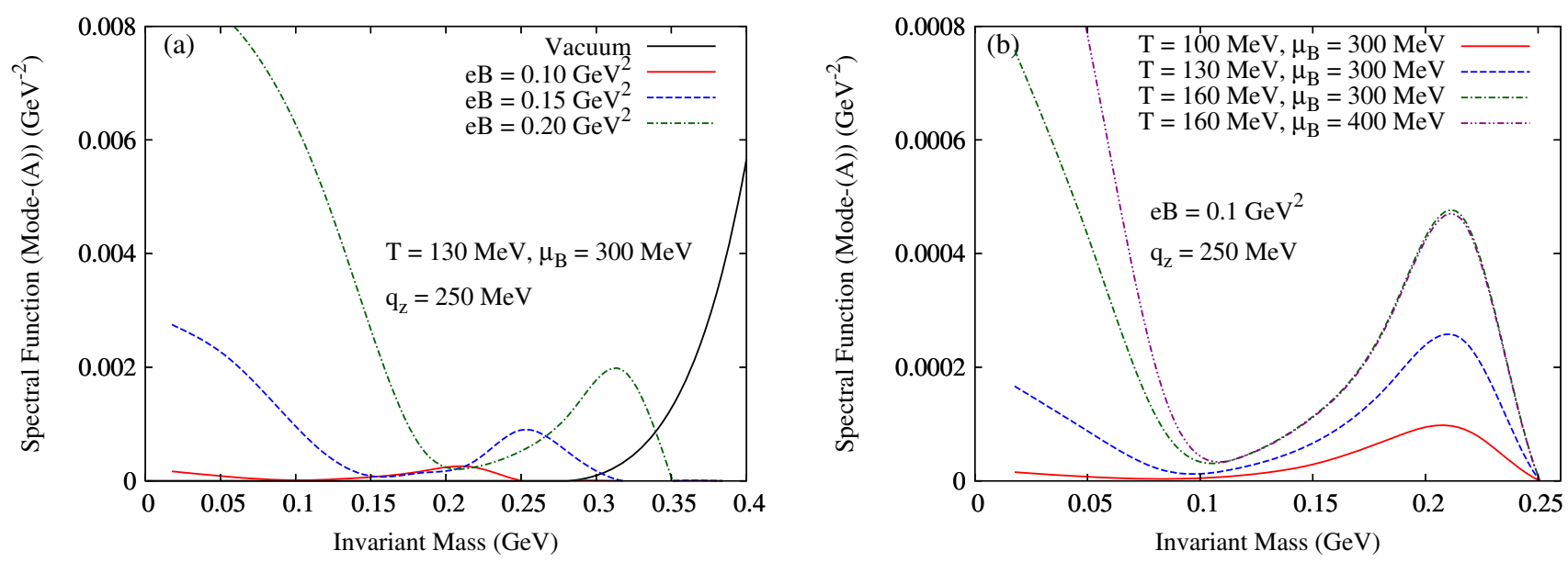

FIG. 13. The in-medium spectral functions of $\rho^{0}$ for mode-A as a function of invariant mass is shown in the low invariant mass region dominated by Landau cut contributions with $\rho^{0}$ longitudinal momentum $q_{z}=250 \mathrm{MeV}$ : (a) At temperature $T=130 \mathrm{MeV}$ and at baryon chemical potential $\mu_{B}=300 \mathrm{MeV}$ for three different values of magnetic field $\left(e B=0.10,0.15\right.$, and $\left.0.20 \mathrm{GeV}^{2}\right)$ and $(\mathrm{b})$ at magnetic field $e B=0.10 \mathrm{GeV}^{2}$ for four different combinations of temperature and baryon chemical potential $[(T=100 \mathrm{MeV}$, $\left.\mu_{B}=300 \mathrm{MeV}\right),\left(T=130 \mathrm{MeV}, \mu_{B}=300 \mathrm{MeV}\right),\left(T=160 \mathrm{MeV}, \mu_{B}=300 \mathrm{MeV}\right)$, and $\left.\left(T=160 \mathrm{MeV}, \mu_{B}=400 \mathrm{MeV}\right)\right]$.

dispersion relation by setting $q_{z}=0$, i.e., $m_{\rho}^{*}\left(e B, T, \mu_{B}\right)=$ $\omega\left(q_{z}=0, e B, T, \mu_{B}\right)$.

Figure 14(a) depicts the variation of $m_{\rho}^{*} / m_{\rho}$ as a function of magnetic field at temperature $T=130 \mathrm{MeV}$ and at baryon chemical potential $\mu_{B}=300 \mathrm{MeV}$. The effective mass for the two modes starts from the same value around $e B=0$, and with the increase in magnetic field, they get separated. For both modes, the effective $\rho^{0}$ mass decreases with the increase in the magnetic field, which is due to the strong positive contribution coming from the dominating eB-dependent vacuum part. The effect of the magnetic field is found to be more in mode-B as compared to mode-A. At a magnetic field value $e B=0.20 \mathrm{GeV}^{2}$, the effective $\rho^{0}$ mass in mode-A decreases by about $2 \%$, whereas for mode-B, it decreases by about $10 \%$. Figure 14(b) depicts the corresponding variation of effective mass with temperature at magnetic field $e B=0.10 \mathrm{GeV}^{2}$ and at baryon chemical potential $\mu_{B}=300 \mathrm{MeV}$. We find that, for both modes, the effective mass of $\rho^{0}$ gets enhanced by a small amount with the increase in temperature. Even at $T=160 \mathrm{MeV}$, the change in effective mass is less than $2 \%$. In Fig. 14(c), the variation of the effective $\rho^{0}$ mass is shown as a function of baryon chemical potential at a magnetic field $e B=0.10 \mathrm{GeV}^{2}$ and at temperature $T=130 \mathrm{MeV}$. In this case also, we observe an enhancement of the effective mass for both the modes with the increase in baryon density. Though the effect of $\mu_{B}$ on effective mass is more at a higher value of $\mu_{B}$, the change in the effective mass remains less than $2 \%$ even at $\mu_{B}=500 \mathrm{MeV}$.

Next, we present the dispersion curves of $\rho^{0}$ propagation in the magnetized medium for both the modes in Fig. 15. We have plotted the energy $\omega$ of the $\rho^{0}$ scaled with the inverse of the vacuum $\rho$ mass $m_{\rho}=770 \mathrm{MeV}$ as a function of the longitudinal momentum of $\rho^{0}$. Figure 15(a) depicts the dispersion curves at temperature $T=130 \mathrm{MeV}$ and at baryon chemical potential $\mu_{B}=300 \mathrm{MeV}$ for two different values of magnetic field $(e B=0.10$ and $0.20 \mathrm{GeV}^{2}$ ). Figure 15 (b) shows the same at magnetic field $e B=0.10 \mathrm{GeV}^{2}$ and baryon chemical potential $\mu_{B}=$ $300 \mathrm{MeV}$ for two different temperatures $(T=100$ and $160 \mathrm{MeV}$ ). Finally, Fig. 15(c) shows the corresponding graphs at magnetic field $e B=0.10 \mathrm{GeV}^{2}$ and at temperature $T=130 \mathrm{MeV}$ for two different values of baryon chemical potential $\left(\mu_{B}=200\right.$ and $\left.400 \mathrm{MeV}\right)$. In all the cases, the dispersion curves are well separated from each other at lower transverse momentum. With the increase in $q_{z}$, the loop correction becomes subleading with respect to the kinetic energy of $\rho^{0}$, and thus it approaches a lightlike dispersion.

Finally, we calculate the decay width of $\rho^{0}$ for the decay into charged pions, which is defined for the two modes as

$\Gamma^{(A)}\left(e B, T, \mu_{B}\right)=\frac{\operatorname{Im}_{\alpha}\left(q^{0}=m_{\rho}^{*}, q_{z}=0, e B, T, \mu_{B}\right)}{m_{\rho}^{*}\left(e B, T, \mu_{B}\right)}$

$\Gamma^{(B)}\left(e B, T, \mu_{B}\right)=\frac{\operatorname{Im} \prod_{\beta}\left(q^{0}=m_{\rho}^{*}, q_{z}=0, e B, T, \mu_{B}\right)}{m_{\rho}^{*}\left(e B, T, \mu_{B}\right)}$.

In Fig. 16, the variation of the decay width $\Gamma$ of $\rho^{0}$ scaled with the inverse of its vacuum width $\left(\Gamma_{0}=156 \mathrm{MeV}\right)$ for the two modes is shown as a function of magnetic field. Note that the vacuum decay width is obtained from the imaginary part of the vacuum self-energy as 

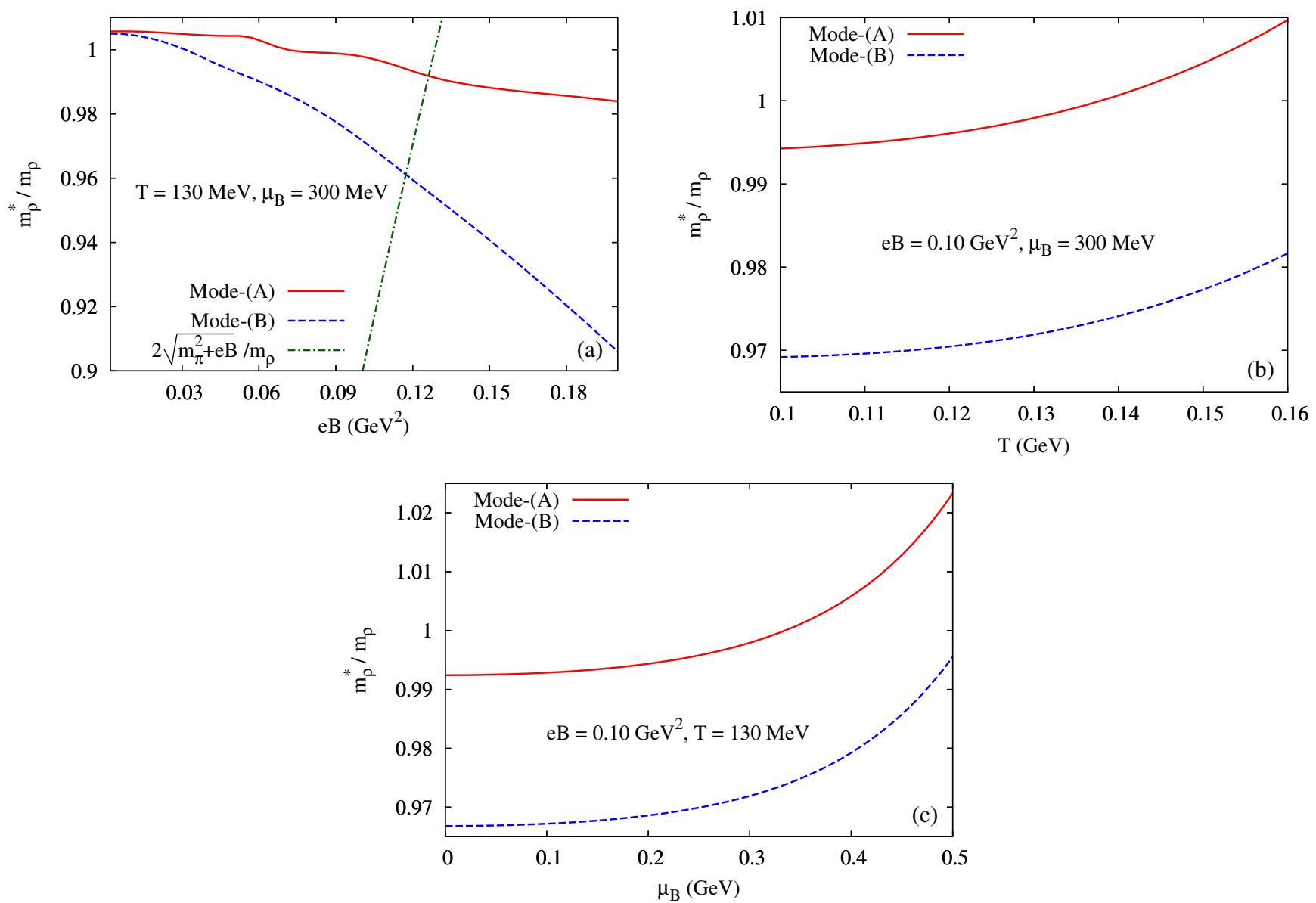

FIG. 14. The ratio of effective mass of $\rho^{0}$ to its vacuum mass for different modes (a) as a function of magnetic field at temperature $T=130 \mathrm{MeV}$ and at baryon chemical potential $\mu_{B}=300 \mathrm{MeV}$, (b) as a function of temperature at magnetic field $e B=0.10 \mathrm{GeV}^{2}$ and at baryon chemical potential $\mu_{B}=300 \mathrm{MeV}$, and (c) as a function of baryon chemical potential at temperature $T=130 \mathrm{MeV}$ and at magnetic field $e B=0.10 \mathrm{GeV}^{2}$. The green dash-dotted curve in (a) corresponds to the unitary cut threshold for decay of $\rho^{0} \rightarrow \pi^{+} \pi^{-}$. Here, $m_{\rho}=770 \mathrm{MeV}$.

$$
\Gamma_{0}=\frac{\operatorname{Im} \Pi_{\text {pure-vac }}\left(q^{0}=m_{\rho}, \vec{q}=\overrightarrow{0}\right)}{m_{\rho}}=156 \mathrm{MeV} .
$$

Results are presented for two different combinations of temperature and baryon chemical potential $[(T=130 \mathrm{MeV}$, $\left.\mu_{B}=300 \mathrm{MeV}\right)$ and $\left.\left(T=160 \mathrm{MeV}, \mu_{B}=400 \mathrm{MeV}\right)\right]$. Because of the presence of threshold singularity in $\operatorname{Im} \Pi_{\alpha}, \Gamma^{(A)}$ also suffers from the presence of threshold singularity for which it needs to be coarse grained. However, $\operatorname{Im} \Pi_{\beta}$ and hence $\Gamma^{(B)}$ are finite and free from the singularities. As can be seen from the figure, the ratio $\Gamma / \Gamma_{0}$ starts from a value greater than unity near $e B=0$, which is due to the enhancement of the decay width over its vacuum value due to the effect of finite temperature and density. Also, for a particular value of magnetic field, the larger decay width is observed at higher temperature and density. Near $e B=0$, the two modes have almost the same decay widths, which begin to differ from each other with the increase in the magnetic field. An oscillatory behavior of the decay width can be clearly seen throughout the magnetic field range. One should also notice that, for both modes, the oscillation amplitude increases, whereas oscillation frequency decreases with eB. Finally, at a critical value of the magnetic field, the decay width becomes zero. This is because of fact that the eB-dependent unitary cut threshold for the $\pi \pi$ loop has to satisfy

$$
m_{\rho}^{*}(e B)>2 \sqrt{m_{\pi}^{2}+e B}
$$

for a kinematically favorable decay of $\rho^{0} \rightarrow \pi^{+} \pi^{-}$. But with the increase in magnetic field, the rhs of the above equation increases, whereas $m_{\rho}^{*}$ on the lhs decreases so that at some critical value of magnetic field the above inequality is violated and the decay width becomes zero. Physically, it means that $\rho^{0}$ becomes stable against the decay into the $\pi^{+} \pi^{-}$pair. This critical value of the field may be considered the critical value of the magnetic field required for the melting of the spectral function of $\rho^{0}$. 

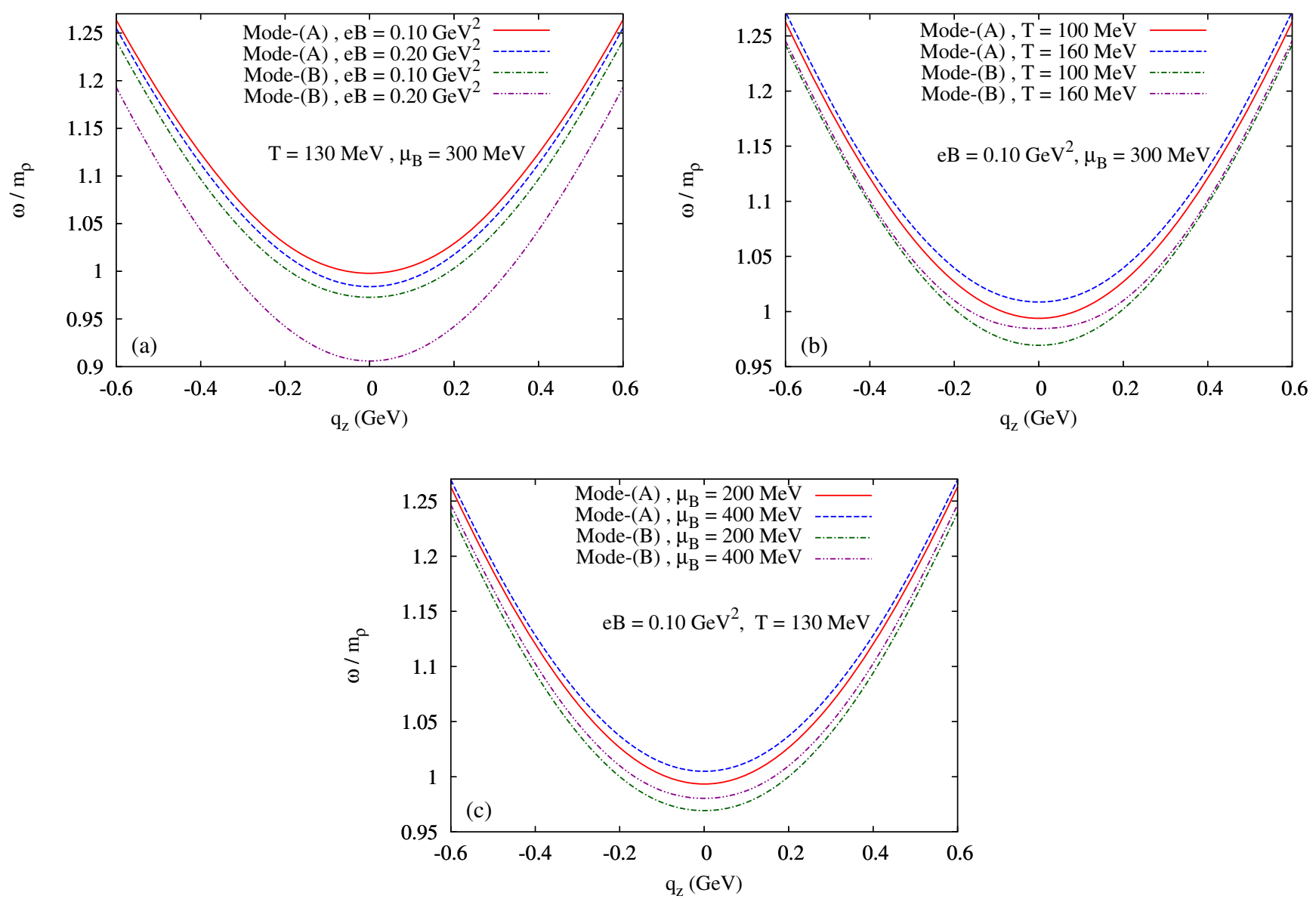

FIG. 15. The dispersion relations of $\rho^{0}$ for different modes: (a) At temperature $T=130 \mathrm{MeV}$ and at baryon chemical potential $\mu_{B}=300 \mathrm{MeV}$ for two different values of magnetic field $\left(e B=0.10\right.$ and $\left.0.20 \mathrm{GeV}^{2}\right)$, (b) at magnetic field $e B=0.10 \mathrm{GeV}^{2}$ and at baryon chemical potential $\mu_{B}=300 \mathrm{MeV}$ for two different temperatures $(T=100$ and $160 \mathrm{MeV})$, and (c) at magnetic field $e B=$ $0.10 \mathrm{GeV}^{2}$ and at temperature $T=130 \mathrm{MeV}$ for two different values of baryon chemical potential $\left(\mu_{B}=200\right.$ and $\left.400 \mathrm{MeV}\right)$.

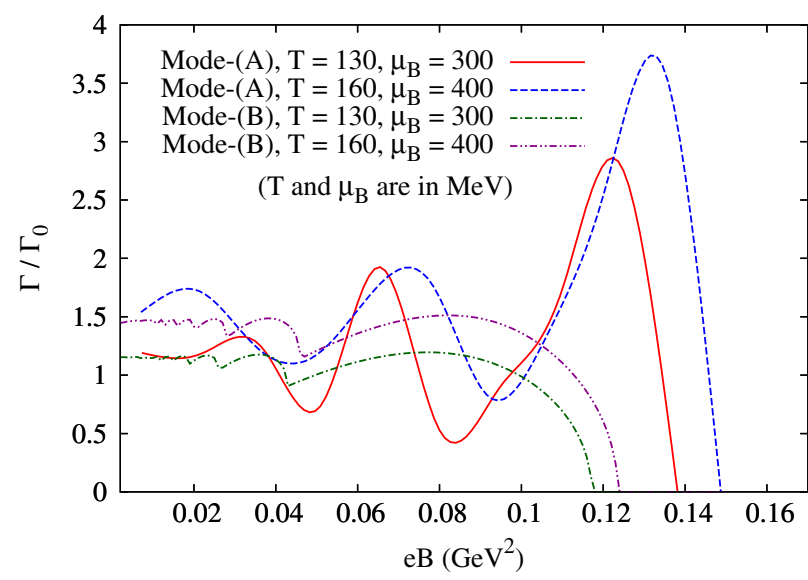

FIG. 16. The ratio of the decay width of $\rho^{0}$ to its vacuum width as a function of magnetic field for different modes with two different combinations of temperature and baryon chemical potential $\left[\left(T=130 \mathrm{MeV}, \mu_{B}=300 \mathrm{MeV}\right)\right.$ and $(T=160 \mathrm{MeV}$, $\left.\left.\mu_{B}=400 \mathrm{MeV}\right)\right]$. Here, $\Gamma_{0}=156 \mathrm{MeV}$.
In order to calculate the critical value of the magnetic field $e B_{c}$ for a given temperature $T$ and baryon chemical potential $\mu_{B}$, we need to solve the transcendental equation

$$
m_{\rho}^{*}\left(e B_{c}, T, \mu_{B}\right)=2 \sqrt{m_{\pi}^{2}+e B_{c}} .
$$

The green dash-dotted curve in Fig. 14(a) corresponds to $m_{\rho}^{*} / m_{\rho}=2 \sqrt{m_{\pi}^{2}+e B}$ so that the intersection of this curve with the $m_{\rho}^{*}=m_{\rho}^{*}(e B)$ represents the solution of the above equation. In Fig. 17, we show the variation of the critical magnetic field $e B_{c}$ for the two decay modes. Figure 17(a) depicts $e B_{c}$ as a function of temperature for two different values of baryon chemical potential $\left(\mu_{B}=50\right.$ and $200 \mathrm{MeV}$ ), whereas Fig. 17(b) shows the corresponding variation with baryon chemical potential at two different values of temperature $(T=100$ and $160 \mathrm{MeV})$. Although with fixed temperature the variation with respect to $\mu_{B}$ shows a monotonically increasing trend, both plots suggest nonmonotonic variations of the critical magnetic field with respect to the temperature. More specifically, there exists a 

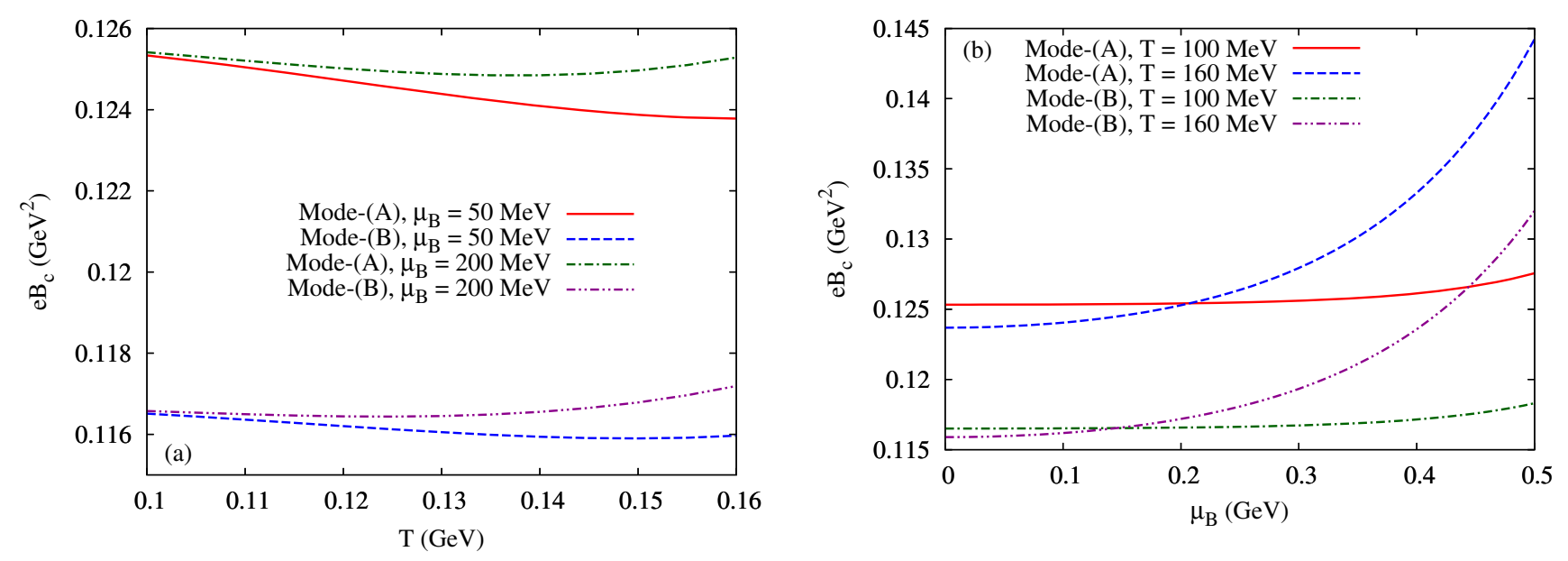

FIG. 17. The variation of the critical value of magnetic field for stopping the decay of $\rho^{0}$ into the $\pi^{+} \pi^{-}$pair for different modes as a function of (a) temperature at two different values of baryon chemical potential $\left(\mu_{B}=50\right.$ and $\left.200 \mathrm{MeV}\right)$ and (b) baryon chemical potential at two different values of temperature $(T=100$ and $160 \mathrm{MeV})$.

maximum value of chemical potential [see Fig. 17(b)] below which the critical field decreases with the temperature there by requiring a relatively weaker magnetic field to completely stop the particular decay channel. However, for even larger values of $\mu_{B}$, a significant increase with temperature can be observed for both of the decay modes.

A few comments on the magnitude of the external magnetic field are in order. The analytical expressions provided in this paper are valid for any arbitrary value of the external magnetic field which is constant in space-time. In presenting numerical results, we have considered magnetic field values in the range $0 \leq e B \leq 0.20 \mathrm{GeV}^{2}$. It is worth noting that the magnetic field created in the HIC experiments is expected to decay rapidly with time [48]. However, a nonzero electrical conductivity of the strongly interacting fireball could possibly sustain the external magnetic field a bit longer [49-51], implying a slowly varying function of time during the entire lifetime of the Quark-Gluon-Plasma. The magnitude of the external magnetic field at the time of chemical freeze-out (when the hadronic degrees of freedom manifest) is expected to be small because of the very small conductivity of the hadron gas. The experimental estimation of the same is not reported yet. In order to understand the plasma properties from the experimental data, one solves the relativistic magnetohydrodynamics equation usually with the assumption of ideal Quark-Gluon-Plasma fluid in the background electromagnetic field [52-54]. However, the ideal fluidity assumption can only be validated after knowing the transport coefficients at temperatures of phenomenological interest which are not yet certain. Despite these uncertainties, it should be mentioned here that the complete blocking of the neutral $\rho$ decay seems to be quite unlikely in the recent energy regimes of the HIC experiments. Although, one might expect a suppression in the $\rho^{0} \rightarrow \pi^{+} \pi^{-}$channel. Being the only possible strong decay channel of the $\rho^{0}$ meson, its suppression is expected to lead to the enhancement of dilepton and photon productions from $\rho^{0}$ decay. For example, the $\rho^{0} \rightarrow \pi^{0} \gamma$ channel is expected to possess a $64 \%$ branching ratio at the critical magnetic field of the order $10^{15} \mathrm{~T}$ [55]. However, recent measurement [56,57] shows almost no suppression in the strong decay channel of $\rho^{0}$ in peripheral $\mathrm{Pb}-\mathrm{Pb}$ collisions (case of nonzero external magnetic field) at LHC energies. However, the observed suppression in the central region (case of zero external magnetic field) is interpreted as the rescattering mechanism in the hadronic medium. Thus, this suggests that the magnetic effects on the neutral $\rho$ decay, if they exist, are negligibly small in the current HIC scenario. On the other hand, such magnetic modifications of mesonic properties can occur in situations present inside the high density compact objects with strong magnetic field such as magnetars. The tools used in the present work can be used to see the effects of the changes of hadronic properties on the equation of state, symmetry energy, mass-radius relationship, etc., after generalization to models appropriate for the description of hadronic matter at low temperature and at high density supposed to be present in a magnetized neutron star or magnetar $[58,59]$.

\section{SUMMARY AND CONCLUSIONS}

In this work, the spectral properties of the neutral $\rho$ meson are studied at finite temperature and density in a constant external magnetic field using the real time formalism of finite temperature field theory. The effective $\rho \pi \pi$ and $\rho N N$ interactions are considered for the evaluation of the one-loop self-energy of $\rho^{0}$. Accordingly, the magnetically modified in-medium propagators for pions and protons are used and contain an infinite sum over the Landau levels, implying no constraint on the strength of the external magnetic field. From the self-energy, the 
eB-dependent vacuum part is extracted by means of dimensional regularization in which the ultraviolet divergence corresponding to the pure-vacuum self-energy is isolated as the pole of gamma and Hurwitz zeta functions. It is shown that the external magnetic field does not create additional divergences so that the vacuum counterterms required in the absence of the background field remain sufficient to renormalize the theory at nonzero magnetic field.

The general Lorentz structure for the in-medium massive vector boson self-energy in the presence of external magnetic field has been constructed with four linearly independent basis tensors, out of which three form a mutually orthogonal set. Thus, the extraction of the form factors from the self-energy becomes considerably simple. Moreover, it is shown that, with vanishing perpendicular momentum of the external particle, one can arrive at a new set of constraint relations among the form factors. As a result, there remain only two form factors to be determined from the self-energy. As a consistency check, the numerical $B \rightarrow 0$ limit of the real as well as imaginary parts of the form factors is shown to reproduce the zero field results. Solving the Dyson-Schwinger equation with the one-loop self-energy, the complete interacting $\rho^{0}$ propagator is obtained. Consequently, two distinct modes are observed in the study of the effective mass, dispersion relations and the spectral function of $\rho^{0}$ where one of the modes (modeA) possesses twofold degeneracy. It is known [31,38] that nontrivial Landau cuts appear in the presence of external magnetic field along with finite temperature even if the loop particles are of equal mass, which is completely a magnetic field effect. However, in contrast to mode-A, the nontrivial Landau cut is found to be absent in the case of mode-B. Also, a sharper decrease in the effective mass is observed for the latter, which essentially stems from the dominant eB-dependent vacuum contribution in the real part of the corresponding form factor.

Finally, the decay width for the $\rho^{0} \rightarrow \pi^{+} \pi^{-}$channel is obtained for the two distinct modes and is found to become zero at certain critical values of magnetic field depending upon the temperature and baryon chemical potential. The corresponding variation of the critical field with these external parameters shows an increasing trend for large baryonic chemical potential. However, it is observed that both the distinct modes possess a maximum value of $\mu_{B}$ below which the temperature dependence gets reversed. Especially, at a given temperature (say $T=160 \mathrm{MeV}$ ), $e B_{c}$ attains the lowest values $\left(123 \mathrm{MeV}^{2}\right.$ for mode- $\mathrm{A}$ and $116 \mathrm{MeV}^{2}$ for mode-B) in case of zero chemical potential. In Ref. [27], charged $\rho$ meson condensation has been studied at finite temperature and density. For charged $\rho$ mesons, the critical field for which the vector meson mass vanishes is observed to lie in the range of $0.2-0.6 \mathrm{GeV}^{2}$ at zero density with temperature in the range $0.2-0.5 \mathrm{GeV}$. However, in the case of $\rho^{0}$, the absence of the trivial Landau shift in the energy eigenvalue results in a much slower decrease in the effective mass. As a consequence, unrealistically high magnetic field values are required to observe neutral $\rho$ condensation in the presence of temperature and medium (see Fig. 14). In this scenario, the suppression in the $\rho^{0} \rightarrow \pi^{+} \pi^{-}$channel can serve as an important alternative. Magnetic modification of $\rho$ meson properties studied in this work deals with effective hadronic interactions. Thus, the observable modification can only occur if the initial burst of magnetic field survives up to hadronization, retaining an appreciable field strength. However, the recent report $[56,57]$ argued that the observed suppression in the branching ratio of the $\rho^{0} \rightarrow \pi^{+} \pi^{-}$channel in the central collisions $(B \sim 0)$ is due to the rescattering mechanism in the hadronic medium, implying that the magnetic field effects in the neutral $\rho$ decay are negligible in HIC experiments. On the other hand, the present study can be relevant in situations present inside magnetars.

\section{ACKNOWLEDGMENTS}

S. G. acknowledges the Indian Institute of Technology Gandhinagar for the postdoctoral fellowship.

\section{APPENDIX A: USEFUL IDENTITIES}

We have the following list of $d$-dimensional integrals in Minkowski space [60]:

$$
\begin{aligned}
\int \frac{d^{d} k}{(2 \pi)^{d}} \frac{1}{\left(k^{2}-\Delta\right)^{n}}= & \frac{i(-1)^{n}}{(4 \pi)^{d / 2}} \frac{\Gamma(n-d / 2)}{\Gamma(n)}\left(\frac{1}{\Delta}\right)^{n-d / 2} \\
\int \frac{d^{d} k}{(2 \pi)^{d}} \frac{k^{2}}{\left(k^{2}-\Delta\right)^{n}}= & \frac{i(-1)^{n-1}}{(4 \pi)^{d / 2}}\left(\frac{d}{2}\right) \\
& \times \frac{\Gamma(n-1-d / 2)}{\Gamma(n)}\left(\frac{1}{\Delta}\right)^{n-1-d / 2}
\end{aligned}
$$

$$
\begin{aligned}
\int \frac{d^{d} k}{(2 \pi)^{d}} \frac{k^{\mu} k^{\nu}}{\left(k^{2}-\Delta\right)^{n}}= & \frac{i(-1)^{n-1}}{(4 \pi)^{d / 2}}\left(\frac{g^{\mu \nu}}{2}\right) \\
& \times \frac{\Gamma(n-1-d / 2)}{\Gamma(n)}\left(\frac{1}{\Delta}\right)^{n-1-d / 2} .
\end{aligned}
$$

Using the orthogonality properties of the generalized Laguerre polynomials, one can derive the following identities,

$$
\begin{aligned}
& \int \frac{d^{2} k_{\perp}}{(2 \pi)^{2}} e^{-2 \alpha_{k}} L_{l}\left(2 \alpha_{k}\right) L_{n}\left(2 \alpha_{k}\right) k_{\perp}^{\mu} k_{\perp}^{\nu} \\
& =-g_{\perp}^{\mu \nu} \frac{(e B)^{2}}{32 \pi}\left[(2 n+1) \delta_{l}^{n}-(n+1) \delta_{l}^{n+1}-n \delta_{l}^{n-1}\right] \\
& \int \frac{d^{2} k_{\perp}}{(2 \pi)^{2}} e^{-2 \alpha_{k}} L_{l}\left(2 \alpha_{k}\right) L_{n}\left(2 \alpha_{k}\right)=\frac{e B}{8 \pi} \delta_{l}^{n}
\end{aligned}
$$




$$
\begin{aligned}
& \int \frac{d^{2} k_{\perp}}{(2 \pi)^{2}} e^{-2 \alpha_{k}} L_{l-1}^{1}\left(2 \alpha_{k}\right) L_{n-1}^{1}\left(2 \alpha_{k}\right) k_{\perp}^{\mu} k_{\perp}^{\nu}=-g_{\perp}^{\mu \nu} \frac{(e B)^{2}}{32 \pi} n \delta_{l-1}^{n-1} \\
& (\mathrm{~A} 6) \\
& \frac{d^{2} k_{\perp}}{(2 \pi)^{2}} e^{-2 \alpha_{k}} L_{l-1}^{1}\left(2 \alpha_{k}\right) L_{n-1}^{1}\left(2 \alpha_{k}\right) k_{\perp}^{2}=-\frac{(e B)^{2}}{16 \pi} n \delta_{l-1}^{n-1},
\end{aligned}
$$$$
\left(\Pi_{\pi}^{\mu \nu}\right)_{\text {pure-vac }}(q)
$$$$
=i \int \frac{d^{4} k}{(2 \pi)^{4}} \frac{\mathcal{N}_{\pi}^{\mu \nu}(q, k)}{\left(k^{2}-m_{\pi}^{2}+i \epsilon\right)\left((q+k)^{2}-m_{\pi}^{2}+i \epsilon\right)}
$$

where $\alpha_{k}=-k_{\perp}^{2} / e B$.

\section{APPENDIX B: CALCULATION OF VACUUM SELF-ENERGY}

$$
\begin{aligned}
& \left(\Pi_{\mathrm{N}}^{\mu \nu}\right)_{\text {pure-vac }}(q) \\
& \quad=i \int \frac{d^{4} k}{(2 \pi)^{4}} \frac{\mathcal{N}_{\mathrm{N}}^{\mu \nu}(q, k)}{\left(k^{2}-m_{N}^{2}+i \epsilon\right)\left((q+k)^{2}-m_{N}^{2}+i \epsilon\right)},
\end{aligned}
$$

In order to evaluate the momentum integrals in Eqs. (3) and (4), they are rewritten as

where $\mathcal{N}_{\mathrm{N}}^{\mu \nu}(q, k)$ contains the trace over Dirac matrices:

$$
\begin{aligned}
\mathcal{N}_{\mathrm{N}}^{\mu \nu}(q, k)= & -2 g_{\rho N N}^{2} \operatorname{Tr}\left[\Gamma^{\nu}(q)\left(q+\not k+m_{N}\right) \Gamma^{\mu}(-q)\left(\not k+m_{N}\right)\right] \\
= & -8 g_{\rho N N}^{2}\left[\left(m_{N}^{2}-k^{2}-k \cdot q\right) g^{\mu \nu}+2 k^{\mu} k^{\nu}+\left(q^{\mu} k^{\nu}+q^{\nu} k^{\mu}\right)+\kappa_{\rho}\left(q^{2} g^{\mu \nu}-q^{\mu} q^{\nu}\right)\right. \\
& \left.+\frac{\kappa_{\rho}^{2}}{4 m_{N}^{2}}\left\{\left(m_{N}^{2}+k^{2}-k \cdot q\right)\left(q^{2} g^{\mu \nu}-q^{\mu} q^{\nu}\right)-2 q^{2} k^{\mu} k^{\nu}-2(k \cdot q)^{2} g^{\mu \nu}+2(k \cdot q)\left(q^{\mu} k^{\nu}+q^{\nu} k^{\mu}\right)\right\}\right] .
\end{aligned}
$$

Applying standard Feynman paramerization, the denominators of Eqs. (B1) and (B2) are combined to get

$$
\begin{aligned}
& \left(\Pi_{\pi}^{\mu \nu}\right)_{\text {pure-vac }}(q) \\
& =\left.i \int_{0}^{1} d x \int \frac{d^{d} k}{(2 \pi)^{d}} \Lambda_{\pi}^{2-d / 2} \frac{\mathcal{N}_{\pi}^{\mu \nu}(q, k)}{\left[(k+x q)^{2}-\Delta_{\pi}\right]^{2}}\right|_{d \rightarrow 4} \\
& \left(\Pi_{\mathrm{N}}^{\mu \nu}\right)_{\text {pure-vac }}(q) \\
& =\left.i \int_{0}^{1} d x \int \frac{d^{d} k}{(2 \pi)^{d}} \Lambda_{\mathrm{N}}^{2-d / 2} \frac{\mathcal{N}_{\mathrm{N}}^{\mu \nu}(q, k)}{\left[(k+x q)^{2}-\Delta_{\mathrm{N}}\right]^{2}}\right|_{d \rightarrow 4},
\end{aligned}
$$

where

$$
\begin{aligned}
& \Delta_{\pi}=m_{\pi}^{2}-x(1-x) q^{2}-i \epsilon \\
& \Delta_{\mathrm{N}}=m_{N}^{2}-x(1-x) q^{2}-i \epsilon
\end{aligned}
$$

and the space-time dimension has been changed from 4 to $d$ in order to work with the dimensional regularization so that the additional scale parameters $\Lambda_{\pi}$ and $\Lambda_{\mathrm{N}}$ of dimension $\mathrm{GeV}^{2}$ have been introduced to keep the overall dimension of the self-energy the same. It is now straightforward to perform the momentum integrals of the above equations after a momentum shift $k \rightarrow(k-x q)$ using the identities provided in Appendix A, so that the vacuum self-energies become

$$
\begin{aligned}
& \left(\Pi_{\pi}^{\mu \nu}\right)_{\text {pure-vac }}(q) \\
& =\left.\left(q^{2} g^{\mu \nu}-q^{\mu} q^{\nu}\right)\left(\frac{g_{\rho \pi \pi}^{2} q^{2}}{32 \pi^{2}}\right) \int_{0}^{1} d x \Gamma(\varepsilon-1)\left(\frac{\Delta_{\pi}}{4 \pi \Lambda_{\pi}}\right)^{-\varepsilon}\right|_{\varepsilon \rightarrow 0} \\
& \left(\Pi_{\mathrm{N}}^{\mu \nu}\right)_{\text {pure-vac }}(q) \\
& =\left(q^{2} g^{\mu \nu}-q^{\mu} q^{\nu}\right)\left(\frac{g_{\rho N N}^{2}}{2 \pi^{2}}\right) \int_{0}^{1} d x\left[\left\{2 x(1-x)+\kappa_{\rho}+\frac{\kappa_{\rho}^{2}}{2}\right\} \Gamma(\varepsilon)\right. \\
& \left.+\frac{\kappa_{\rho}^{2}}{4 m_{N}^{2}} \Delta_{\mathrm{N}} \Gamma(\varepsilon-1)\right]\left.\left(\frac{\Delta_{\mathrm{N}}}{4 \pi \Lambda_{\mathrm{N}}}\right)^{-\varepsilon}\right|_{\varepsilon \rightarrow 0},
\end{aligned}
$$

where $\varepsilon=(2-d / 2)$. Expanding the above equations about $\varepsilon=0$, we get

$$
\begin{aligned}
\left(\Pi_{\pi}^{\mu \nu}\right)_{\text {pure-vac }}(q)= & \left(q^{2} g^{\mu \nu}-q^{\mu} q^{\nu}\right)\left(\frac{-g_{\rho \pi \pi}^{2} q^{2}}{32 \pi^{2}}\right) \int_{0}^{1} d x \Delta_{\pi} \\
& \times\left.\left[\frac{1}{\varepsilon}-\gamma_{\mathrm{E}}+1-\ln \left(\frac{\Delta_{\pi}}{4 \pi \Lambda_{\pi}}\right)\right]\right|_{\varepsilon \rightarrow 0} \quad(\mathrm{~B} 10) \\
\left(\Pi_{\mathrm{N}}^{\mu \nu}\right)_{\text {pure-vac }}(q)= & \left(q^{2} g^{\mu \nu}-q^{\mu} q^{\nu}\right)\left(\frac{g_{\rho N N}^{2}}{2 \pi^{2}}\right) \\
& \times \int_{0}^{1} d x\left[\left\{2 x(1-x)+\kappa_{\rho}+\frac{\kappa_{\rho}^{2}}{2}-\frac{\kappa_{\rho}^{2}}{4 m_{N}^{2}} \Delta_{\mathrm{N}}\right\}\right. \\
& \left.\times\left\{\frac{1}{\varepsilon}-\gamma_{\mathrm{E}}-\ln \left(\frac{\Delta_{\mathrm{N}}}{4 \pi \Lambda_{\mathrm{N}}}\right)\right\}-\frac{\kappa_{\rho}^{2}}{4 m_{N}^{2}} \Delta_{\mathrm{N}}\right]\left.\right|_{\varepsilon \rightarrow 0},
\end{aligned}
$$

where $\gamma_{\mathrm{E}}$ is the Euler-Mascheroni constant. 


\section{APPENDIX C: CALCULATION OF EB-DEPENDENT VACUUM CONTRIBUTION FOR $\pi \pi$ LOOP}

In this Appendix, we sketch how to obtain Eqs. (46) and (48). We rewrite Eq. (44) as

$$
\begin{aligned}
&\left(\Pi_{\pi}^{\mu \nu}\right)_{\mathrm{vac}}(q, e B) \\
&= i \sum_{l=0}^{\infty} \sum_{n=0}^{\infty} \int \frac{d^{2} k_{\|}}{(2 \pi)^{2}} \\
& \quad \times \int \frac{d^{2} k_{\perp}}{(2 \pi)^{2}} \frac{\mathcal{N}_{\pi, n l}^{\mu \nu}(q, k)}{\left(k_{\|}^{2}-m_{l}^{2}+i \epsilon\right)\left(\left(q_{\|}+k_{\|}\right)^{2}-m_{n}^{2}+i \epsilon\right)} .
\end{aligned}
$$

For the simplicity in analytic calculations, we take the transverse momentum of the $\rho^{0}$ to be zero, i.e., $q_{\perp}=0$. This implies that the $d^{2} k_{\perp}$ integration can be performed analytically using the orthogonality of the Laguerre polynomial, the details of which can be obtained from Appendix E, so that the self-energy becomes

$$
\begin{aligned}
& \left(\Pi_{\pi}^{\mu \nu}\right)_{\mathrm{vac}}\left(q_{\|}, e B\right) \\
& =i \sum_{l=0}^{\infty} \sum_{n=0}^{\infty} \int \frac{d^{2} k_{\|}}{(2 \pi)^{2}} \frac{\tilde{\mathcal{N}}_{\pi, n l}^{\mu \nu}\left(q_{\|}, k_{\|}\right)}{\left(k_{\|}^{2}-m_{l}^{2}+i \epsilon\right)\left(\left(q_{\|}+k_{\|}\right)^{2}-m_{n}^{2}+i \epsilon\right)},
\end{aligned}
$$

where $\tilde{\mathcal{N}}_{\pi, n l}^{\mu \nu}\left(q_{\|}, k_{\|}\right)$is given in Eq. (E5). Next, we use the standard Feynman parametrization technique to combine the denominators of Eq. (C2) and change the reduced space-time dimension from 2 to $d$ in order to apply the dimensional regularization for which a scale parameter $\Lambda_{\pi}$ of dimension $\mathrm{GeV}^{2}$ has to be introduced in order to keep the overall dimension of the self-energy the same. This leads to

$$
\begin{aligned}
& \left(\Pi_{\pi}^{\mu \nu}\right)_{\mathrm{vac}}\left(q_{\|}, e B\right) \\
& =\left.i \sum_{l=0}^{\infty} \sum_{n=0}^{\infty} \int_{0}^{1} d x \int \frac{d^{d} k_{\|}}{(2 \pi)^{d}} \Lambda_{\pi}^{1-d / 2} \frac{\tilde{\mathcal{N}}_{\pi, n l}^{\mu \nu}\left(q_{\|}, k_{\|}\right)}{\left[\left(k_{\|}+x q_{\|}\right)^{2}-\Delta_{n l}^{\pi}\right]^{2}}\right|_{d \rightarrow 2},
\end{aligned}
$$

where

$$
\Delta_{n l}^{\pi}=\Delta_{\pi}\left(q_{\perp}=0\right)+2 e B\{l+1-x(l-n)\}
$$

with $\Delta_{\pi}$ defined in Eq. (B6). It is now trivial to perform the $d^{d} k_{\|}$integration after a shift of momentum $k_{\|} \rightarrow\left(k_{\|}-x q_{\|}\right)$ using the identities provided in Appendix A, so that the self-energy becomes

$$
\begin{aligned}
\left(\Pi_{\pi}^{\mu \nu}\right)_{\mathrm{vac}}\left(q_{\|}, e B\right) & \\
= & \frac{-g_{\rho \pi \pi}^{2} q_{\|}^{2}}{16 \pi^{2}} e B \int_{0}^{1} d x \sum_{n=0}^{\infty} \sum_{l=(n-1)}^{(n+1)}(-1)^{n+l}\left(4 \pi \Lambda_{\pi}\right)^{\varepsilon} \\
& \times\left[-\left(q_{\|}^{2} g_{\|}^{\mu \nu}-q_{\|}^{\mu} q_{\|}^{\nu}\right) \delta_{l}^{n} \Gamma(\varepsilon)\left(\Delta_{n l}^{\pi}\right)^{-\varepsilon}\right. \\
& -q_{\|}^{2} g_{\perp}^{\mu \nu} \frac{e B}{2}\left\{(2 n+1) \delta_{l}^{n}-(n+1) \delta_{l}^{n+1}-n \delta_{l}^{n-1}\right\} \\
& \left.\times \Gamma(\varepsilon+1)\left(\Delta_{n l}^{\pi}\right)^{-\varepsilon-1}\right]\left.\right|_{\varepsilon \rightarrow 0},
\end{aligned}
$$

where $\varepsilon=(1-d / 2)$ and the presence of Kronecker delta functions in Eq. (E5) has made the double sum into a single one or, in other words, the sum over index $l$ runs only from $(n-1)$ to $(n+1)$. The infinite sum in the above equations can be expressed in terms of the Hurwitz zeta function so that we get, after some simplifications,

$$
\begin{aligned}
\left(\Pi_{\pi}^{\mu \nu}\right)_{\mathrm{vac}}\left(q_{\|}, e B\right)= & \frac{-g_{\rho \pi \pi}^{2} q_{\|}^{2}}{16 \pi^{2}} e B \int_{0}^{1} d x\left(\frac{4 \pi \Lambda_{\pi}}{2 e B}\right)^{\varepsilon}\left[-\left(q_{\|}^{2} g_{\|}^{\mu \nu}-q_{\|}^{\mu} q_{\|}^{\nu}\right) \Gamma(\varepsilon) \zeta\left(\varepsilon, z_{\pi}+\frac{1}{2}\right)-\frac{q_{\|}^{2}}{2} g_{\perp}^{\mu \nu} \Gamma(\varepsilon+1)\right. \\
& \left.\times\left\{\zeta\left(\varepsilon, z_{\pi}+\frac{1}{2}\right)+\zeta\left(\varepsilon, z_{\pi}+x+\frac{1}{2}\right)-z_{\pi} \zeta\left(\varepsilon+1, z_{\pi}+\frac{1}{2}\right)-z_{\pi} \zeta\left(\varepsilon+1, z_{\pi}+x+\frac{1}{2}\right)\right\}\right]\left.\right|_{\varepsilon \rightarrow 0},
\end{aligned}
$$

where $z_{\pi}=\frac{\Delta_{\pi}\left(q_{\perp}=0\right)}{2 e B}$. Expanding the above equation about $\varepsilon=0$, we get

$$
\begin{aligned}
\left(\Pi_{\pi}^{\mu \nu}\right)_{\mathrm{vac}}\left(q_{\|}, e B\right)= & \frac{-g_{\rho \pi \pi}^{2} q_{\|}^{2}}{32 \pi^{2}} \int_{0}^{1} d x\left[\left\{\frac{1}{\varepsilon}-\gamma_{\mathrm{E}}+\ln \left(\frac{4 \pi \Lambda_{\pi}}{2 e B}\right)\right\} \Delta_{\pi}\left(q_{\perp}=0\right)\left(q_{\|}^{2} g^{\mu \nu}-q_{\|}^{\mu} q_{\|}^{\nu}\right)\right. \\
& -\left(q_{\|}^{2} g_{\|}^{\mu \nu}-q_{\|}^{\mu} q_{\|}^{\nu}\right) 2 e B\left\{\ln \Gamma\left(z_{\pi}+\frac{1}{2}\right)-\ln \sqrt{2 \pi}\right\} \\
& \left.+q_{\|}^{2} g_{\perp}^{\mu \nu}\left\{\Delta_{\pi}\left(q_{\perp}=0\right)+\frac{e B}{2}-\frac{1}{2} \Delta_{\pi}\left(q_{\perp}=0\right)\left\{\psi\left(z_{\pi}+\frac{1}{2}\right)+\psi\left(z_{\pi}+x+\frac{1}{2}\right)\right\}\right\}\right]\left.\right|_{\varepsilon \rightarrow 0},
\end{aligned}
$$


where $\psi(z)$ is the digamma function. It is now trivial to check that, in the limit $e B \rightarrow 0$, the above equation exactly boils down to the pure-vacuum contribution given in Eq. (9). Thus, extracting the pure-vacuum contribution from the above equation, we get

$$
\left(\Pi_{\pi}^{\mu \nu}\right)_{\mathrm{vac}}\left(q_{\|}, e B\right)=\left(\Pi_{\pi}^{\mu \nu}\right)_{\text {pure-vac }}\left(q_{\|}\right)+\left(\Pi_{\pi}^{\mu \nu}\right)_{e B \text {-vac }}\left(q_{\|}, e B\right)
$$

where

$$
\begin{aligned}
\left(\Pi_{\pi}^{\mu \nu}\right)_{e B \text {-vac }}\left(q_{\|}, e B\right)= & \frac{-g_{\rho \pi \pi}^{2} q_{\|}^{2}}{32 \pi^{2}} \int_{0}^{1} d x\left[\left\{\ln \left(\frac{\Delta_{\pi}\left(q_{\perp}=0\right)}{2 e B}\right)-1\right\} \Delta_{\pi}\left(q_{\perp}=0\right)\left(q_{\|}^{2} g^{\mu \nu}-q_{\|}^{\mu} q_{\|}^{\nu}\right)\right. \\
& -\left(q_{\|}^{2} g_{\|}^{\mu \nu}-q_{\|}^{\mu} q_{\|}^{\nu}\right) 2 e B\left\{\ln \Gamma\left(z_{\pi}+\frac{1}{2}\right)-\ln \sqrt{2 \pi}\right\} \\
& \left.+q_{\|}^{2} g_{\perp}^{\mu \nu}\left\{\Delta_{\pi}\left(q_{\perp}=0\right)+\frac{e B}{2}-\frac{1}{2} \Delta_{\pi}\left(q_{\perp}=0\right)\left\{\psi\left(z_{\pi}+\frac{1}{2}\right)+\psi\left(z_{\pi}+x+\frac{1}{2}\right)\right\}\right\}\right],
\end{aligned}
$$

which is finite and independent of scale.

\section{APPENDIX D: CALCULATION OF eB-DEPENDENT VACUUM CONTRIBUTION FOR PROTON-PROTON LOOP}

In this Appendix, we sketch how to obtain Eqs. (47) and (49) We rewrite Eq. (45) as

$$
\begin{aligned}
\left(\Pi_{\mathrm{p}}^{\mu \nu}\right)_{\mathrm{vac}}(q, e B)= & i \sum_{l=0}^{\infty} \sum_{n=0}^{\infty} \int \frac{d^{2} k_{\|}}{(2 \pi)^{2}} \int \frac{d^{2} k_{\perp}}{(2 \pi)^{2}} \\
& \times \frac{\mathcal{N}_{\mathrm{p}, n l}^{\mu \nu}(q, k)}{\left(k_{\|}^{2}-M_{l}^{2}+i \epsilon\right)\left(\left(q_{\|}+k_{\|}\right)^{2}-M_{n}^{2}+i \epsilon\right)},
\end{aligned}
$$

where $\mathcal{N}_{\mathrm{p}, n l}^{\mu \nu}(q, k)$ is given in Eq. (42). For simplicity in analytic calculations, we take the transverse momentum of the $\rho^{0}$ to be zero, i.e., $q_{\perp}=0$. This implies that the $d^{2} k_{\perp}$ integration can be performed analytically using the orthogonality of the Laguerre polynomial, the details of which can be obtained from Appendix E, so that the self-energy becomes

$$
\begin{aligned}
& \left(\Pi_{\mathrm{p}}^{\mu \nu}\right)_{\mathrm{vac}}\left(q_{\|}, e B\right) \\
& =i \sum_{l=0}^{\infty} \sum_{n=0}^{\infty} \int \frac{d^{2} k_{\|}}{(2 \pi)^{2}} \frac{\tilde{\mathcal{N}}_{\mathrm{p}, n l}^{\mu \nu}\left(q_{\|}, k_{\|}\right)}{\left(k_{\|}^{2}-M_{l}^{2}+i \epsilon\right)\left(\left(q_{\|}+k_{\|}\right)^{2}-M_{n}^{2}+i \epsilon\right)},
\end{aligned}
$$

where $\tilde{\mathcal{N}}_{\mathrm{p}, n l}^{\mu \nu}\left(q_{\|}, k_{\|}\right)$can be read off from Eq. (E8). Next, we use the standard Feynman parametrization technique to combine the denominators of Eq. (D2) and change the reduced space-time dimension from 2 to $d$ in order to apply the dimensional regularization for which a scale parameter $\Lambda_{N}$ of dimension $\mathrm{GeV}^{2}$ has to be introduced in order to keep the overall dimension of the self-energy the same. This leads to

$$
\begin{aligned}
\left(\Pi_{\mathrm{p}}^{\mu \nu}\right)_{\mathrm{vac}}\left(q_{\|}, e B\right)= & i \sum_{l=0}^{\infty} \sum_{n=0}^{\infty} \int_{0}^{1} d x \int \frac{d^{d} k_{\|}}{(2 \pi)^{d}} \Lambda_{N}^{1-d / 2} \\
& \times\left.\frac{\tilde{\mathcal{N}}_{\mathrm{p}, n l}^{\mu \nu}\left(q_{\|}, k_{\|}\right)}{\left[\left(k_{\|}+x q_{\|}\right)^{2}-\Delta_{n l}^{\mathrm{p}}\right]^{2}}\right|_{d \rightarrow 2},
\end{aligned}
$$

where

$$
\Delta_{n l}^{\mathrm{p}}=\Delta_{N}\left(q_{\perp}=0\right)+2 e B\{l-x(l-n)\}
$$

with $\Delta_{N}$ defined in Eq. (B7). It is now trivial to perform the $d^{d} k_{\|}$integration after a shift of momentum $k_{\|} \rightarrow\left(k_{\|}-\right.$ $\left.x q_{\|}\right)$using the identities provided in Appendix A, so that the self-energy becomes 


$$
\begin{aligned}
\left(\Pi_{\mathrm{p}}^{\mu \nu}\right)_{\mathrm{vac}}\left(q_{\|}, e B\right)= & \frac{g_{\rho N N}^{2}}{4 \pi^{2}} e B \int_{0}^{1} d x \sum_{n=0}^{\infty} \sum_{l=(n-1)}^{(n+1)}(-1)^{n+l}\left(4 \pi \Lambda_{\pi}\right)^{\varepsilon}\left[\left[4 e B g_{\|}^{\mu \nu} n \delta_{l-1}^{n-1}\right.\right. \\
& \left.+\left\{\left(m_{N}^{2}+x(1-x) q_{\|}^{2}\right) g_{\|}^{\mu \nu}-2 x(1-x) q_{\|}^{\mu} q_{\|}^{\nu}\right\}\left(\delta_{l-1}^{n-1}+\delta_{l}^{n}\right)-\left(m_{N}^{2}+x(1-x) q_{\|}^{2}\right) g_{\perp}^{\mu \nu}\left(\delta_{l-1}^{n}+\delta_{l}^{n-1}\right)\right] \\
& \times \Gamma(\varepsilon+1)\left(\Delta_{n l}^{\mathrm{p}}\right)^{-\varepsilon-1}-\left\{g_{\|}^{\mu \nu}\left(\delta_{l-1}^{n-1}+\delta_{l}^{n}\right) \varepsilon+g_{\perp}^{\mu \nu}\left(\delta_{l-1}^{n}+\delta_{l}^{n-1}\right)(-\varepsilon+1)\right\} \Gamma(\varepsilon)\left(\Delta_{n l}^{\mathrm{p}}\right)^{-\varepsilon} \\
& +\kappa_{\rho}\left\{\left(q_{\|}^{2} g_{\|}^{\mu \nu}-q_{\|}^{\mu} q_{\|}^{\nu}\right)\left(\delta_{l-1}^{n-1}+\delta_{l}^{n}\right)-q_{\|}^{2} g_{\perp}^{\mu \nu}\left(\delta_{l-1}^{n}+\delta_{l}^{n-1}\right)\right\} \Gamma(\varepsilon+1)\left(\Delta_{n l}^{\mathrm{p}}\right)^{-\varepsilon-1} \\
& +\frac{\kappa_{\rho}^{2}}{4 m_{N}^{2}}\left[\left\{-4 e B n \delta_{l-1}^{n-1}+\left(m_{N}^{2}+x(1-x) q_{\|}^{2}\right)\left(\delta_{l-1}^{n-1}+\delta_{l}^{n}\right)\right\}\left(q_{\|}^{2} g_{\|}^{\mu \nu}-q_{\|}^{\mu} q_{\|}^{\nu}\right)\right. \\
& \left.-q_{\|}^{2}\left(m_{N}^{2}+x(1+x) q_{\|}^{2}\right) g_{\perp}^{\mu \nu}\left(\delta_{l-1}^{n}+\delta_{l}^{n-1}\right)\right] \Gamma(\varepsilon+1)\left(\Delta_{n l}^{\mathrm{p}}\right)^{-\varepsilon-1} \\
& \left.-\frac{\kappa_{\rho}^{2}}{4 m_{N}^{2}}\left\{\left(q_{\|}^{2} g_{\|}^{\mu \nu}-q_{\|}^{\mu} q_{\|}^{\nu}\right)(-\varepsilon-1)\left(\delta_{l-1}^{n-1}+\delta_{l}^{n}\right)+q_{\|}^{2} g_{\perp}^{\mu \nu}\left(\delta_{l-1}^{n}+\delta_{l}^{n-1}\right) \varepsilon\right\} \Gamma(\varepsilon)\left(\Delta_{n l}^{\mathrm{p}}\right)^{-\varepsilon}\right]\left.\right|_{\varepsilon \rightarrow 0}
\end{aligned}
$$

where $\varepsilon=(1-d / 2)$ and the presence of Kronecker delta functions in Eq. (E8) has made the double sum into a single one or, in other words, the sum over index $l$ runs only from $(n-1)$ to $(n+1)$. The infinite sum in the above equations can be expressed in terms of the Hurwitz zeta function so that we get, after some simplifications,

$$
\begin{aligned}
\left(\Pi_{\mathrm{p}}^{\mu \nu}\right)_{\mathrm{vac}}\left(q_{\|}, e B\right)= & \frac{g_{\rho \pi \pi}^{2}}{4 \pi^{2}} \int_{0}^{1} d x\left(\frac{4 \pi \Lambda_{N}}{2 e B}\right)^{\varepsilon}\left[\left[2 e B g_{\|}^{\mu \nu}\left\{\zeta\left(\varepsilon, z_{N}\right)-z_{N} \zeta\left(\varepsilon+1, z_{N}\right)\right\}\right.\right. \\
& +\left\{\left(m_{N}^{2}+x(1-x) q_{\|}^{2}\right) g_{\|}^{\mu \nu}-2 x(1-x) q_{\|}^{\mu} q_{\|}^{\nu}\right\}\left\{\zeta\left(\varepsilon+1, z_{N}\right)-\frac{1}{2} z_{N}^{-\varepsilon-1}\right\} \\
& \left.+\left(m_{N}^{2}+x(1-x) q_{\|}^{2}\right) g_{\perp}^{\mu \nu} \zeta\left(\varepsilon+1, z_{N}+x\right)\right] \Gamma(\varepsilon+1)-2 e B\left\{g_{\|}^{\mu \nu} \varepsilon\left(\zeta\left(\varepsilon, z_{N}\right)-\frac{1}{2} z_{N}^{-\varepsilon}\right)\right. \\
& \left.+g_{\perp}^{\mu \nu}(\varepsilon-1) \zeta\left(\varepsilon, z_{N}+x\right)\right\} \Gamma(\varepsilon)+\kappa_{\rho}\left\{\left(q_{\|}^{2} g_{\|}^{\mu \nu}-q_{\|}^{\mu} q_{\|}^{\nu}\right)\left(\zeta\left(1+\varepsilon, z_{N}\right)-\frac{1}{2} z_{N}^{-\varepsilon-1}\right)\right. \\
& \left.+q_{\|}^{2} g_{\perp}^{\mu \nu} \zeta\left(\varepsilon+1, z_{N}+x\right)\right\} \Gamma(\varepsilon+1)+\frac{\kappa_{\rho}^{2}}{4 m_{N}^{2}}\left[\left\{-2 e B\left(\zeta\left(\varepsilon, z_{N}\right)-z_{N} \zeta\left(\varepsilon+1, z_{N}\right)\right)\right.\right. \\
& \left.+\left(m_{N}^{2}+x(1-x) q_{\|}^{2}\right)\left(\zeta\left(\varepsilon+1, z_{N}\right)-\frac{1}{2} z_{N}^{-\varepsilon-1}\right)\right\}\left(q_{\|}^{2} g_{\|}^{\mu \nu}-q_{\|}^{\mu} q_{\|}^{\nu}\right) \\
& \left.+q_{\|}^{2} g_{\perp}^{\mu \nu}\left(m_{N}^{2}+x(1-x) q_{\|}^{2}\right) \zeta\left(\varepsilon+1, z_{N}+x\right)\right] \Gamma(\varepsilon+1)+\frac{\kappa_{\rho}^{2}}{4 m_{N}^{2}} 2 e B\left\{\left(q_{\|}^{2} g_{\|}^{\mu \nu}-q_{\|}^{\mu} q_{\|}^{\nu}\right)(\varepsilon+1)\right. \\
& \left.\left.\times\left(\zeta\left(\varepsilon, z_{N}\right)-\frac{1}{2} z_{N}^{-\varepsilon}\right)+q_{\|}^{2} g_{\perp}^{\mu \nu} \varepsilon \zeta\left(\varepsilon, z_{N}+x\right)\right\} \Gamma(\varepsilon)\right]\left.\right|_{\varepsilon \rightarrow 0},
\end{aligned}
$$

where $z_{N}=\frac{\Delta_{N}\left(q_{\perp}=0\right)}{2 e B}$. Expanding the above equation about $\varepsilon=0$, we get

$$
\begin{aligned}
\left(\Pi_{\mathrm{p}}^{\mu \nu}\right)_{\mathrm{vac}}\left(q_{\|}, e B\right)= & \frac{g_{\rho N N}^{2}}{4 \pi^{2}} \int_{0}^{1} d x\left[\left\{\frac{1}{\varepsilon}-\gamma_{\mathrm{E}}+\ln \left(\frac{4 \pi \Lambda_{N}}{2 e B}\right)\right\}\left\{2 x(1-x)+\kappa_{\rho}+\frac{\kappa_{\rho}^{2}}{2}-\frac{\kappa_{\rho}^{2}}{4 m_{N}^{2}} \Delta_{N}\left(q_{\perp}=0\right)\right\}\left(q_{\|}^{2} g^{\mu \nu}-q_{\|}^{\mu} q_{\|}^{\nu}\right)\right. \\
& -2 x(1-x)\left(\psi\left(z_{N}\right)+\frac{1}{2 z_{N}}\right)\left(q_{\|}^{2} g_{\|}^{\mu \nu}-q_{\|}^{\mu} q_{\|}^{\nu}\right)+2 e B g_{\perp}^{\mu \nu}\left\{\left(z_{N}-\frac{m_{N}^{2}}{e B}\right) \psi\left(z_{N}+x\right)+z_{N}\right. \\
& +\ln \Gamma(z+x)-\ln \sqrt{2 \pi}\}-\kappa_{\rho}\left\{\left(q_{\|}^{2} g_{\|}^{\mu \nu}-q_{\|}^{\mu} q_{\|}^{\nu}\right)\left(\psi\left(z_{N}\right)+\frac{1}{2 z_{N}}\right)+q_{\|}^{2} g_{\perp}^{\mu \nu} \psi(z+x)\right\} \\
& +\frac{\kappa_{\rho}^{2}}{4 m_{N}^{2}} 2 e B\left[\left(q_{\|}^{2} g_{\|}^{\mu \nu}-q_{\|}^{\mu} q_{\|}^{\nu}\right)\left\{-\frac{m_{N}^{2}}{e B}\left(\psi\left(z_{N}\right)+\frac{1}{2 z_{N}}\right)+\frac{1}{2} \ln \left(z_{N}\right)+\ln \Gamma\left(z_{N}\right)-\ln \sqrt{2 \pi}\right\}\right. \\
& \left.-q_{\|}^{2} g_{\perp}^{\mu \nu}\left\{\left(\frac{m_{N}^{2}}{e B}-z_{N}\right) \psi\left(z_{N}+x\right)+\Delta_{N}\left(q_{\perp}=0\right)\right\}\right]\left.\right|_{\varepsilon \rightarrow 0}
\end{aligned}
$$


It is now trivial to check that, in the limit $e B \rightarrow 0$, the above equation exactly boils down to the $\frac{1}{2}$ times pure-vacuum contribution given in Eq. (10). Thus, extracting the pure-vacuum contribution from the above equation, we get

$$
\left(\Pi_{\mathrm{p}}^{\mu \nu}\right)_{\mathrm{vac}}\left(q_{\|}, e B\right)=\frac{1}{2}\left(\Pi_{\mathrm{N}}^{\mu \nu}\right)_{\text {pure-vac }}\left(q_{\|}\right)+\left(\Pi_{\mathrm{p}}^{\mu \nu}\right)_{e B \text {-vac }}\left(q_{\|}, e B\right),
$$

where

$$
\begin{aligned}
\left(\Pi_{\mathrm{p}}^{\mu \nu}\right)_{e B-\mathrm{vac}}\left(q_{\|}, e B\right)= & \frac{g_{\rho N N}^{2}}{4 \pi^{2}} \int_{0}^{1} d x\left[\ln \left(\frac{\Delta_{N}\left(q_{\perp}=0\right)}{2 e B}\right)\left\{2 x(1-x)+\kappa_{\rho}+\frac{\kappa_{\rho}^{2}}{2}-\frac{\kappa_{\rho}^{2}}{4 m_{N}^{2}} \Delta_{N}\left(q_{\perp}=0\right)\right\}\left(q_{\|}^{2} g^{\mu \nu}-q_{\|}^{\mu} q_{\|}^{\nu}\right)\right. \\
& -2 x(1-x)\left(\psi\left(z_{N}\right)+\frac{1}{2 z_{N}}\right)\left(q_{\|}^{2} g_{\|}^{\mu \nu}-q_{\|}^{\mu} q_{\|}^{\nu}\right)+2 e B g_{\perp}^{\mu \nu}\left\{\left(z_{N}-\frac{m_{N}^{2}}{e B}\right) \psi\left(z_{N}+x\right)+z_{N}\right. \\
& +\ln \Gamma(z+x)-\ln \sqrt{2 \pi}\}-\kappa_{\rho}\left\{\left(q_{\|}^{2} g_{\|}^{\mu \nu}-q_{\|}^{\mu} q_{\|}^{\nu}\right)\left(\psi\left(z_{N}\right)+\frac{1}{2 z_{N}}\right)+q_{\|}^{2} g_{\perp}^{\mu \nu} \psi(z+x)\right\} \\
& +\frac{\kappa_{\rho}^{2}}{4 m_{N}^{2}} 2 e B\left[\left(q_{\|}^{2} g_{\|}^{\mu \nu}-q_{\|}^{\mu} q_{\|}^{\nu}\right)\left\{-\frac{m_{N}^{2}}{e B}\left(\psi\left(z_{N}\right)+\frac{1}{2 z_{N}}\right)+\frac{1}{2} \ln \left(z_{N}\right)+\ln \Gamma\left(z_{N}\right)-\ln \sqrt{2 \pi}\right\}\right. \\
& \left.-q_{\|}^{2} g_{\perp}^{\mu \nu}\left\{\left(\frac{m_{N}^{2}}{e B}-z_{N}\right) \psi\left(z_{N}+x\right)+\Delta_{N}\left(q_{\perp}=0\right)\right\}+\frac{\kappa_{\rho}^{2}}{4 m_{N}^{2}}\left(q_{\|}^{2} g^{\mu \nu}-q_{\|}^{\mu} q_{\|}^{\nu}\right) \Delta_{N}\left(q_{\perp}=0\right)\right],
\end{aligned}
$$

which is finite and independent of scale.

\section{APPENDIX E: ANALYTIC EVALUATION OF $d^{2} k_{\perp}$ INTEGRAL FOR $q_{\perp}=0$}

In this Appendix, we will calculate the quantities

$$
\begin{aligned}
& \tilde{\mathcal{N}}_{\pi, n l}^{\mu \nu}\left(q_{\|}, k_{\|}\right)=\int \frac{d^{2} k_{\perp}}{(2 \pi)^{2}} \mathcal{N}_{\pi, n l}^{\mu \nu}\left(q_{\|}, q_{\perp}=0, k\right) \\
& \tilde{\mathcal{N}}_{\mathrm{p}, n l}^{\mu \nu}\left(q_{\|}, k_{\|}\right)=\int \frac{d^{2} k_{\perp}}{(2 \pi)^{2}} \mathcal{N}_{\mathrm{p}, n l}^{\mu \nu}\left(q_{\|}, q_{\perp}=0, k\right) .
\end{aligned}
$$

We have the expression for $\mathcal{N}_{\pi, n l}^{\mu \nu}(q, k)$ from Eqs. (40) and (7) as

$$
\begin{aligned}
\mathcal{N}_{\pi, n l}^{\mu \nu}(q, k)= & 4 g_{\rho \pi \pi}^{2}(-1)^{n+l} e^{-\alpha_{k}-\alpha_{p}} L_{l}\left(2 \alpha_{k}\right) L_{n}\left(2 \alpha_{p}\right) \\
& \times\left[q^{4} k^{\mu} k^{\nu}+(q \cdot k)^{2} q^{\mu} q^{\nu}\right. \\
& \left.-q^{2}(q \cdot k)\left(q^{\mu} k^{\nu}+q^{\nu} k^{\mu}\right)\right]
\end{aligned}
$$

which for $q_{\perp}=0$ becomes

$$
\begin{aligned}
\mathcal{N}_{\pi, n l}^{\mu \nu}\left(q_{\|}, k\right)= & 4 g_{\rho \pi \pi}^{2}(-1)^{n+l} e^{-2 \alpha_{k}} L_{l}\left(2 \alpha_{k}\right) L_{n}\left(2 \alpha_{k}\right) \\
& \times\left[q_{\|}^{4} k^{\mu} k^{\nu}+\left(q_{\|} \cdot k_{\|}\right)^{2} q_{\|}^{\mu} q_{\|}^{\nu}\right. \\
& \left.-q_{\|}^{2}\left(q_{\|} \cdot k_{\|}\right)\left(q_{\|}^{\mu} k^{\nu}+q_{\|}^{\nu} k^{\mu}\right)\right] .
\end{aligned}
$$

We now perform the $d^{2} k_{\perp}$ integration using the orthogonality of the Laguerre polynomial (identities provided in Appendix A) to obtain

$$
\begin{aligned}
\tilde{\mathcal{N}}_{\pi, n l}^{\mu \nu}\left(q_{\|}, k_{\|}\right)= & 4 g_{\rho \pi \pi}^{2}(-1)^{n+l} \frac{e B}{8 \pi}\left[\left\{q_{\|}^{4} k_{\|}^{\mu} k_{\|}^{\nu}+\left(q_{\|} \cdot k_{\|}\right)^{2} q_{\|}^{\mu} q_{\|}^{\nu}-q_{\|}^{2}\left(q_{\|} \cdot k_{\|}\right)\left(q_{\|}^{\mu} k_{\|}^{\nu}+q_{\|}^{\nu} k_{\|}^{\mu}\right)\right\} \delta_{l}^{n}\right. \\
& \left.-q_{\|}^{4} g_{\perp}^{\mu \nu} \frac{e B}{4}\left\{(2 n+1) \delta_{l}^{n}-(n+1) \delta_{l}^{n+1}-n \delta_{l}^{n-1}\right\}\right] .
\end{aligned}
$$

Similarly, $\mathcal{N}_{\mathrm{p}, n l}^{\mu \nu}(q, k)$ is obtained from Eq. (41) as

$$
\mathcal{N}_{\mathrm{p}, n l}^{\mu \nu}(q, k)=-g_{\rho N N}^{2}(-1)^{n+l} e^{-\alpha_{k}-\alpha_{p}} \operatorname{Tr}\left[\Gamma^{\nu}(q) \mathcal{D}_{n}(q+k) \Gamma^{\mu}(-q) \mathcal{D}_{l}(k)\right]
$$

Evaluating the trace over the Dirac matrices in the above equation, we get for $q_{\perp}=0$ (considering the Lorentz symmetric part since the self-energy should be symmetric in the two Lorentz indices) 


$$
\begin{aligned}
\mathcal{N}_{\mathrm{p}, n l}^{\mu \nu}\left(q_{\|}, k\right)= & -8 g_{\rho N N}^{2}(-1)^{n+l} e^{-2 \alpha_{k}}\left[8\left(2 k_{\perp}^{\mu} k_{\perp}^{\nu}-k_{\perp}^{2} g^{\mu \nu}\right) L_{l-1}^{1}\left(2 \alpha_{k}\right) L_{n-1}^{1}\left(2 \alpha_{k}\right)\right. \\
& +\left\{\left(m_{N}^{2}-k_{\|}^{2}-k_{\|} \cdot q_{\|}\right) g_{\|}^{\mu \nu}+2 k_{\|}^{\mu} k_{\|}^{\nu}+\left(q_{\|}^{\mu} k_{\|}^{\nu}+q_{\|}^{\nu} k_{\|}^{\mu}\right)\right\}\left\{L_{l-1}\left(2 \alpha_{k}\right) L_{n-1}\left(2 \alpha_{k}\right)+L_{l}\left(2 \alpha_{k}\right) L_{n}\left(2 \alpha_{k}\right)\right\} \\
& -\left(m_{N}^{2}-k_{\|}^{2}-k_{\|} \cdot q_{\|}\right) g_{\perp}^{\mu \nu}\left\{L_{l}\left(2 \alpha_{k}\right) L_{n-1}\left(2 \alpha_{k}\right)+L_{l-1}\left(2 \alpha_{k}\right) L_{n}\left(2 \alpha_{k}\right)\right\} \\
& +\kappa_{\rho}\left[\left(q_{\|}^{2} g_{\|}^{\mu \nu}-q_{\|}^{\mu} q_{\|}^{\nu}\right)\left\{L_{l-1}\left(2 \alpha_{k}\right) L_{n-1}\left(2 \alpha_{k}\right)+L_{l}\left(2 \alpha_{k}\right) L_{n}\left(2 \alpha_{k}\right)\right\}\right. \\
& \left.-q_{\|}^{2} g_{\perp}^{\mu \nu}\left\{L_{l}\left(2 \alpha_{k}\right) L_{n-1}\left(2 \alpha_{k}\right)+L_{l-1}\left(2 \alpha_{k}\right) L_{n}\left(2 \alpha_{k}\right)\right\}\right] \\
& +\frac{\kappa_{\rho}^{2}}{4 m_{N}^{2}}\left[8\left\{k_{\perp}^{2}\left(q_{\|}^{2} g^{\mu \nu}-q_{\|}^{\mu} q_{\|}^{\nu}\right)-q_{\|}^{2} g_{\perp}^{\mu \nu}\left\{L_{l}\left(2 \alpha_{k}\right) L_{n-1}\left(2 \alpha_{k}\right)+L_{l-1}\left(2 \alpha_{k}\right) L_{n}\left(2 \alpha_{k}\right)\right\}\right\}\right. \\
& -\left\{2\left(k_{\|} \cdot q_{\|}\right)^{2} g_{\|}^{\mu \nu}+2 q_{\|}^{2} k_{\|}^{\mu} k_{\|}^{\nu}-2\left(k_{\|} \cdot q_{\|}\right)\left(q_{\|}^{\mu} k_{\|}^{\nu}+q_{\|}^{\nu} k_{\|}^{\mu}\right)-\left(m_{N}^{2}+k_{\|}^{2}-k_{\|} \cdot q_{\|}\right)\left(q_{\|}^{2} g_{\|}^{\mu \nu}-q_{\|}^{\mu} q_{\|}^{\nu}\right)\right\} \\
& \times\left\{L_{l-1}\left(2 \alpha_{k}\right) L_{n-1}\left(2 \alpha_{k}\right)+L_{l}\left(2 \alpha_{k}\right) L_{n}\left(2 \alpha_{k}\right)\right\}-\left\{q_{\|}^{2}\left(m_{N}^{2}+k_{\|}^{2}-k_{\|} \cdot q_{\|}\right)-2\left(k_{\|} \cdot q_{\|}\right)^{2}\right\} g_{\perp}^{\mu \nu} \\
& \left.\left.\times\left\{L_{l}\left(2 \alpha_{k}\right) L_{n-1}\left(2 \alpha_{k}\right)+L_{l-1}\left(2 \alpha_{k}\right) L_{n}\left(2 \alpha_{k}\right)\right\}\right]\right] \\
&
\end{aligned}
$$

where the terms involving odd powers of $k_{\perp}^{\mu}$ are discarded as they will vanish while integrating over $d^{2} k_{\perp}$. We now perform the $d^{2} k_{\perp}$ integration using the orthogonality of the Laguerre polynomial (identities provided in Appendix A) to obtain

$$
\begin{aligned}
\tilde{\mathcal{N}}_{\mathrm{p}, n l}^{\mu \nu}\left(q_{\|}, k_{\|}\right)= & -g_{\rho N N}^{2}(-1)^{n+l} \frac{e B}{\pi}\left[4 e B g_{\|}^{\mu \nu} n \delta_{l-1}^{n-1}+\left\{\left(m_{N}^{2}-k_{\|}^{2}-k_{\|} \cdot q_{\|}\right) g_{\|}^{\mu \nu}+2 k_{\|}^{\mu} k_{\|}^{\nu}+\left(q_{\|}^{\mu} k_{\|}^{\nu}+q_{\|}^{\nu} k_{\|}^{\mu}\right)\right\}\left(\delta_{l-1}^{n-1}+\delta_{l}^{n}\right)\right. \\
& -\left(m_{N}^{2}-k_{\|}^{2}-k_{\|} \cdot q_{\|}\right) g_{\perp}^{\mu \nu}\left(\delta_{l-1}^{n}+\delta_{l}^{n-1}\right)+\kappa_{\rho}\left[\left(q_{\|}^{2} g_{\|}^{\mu \nu}-q_{\|}^{\mu} q_{\|}^{\nu}\right)\left(\delta_{l-1}^{n-1}+\delta_{l}^{n}\right)-q_{\|}^{2} g_{\perp}^{\mu \nu}\left(\delta_{l-1}^{n}+\delta_{l}^{n-1}\right)\right] \\
& +\frac{\kappa_{\rho}^{2}}{4 m_{N}^{2}}\left[-4 e B\left(q_{\|}^{2} g_{\|}^{\mu \nu}-q_{\|}^{\mu} q_{\|}^{\nu}\right) n \delta_{l-1}^{n-1}-\left\{2\left(k_{\|} \cdot q_{\|}\right)^{2} g_{\|}^{\mu \nu}+2 q_{\|}^{2} k_{\|}^{\mu} k_{\|}^{\nu}-2\left(k_{\|} \cdot q_{\|}\right)\left(q_{\|}^{\mu} k_{\|}^{\nu}+q_{\|}^{\nu} k_{\|}^{\mu}\right)\right.\right. \\
& \left.\left.\left.-\left(m_{N}^{2}+k_{\|}^{2}-k_{\|} \cdot q_{\|}\right)\left(q_{\|}^{2} g_{\|}^{\mu \nu}-q_{\|}^{\mu} q_{\|}^{\nu}\right)\right\}\left(\delta_{l-1}^{n-1}+\delta_{l}^{n}\right)-\left\{q_{\|}^{2}\left(m_{N}^{2}+k_{\|}^{2}-k_{\|} \cdot q_{\|}\right)-2\left(k_{\|} \cdot q_{\|}\right)^{2}\right\} g_{\perp}^{\mu \nu}\left(\delta_{l-1}^{n}+\delta_{l}^{n-1}\right)\right]\right]
\end{aligned}
$$

It is to be noted that a Kronecker delta with -ve index is zero, which comes from our constraint on the Laguerre polynomials $L_{-1}^{a}=0$.

$$
g_{\mu \nu} \mathcal{N}_{\pi}^{\mu \nu}(q, k)=g_{\rho \pi \pi}^{2}\left[q^{4} k^{\mu} k^{\nu}+(q \cdot k)^{2} q^{2}-q^{2}(q \cdot k) 2 q \cdot k\right]
$$

$$
\mathcal{N}_{\pi}^{00}(q, k)=g_{\rho \pi \pi}^{2}\left[q^{4} k_{0}^{2}+(q \cdot k)^{2} q_{0}^{2}-q^{2}(q \cdot k) 2 q^{0} k^{0}\right]
$$

In this Appendix, we list the explicit forms of $\mathcal{N}^{\mu}{ }_{\mu}$ and $\mathcal{N}^{00}$ for all the different loops. For the zero magnetic field case, we have for the $\pi \pi$ loop and for the NN loop

$$
\begin{aligned}
g_{\mu \nu} \mathcal{N}_{\mathrm{N}}^{\mu \nu}(q, k)= & -8 g_{\rho N N}^{2}\left[\left(m_{N}^{2}-k^{2}-k \cdot q\right) 4+2 k^{2}+q \cdot k+\kappa_{\rho} 3 q^{2}\right. \\
& \left.+\frac{\kappa_{\rho}^{2}}{4 m_{N}^{2}}\left\{\left(m_{N}^{2}+k^{2}-k \cdot q\right) 3 q^{2}-2 q^{2} k^{2}-2(k \cdot q)^{2} 4+4(k \cdot q)^{2}\right\}\right] . \\
\mathcal{N}_{\mathrm{N}}^{00}(q, k)=- & 8 g_{\rho N N}^{2}\left[\left(m_{N}^{2}-k^{2}-k \cdot q\right)+2 k_{0}^{2}+2 q^{0} k^{0}-\kappa_{\rho} \vec{q}^{2}\right. \\
+ & \left.\frac{\kappa_{\rho}^{2}}{4 m_{N}^{2}}\left\{-\left(m_{N}^{2}+k^{2}-k \cdot q\right) \vec{q}^{2}-2 q^{2} k_{0}^{2}-2(k \cdot q)^{2}+4(k \cdot q) q^{0} k^{0}\right\}\right] .
\end{aligned}
$$


The corresponding expressions for the $\pi \pi$ loop for the finite magnetic field case are given by

$$
\begin{aligned}
g_{\mu \nu} \tilde{\mathcal{N}}_{\pi, n l}^{\mu \nu}\left(q_{\|}, k_{\|}\right)= & 4 g_{\rho \pi \pi}^{2}(-1)^{n+l} \frac{e B}{8 \pi}\left[\left\{q_{\|}^{4} k_{\|}^{2}+\left(q_{\|} \cdot k_{\|}\right)^{2} q_{\|}^{2}-q_{\|}^{2}\left(q_{\|} \cdot k_{\|}\right) 2 q_{\|} \cdot k_{\|}\right\} \delta_{l}^{n}\right. \\
& \left.-q_{\|}^{4} \frac{e B}{2}\left\{(2 n+1) \delta_{l}^{n}-(n+1) \delta_{l}^{n+1}-n \delta_{l}^{n-1}\right\}\right] \\
\tilde{\mathcal{N}}_{\pi, n l}^{00}\left(q_{\|}, k_{\|}\right)= & 4 g_{\rho \pi \pi}^{2}(-1)^{n+l} \frac{e B}{8 \pi}\left[q_{\|}^{4} k_{0}^{2}+\left(q_{\|} \cdot k_{\|}\right)^{2} q_{0}^{2}-q_{\|}^{2}\left(q_{\|} \cdot k_{\|}\right) 2 q^{0} k^{0}\right] \delta_{l}^{n},
\end{aligned}
$$

whereas the same for the proton-proton loop are

$$
\begin{aligned}
g_{\mu \nu} \tilde{\mathcal{N}}_{\mathrm{p}, n l}^{\mu \nu}\left(q_{\|}, k_{\|}\right)= & -g_{\rho N N}^{2}(-1)^{n+l} \frac{e B}{\pi}\left[8 e B n \delta_{l-1}^{n-1}+\left\{\left(m_{N}^{2}-k_{\|}^{2}-k_{\|} \cdot q_{\|}\right) 2+2 k_{\|}^{2}+2 q_{\|} \cdot k_{\|}\right\}\left(\delta_{l-1}^{n-1}+\delta_{l}^{n}\right)\right. \\
& -\left(m_{N}^{2}-k_{\|}^{2}-k_{\|} \cdot q_{\|}\right) 2\left(\delta_{l-1}^{n}+\delta_{l}^{n-1}\right)+\kappa_{\rho}\left[q_{\|}^{2}\left(\delta_{l-1}^{n-1}+\delta_{l}^{n}\right)-q_{\|}^{2} 2\left(\delta_{l-1}^{n}+\delta_{l}^{n-1}\right)\right] \\
& +\frac{\kappa_{\rho}^{2}}{4 m_{N}^{2}}\left[-4 e B q_{\|}^{2} n \delta_{l-1}^{n-1}-\left\{2\left(k_{\|} \cdot q_{\|}\right)^{2} 2+2 q_{\|}^{2} k_{\|}^{2}-2\left(k_{\|} \cdot q_{\|}\right) 2 q_{\|} \cdot k_{\|}\right.\right. \\
& \left.\left.\left.-\left(m_{N}^{2}+k_{\|}^{2}-k_{\|} \cdot q_{\|}\right) q_{\|}^{2}\right\}\left(\delta_{l-1}^{n-1}+\delta_{l}^{n}\right)-\left\{q_{\|}^{2}\left(m_{N}^{2}+k_{\|}^{2}-k_{\|} \cdot q_{\|}\right)-2\left(k_{\|} \cdot q_{\|}\right)^{2}\right\} 2\left(\delta_{l-1}^{n}+\delta_{l}^{n-1}\right)\right]\right] \\
\tilde{\mathcal{N}}_{\mathrm{p}, n l}^{00}\left(q_{\|}, k_{\|}\right)= & -g_{\rho N N}^{2}(-1)^{n+l} \frac{e B}{\pi}\left[4 e B n \delta_{l-1}^{n-1}+\left\{\left(m_{N}^{2}-k_{\|}^{2}-k_{\|} \cdot q_{\|}\right)+2 k_{0}^{2}+2 q^{0} k^{0}\right\}\left(\delta_{l-1}^{n-1}+\delta_{l}^{n}\right)\right. \\
& +\kappa_{\rho}\left[-q_{z}^{2}\left(\delta_{l-1}^{n-1}+\delta_{l}^{n}\right)\right]+\frac{\kappa_{\rho}^{2}}{4 m_{N}^{2}}\left[4 e B q_{z}^{2} n \delta_{l-1}^{n-1}-\left\{2\left(k_{\|} \cdot q_{\|}\right)^{2}+2 q_{\|}^{2} k_{0}^{2}-2\left(k_{\|} \cdot q_{\|}\right) 2 q^{0} k^{0}\right.\right. \\
& \left.\left.\left.+\left(m_{N}^{2}+k_{\|}^{2}-k_{\|} \cdot q_{\|}\right) q_{z}^{2}\right\}\left(\delta_{l-1}^{n-1}+\delta_{l}^{n}\right)\right]\right] .
\end{aligned}
$$

[1] V. Skokov, A. Yu. Illarionov, and V. Toneev, Int. J. Mod. Phys. A 24, 5925 (2009).

[2] A. Bzdak and V. Skokov, Phys. Rev. Lett. 110, 192301 (2013).

[3] L. McLerran and V. Skokov, Nucl. Phys. A929, 184 (2014).

[4] K. Tuchin, Phys. Rev. C 88, 024910 (2013).

[5] K. Tuchin, Phys. Rev. C 87, 024912 (2013).

[6] K. Tuchin, Adv. High Energy Phys. 2013, 490495 (2013).

[7] T. Vachaspati, Phys. Lett. B 265, 258 (1991).

[8] D. Grasso and H. R. Rubinstein, Phys. Rep. 348, 163 (2001).

[9] M. Giovannini, Int. J. Mod. Phys. D 13, 391 (2004).

[10] M. Giovannini, Classical Quantum Gravity 35, 084003 (2018).

[11] V. M. Kaspi and A. M. Beloborodov, Annu. Rev. Astron. Astrophys. 55, 261 (2017).

[12] E. J. Ferrer, V. de la Incera, and C. Manuel, Phys. Rev. Lett. 95, 152002 (2005).

[13] E. J. Ferrer, V. de la Incera, and C. Manuel, Nucl. Phys. B747, 88 (2006).
[14] E. J. Ferrer and V. de la Incera, Phys. Rev. D 76, 045011 (2007).

[15] K. Fukushima and H. J. Warringa, Phys. Rev. Lett. 100, 032007 (2008).

[16] B. Feng, D. Hou, H.-c. Ren, and P.-p. Wu, Phys. Rev. Lett. 105, 042001 (2010).

[17] S. Fayazbakhsh and N. Sadooghi, Phys. Rev. D 82, 045010 (2010).

[18] S. Fayazbakhsh and N. Sadooghi, Phys. Rev. D 83, 025026 (2011).

[19] M. N. Chernodub, Phys. Rev. D 82, 085011 (2010).

[20] M. N. Chernodub, Lect. Notes Phys. 871, 143 (2013).

[21] C. Vafa and E. Witten, Nucl. Phys. B234, 173 (1984).

[22] M. N. Chernodub, Phys. Rev. D 86, 107703 (2012).

[23] C. Li and Q. Wang, Phys. Lett. B 721, 141 (2013).

[24] Y. Hidaka and A. Yamamoto, Phys. Rev. D 87, 094502 (2013).

[25] H. Liu, L. Yu, and M. Huang, Phys. Rev. D 91, 014017 (2015).

[26] M. Kawaguchi and S. Matsuzaki, Phys. Rev. D 93, 125027 (2016). 
[27] H. Liu, L. Yu, and M. Huang, Chin. Phys. C 40, 023102 (2016).

[28] S. Ghosh, A. Mukherjee, M. Mandal, S. Sarkar, and P. Roy, Phys. Rev. D 94, 094043 (2016).

[29] A. Bandyopadhyay and S. Mallik, Eur. Phys. J. C 77, 771 (2017).

[30] M. Kawaguchi and S. Matsuzaki, Eur. Phys. J. A 53, 68 (2017).

[31] S. Ghosh, A. Mukherjee, M. Mandal, S. Sarkar, and P. Roy, Phys. Rev. D 96, 116020 (2017).

[32] E. Elizalde, S. D. Odintsov, A. Romeo, A. A. Bytsenko, and S. Zerbini, Zeta Regularization Techniques with Applications (World Scientific, Singapore, 1994).

[33] O. Krehl, C. Hanhart, S. Krewald, and J. Speth, Phys. Rev. C 62, 025207 (2000).

[34] M. L. Bellac, Thermal Field Theory, Cambridge Monographs on Mathematical Physics (Cambridge University Press, Cambridge, England, 2011).

[35] S. Mallik and S. Sarkar, Hadrons at Finite Temperature (Cambridge University Press, Cambridge, England, 2016).

[36] J. S. Schwinger, Phys. Rev. 82, 664 (1951).

[37] A. Ayala, A. Sanchez, G. Piccinelli, and S. Sahu, Phys. Rev. D 71, 023004 (2005).

[38] S. Ghosh and V. Chandra, Phys. Rev. D 98, 076006 (2018).

[39] P. Chakraborty, arXiv:1711.04404.

[40] A. N. Gorban, Model Reduction and Coarse-Graining Approaches for Multiscale Phenomena (Springer, Berlin Heidelberg, 2006), Vol. 117.

[41] T. E.-A. P. Ehrenfest, The Conceptual Foundations of the Statistical Approach in Mechanics (Dover, Phoenix, 2002).

[42] M. Dey, V. L. Eletsky, and B. L. Ioffe, Phys. Lett. B 252, 620 (1990).
[43] J. I. Kapusta and E. V. Shuryak, Phys. Rev. D 49, 4694 (1994).

[44] P. M. Hohler and R. Rapp, Phys. Lett. B 731, 103 (2014).

[45] G. E. Brown and M. Rho, Phys. Rev. Lett. 66, 2720 (1991).

[46] J. Alam, S. Sarkar, P. Roy, T. Hatsuda, and B. Sinha, Ann. Phys. (N.Y.) 286, 159 (2000).

[47] S. Leupold, V. Metag, and U. Mosel, Int. J. Mod. Phys. E 19, 147 (2010).

[48] V. Voronyuk, V. D. Toneev, W. Cassing, E. L. Bratkovskaya, V. P. Konchakovski, and S. A. Voloshin, Phys. Rev. C 83, 054911 (2011).

[49] K. Tuchin, Phys. Rev. C 82, 034904 (2010).

[50] K. Tuchin, Phys. Rev. C 83, 017901 (2011).

[51] K. Tuchin, Phys. Rev. C 93, 014905 (2016).

[52] V. Roy, S. Pu, L. Rezzolla, and D. H. Rischke, Phys. Rev. C 96, 054909 (2017).

[53] A. Das, S. S. Dave, P. S. Saumia, and A. M. Srivastava, Phys. Rev. C 96, 034902 (2017).

[54] G. Inghirami, L. Del Zanna, A. Beraudo, M. H. Moghaddam, F. Becattini, and M. Bleicher, Eur. Phys. J. C 76, 659 (2016).

[55] P. Filip, J. Phys. Conf. Ser. 636, 012013 (2015).

[56] D. Albuquerque, Nucl. Phys. A982, 823 (2019); Proceedings of the 27th International Conference on Ultrarelativistic Nucleus-Nucleus Collisions: Quark Matter, 2018.

[57] S. Acharya et al. (ALICE Collaboration), arXiv:1805.04365.

[58] Y. F. Yuan and J. L. Zhang, Astrophys. J. 525, 950 (1999).

[59] F. X. Wei, G. J. Mao, C. M. Ko, L. S. Kisslinger, H. Stoecker, and W. Greiner, J. Phys. G 32, 47 (2006).

[60] M. E. Peskin and D. V. Schroeder, An Introduction to Quantum Field Theory (Addison-Wesley, Reading, MA, 1995). 